$\boldsymbol{u}^{b}$

$b$ UNIVERSITÄT

BERN

Faculty of Business, Economics and Social Sciences

Department of Social Sciences

University of Bern Social Sciences Working Paper No. 37

\title{
Relative distribution analysis in Stata
}

Ben Jann

Current version: June 21, 2021

First version: $\quad$ September 18, 2020

http://ideas.repec.org/p/bss/wpaper/37.html

http://econpapers.repec.org/paper/bsswpaper/37.htm 


\title{
Relative distribution analysis in Stata
}

\author{
Ben Jann \\ Institute of Sociology \\ University of Bern \\ ben.jann@soz. unibe.ch
}

June 21, 2021

\begin{abstract}
In this paper I discuss the method of relative distribution analysis and present Stata software implementing various elements of the methodology. The relative distribution is the distribution of the relative ranks that the outcomes from one distribution take on in another distribution. The methodology can be used, for example, to compare the distribution of wages between men and women. Another example would be the analysis of changes in the distribution of earnings over time. Of interest are the relative cumulative distribution (relative CDF), the relative density (relative PDF), as well as summary measures such as the median relative polarization (MRP). The presented software can be used to estimate these quantities and also provides functionality such as locationand-shape decompositions or covariate balancing. Statistical inference is implemented in terms of influence functions and supports estimation for complex samples.
\end{abstract}

Keywords: Stata, reldist, relative distribution, relative density, median relative polarization, divergence, Gastwirth index, reweighting, influence function 


\section{Contents}

1 Introduction $\quad 4$

2 Theory $\quad 5$

2.1 Distribution function and density . . . . . . . . . . . . . . . 5

2.2 Relative ranks . . . . . . . . . . . . . . . . . . 6

2.3 The relative distribution function . . . . . . . . . . . . . . . . 6

2.4 The relative density function . . . . . . . . . . . . . . . . . . . . . 8

2.5 Location and shape decomposition . . . . . . . . . . . . . . 8

2.6 Summary measures . . . . . . . . . . . . . . . . . . . 10

2.6.1 Divergence . . . . . . . . . . . . . . . . . 10

2.6.2 Polarization ...................... 11

2.6.3 Other summary measures . . . . . . . . . . . . . . . . . 12

2.7 Covariate balancing . . . . . . . . . . . . . . . . . 12

2.7.1 Integrating over conditional distributions . . . . . . . . . . . . . . . . 12

2.7.2 Reweighting . . . . . . . . . . . . . . . . . 13

3 Estimation $\quad 14$

3.1 The relative distribution function . . . . . . . . . . . . . . . . . 15

3.2 Computing relative ranks . . . . . . . . . . . . . . . . . . . . . . . . 16

3.3 The relative density function . . . . . . . . . . . . . . . . . 18

3.3.1 Kernel density estimation for continuous data . . . . . . . . . . 18

3.3.2 Histogram density estimation . . . . . . . . . . . . . . . . . . . 19

3.3.3 Discrete relative density for categorical data . . . . . . . . . . . . . 20

3.4 Divergence . . . . . . . . . . . . . . . . . . . 20

3.4.1 Continuous data . . . . . . . . . . . . . . . . 20

3.4 .2 Categorical data . . . . . . . . . . . . . . . . . . . 21

3.5 Median relative polarization . . . . . . . . . . . . . . . . . . . 21

3.6 Covariate balancing . . . . . . . . . . . . . . . . . . . . 22

3.7 Standard errors . . . . . . . . . . . . . . . . . . . . . . . . 22

3.7.1 Variance estimation by means of influence functions . . . . . . . . . . 23

3.7.2 Influence function for the relative CDF . . . . . . . . . . . . . . 26

3.7.3 Influence function for the relative histogram . . . . . . . . . . . . . . 29

3.7.4 Influence function for the relative PDF . . . . . . . . . . . . . . . . . 29

3.7.5 Influence function for the discrete relative density . . . . . . . . . . . 31

3.7.6 Influence functions for divergence measures . . . . . . . . . . . . . . . 32 
3.7.7 Influence functions for polarization indices . . . . . . . . . . . 32

3.7 .8 Influence functions for descriptive statistics . . . . . . . . . . . 33

3.7.9 Influence functions in case of covariate balancing . . . . . . . . . . . 34

4 The reldist command $\quad 36$

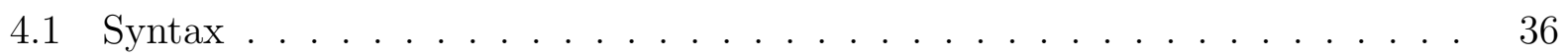

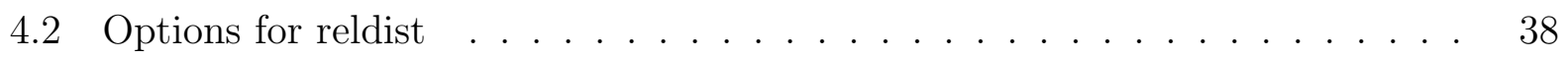

4.3 Options for reldist graph . . . . . . . . . . . . . . . . . 46

4.4 Saved results . . . . . . . . . . . . . . . . . . . . . . . . 49

5 Examples $\quad 50$

5.1 Wage mobility in two eras . . . . . . . . . . . . . . . 50

5.2 Processing results from reldist . . . . . . . . . . . . . . . 63

5.3 Survey estimation . . . . . . . . . . . . . . . . 67

$\begin{array}{lll}6 & \text { Acknowledgements } & 68\end{array}$ 


\section{Introduction}

Although earlier work on relative distributions and related approaches can be found in the statistical literature (e.g., Ćwik and Mielniczuk, 1989, 1993), the methodology has not been popular in applied work before Mark S. Handcock, Martina Morris, and coauthors introduced it to the social sciences in some influential applied (Morris et al., 1994; Bernhardt et al., 1995, 2001) and methodological contributions (Handcock and Morris, 1998, 1999; Handcock and Janssen, 2002) in the mid 1990s and early 2000s. Even today, however, relative distribution methods do not seem to experience very widespread use, which might, in part, be due to lack of user-friendly statistical software supporting such analyses (apart from an $\mathrm{R}$ package by Handcock and Aldrich, 2002; see Handcock, 2016).

Nevertheless, I believe that relative distribution analysis is a valuable complement to other approaches for distributional comparisons, which typically look at differences in (counterfactual) density, distribution, or quantile functions (e.g., DiNardo et al., 1996, Chernozhukov et al., 2013). A key feature of relative distribution analysis is that it focuses on positions within distributions rather than on absolute outcome values. The methodology can be used, for example, to study how wage distributions differ by gender or ethnic groups, or how income polarization changed over time. A few examples from the literature illustrate the scope of potential applications: Alderson et al. (2005) studied changes in income inequality in several countries; Bliege Bird et al. (2008) analyzed the anthropogenic influence on vegetational diversity in Australia; Del Giudice (2011) looked at gender differences in adult romantic attachment; Eggers and Spirling (2016) studied cohesive party voting in the British House of Commons between 1836 and 1910; Clementi et al. (2018) analyzed changes in the consumption distribution over time in Ghana; and Panek and Zwierzchowski (2020) studied changes in household income polarization in Poland.

In an attempt to improve the accessibility of the methodology to applied researchers, I provide an overview of relative distribution methods in this article and I present software that makes the methodology available in Stata. The software - called reldist - can be used to estimate and plot the relative density function (relative PDF), a histogram of the relative distribution, or the relative distribution function (relative CDF). Furthermore, it computes relative polarization indices, distributional divergence measures, as well as descriptive statistics of the relative data, and it supports the decomposition of the relative distribution by adjusting for location, scale, and shape differences, or for differences in covariate distributions. Estimation of standard errors and confidence intervals is provided for all quantities, including support for complex samples. I tried to make the software as versatile as possible, while at the same time maintaining user friendliness, for example, by following official Stata's standards in terms of syntax, output, and stored results.

The article is structured as follows. In the next section I give an overview of the main concepts of relative distribution analysis, including definitions of relative ranks and the relative distribution as well as elements such as location and shape decompositions, distributional divergence and relative polarization summary measures, and covariate adjustment approaches. 
Most of the discussed material is also covered in Handcock and Morris (1999), but I focus on elements I consider most relevant from an applied perspective and I use a somewhat different notation. Furthermore, I introduce reweighting as an additional strategy for covariate adjustment. In section 3 , I then discuss the computational details involved in the estimation of the quantities presented in section 2. Different variants of how to compute relative ranks, the relative cumulative distribution, the relative density, the relative histogram, summary measures, and covariate balancing are covered, also making a distinction between a continuous and categorical outcomes when relevant. Again, many of the relevant issues are also addressed by Handcock and Morris (1999), but my exposition is more focused on specific implementation. Furthermore, I cover the estimation of sampling variances by means of influence functions. Section 4 then introduces the software and its options and section 5 provides a number of worked examples.

\section{Theory}

In this section I summarize the main statistical concepts that are relevant for relative distribution analysis. For an in-depth treatment of the topic see Handcock and Morris (1999). For a more recent introduction also see Chapter 5 in Hao and Naiman (2010).

\subsection{Distribution function and density}

Let $Y$ be a continuous outcome variable of interest. $Y$ is assumed a random variable with distribution function

$$
F_{Y}(y)=P(Y \leq y), \quad y \in \mathbb{R}
$$

That is, for any value $y$, the distribution function provides the probability of $Y$ taking on a value that is smaller than or equal to $y$. The density function of $Y$ is then defined as the first derivative of the distribution function, that is,

$$
f_{Y}(y)=F_{Y}^{\prime}(y)=\frac{\mathrm{d} F_{Y}(y)}{\mathrm{d} y}
$$

Hence, the integral of the density from $-\infty$ to $y$ is equal to the value of the distribution function at value $y$ :

$$
F_{Y}(y)=\int_{-\infty}^{y} f_{Y}(t) \mathrm{d} t
$$

Likewise, the integral of the density between values $a$ and $b$ provides the probability that $Y$ falls into interval $(a, b]$ :

$$
P(a<Y \leq b)=F_{Y}(b)-F_{Y}(a)=\int_{a}^{b} f_{Y}(y) \mathrm{d} y
$$


Finally, let $q_{Y}(p)=F_{Y}^{-1}(p)$ be the inverse of $F_{Y}$, that is, the quantile function of $Y$, such that

$$
y=q_{Y}\left(F_{Y}(y)\right)=F_{Y}^{-1}\left(F_{Y}(y)\right)
$$

\subsection{Relative ranks}

Define

$$
r_{Y}(y)=F_{Y}(y)
$$

as the "relative rank" of outcome $y$ in distribution $F_{Y}$. Because $F_{Y}$ is a distribution function, $r$ lies between 0 and 1 . Handcock and Morris (1999) call $r$ the "relative data", Ćwik and Mielniczuk (1989) speak of the "grade transformation".

Relative ranks have a distribution themselves which depends on the distribution of the $y$ values at which $r_{Y}(y)$ is evaluated. For example, if the $y$ values are distributed according to $F_{Y}$, then $r$ has an uniform distribution.

\subsection{The relative distribution function}

Let $F_{X}$ be a comparison distribution and $F_{Y}$ be a reference distribution. In relative distribution analysis we are interested in how $F_{X}$ is distributed relative to $F_{Y}$. The relative distribution function (relative $\mathrm{CDF}$ ) of $F_{X}$ with respect to $F_{Y}$ is defined as the distribution of the relative ranks that outcome values distributed according to $F_{X}$ take on in distribution $F_{Y}$. That is, we are interested the distribution of $r_{Y}(y)$ for $y$ values distributed according to $F_{X}$, which can be obtained by inverting $r$ to $y$ using $F_{Y}^{-1}$ and then applying $F_{X}$. Hence, the relative $\mathrm{CDF}$ is given as

$$
G(r)=F_{X}\left(F_{Y}^{-1}(r)\right), \quad r \in[0,1]
$$

Stated differently, for each value of $r=F_{Y}(y)$ the relative CDF obtains the corresponding value of $F_{X}(y)$, keeping $y$ fixed, which leads to the tuples

$$
\left(F_{X}(y), F_{Y}(y)\right), \quad y \in \mathbb{R}
$$

Plotted in a diagram with $r\left(=F_{Y}(y)\right)$ on the horizontal axis and $G(r)\left(=F_{X}(y)\right)$ on the vertical axis, all points will lie on the diagonal if the two distributions are identical (that is, $G(r)=r$ in this case, as can easily be seen in equation 7). ${ }^{1}$ If the outcome values in the comparison distribution tend to be lower than the outcome values in the reference distribution, the points will lie above the diagonal (and vice versa). The relative distribution might also cross the diagonal, for example, if one of the distributions is more polarized than the other. Figure 1 provides an illustration. On the left, three examples of the density functions of two distributions are shown. In the middle panel, the corresponding relative distribution functions are displayed.

\footnotetext{
${ }^{1}$ The diagram of $F_{X}$ by $F_{Y}$ is also known as "probability-probability plot" (P-P plot; for a Stata implementation see Cox, 2004).
} 

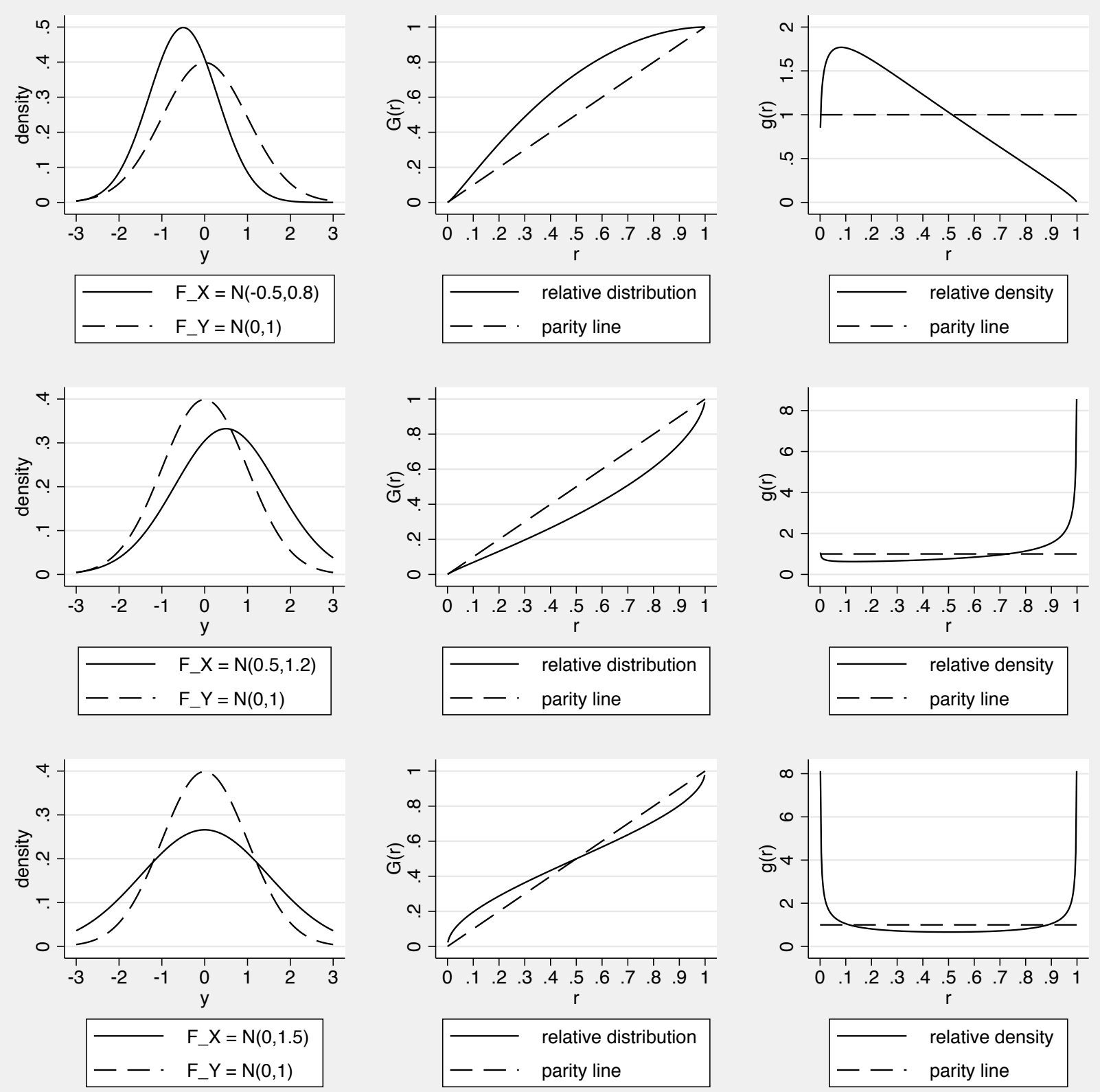

Figure 1: Illustration of the relative distribution 


\subsection{The relative density function}

Since $G(r)$ is a distribution function, we can take the first derivative to obtain the density. Employing the chain rule, the relative density function (relative PDF) of $F_{X}$ with respect to $F_{Y}$ can be written as

$$
g(r)=\frac{\mathrm{d} G(r)}{\mathrm{d} r}=\frac{f_{X}\left(F_{Y}^{-1}(r)\right)}{f_{Y}\left(F_{Y}^{-1}(r)\right)}, \quad r \in[0,1]
$$

As can be seen, the relative density is equal to the ratio of the densities of the two distributions at a specific $y$ value (i.e. $g(r)$ is equal to the ratio of the two densities at the $y$ value equal to quantile $r$ of $F_{Y}$ ). Nonetheless, $g(r)$ is a proper density function as it is positive and integrates to 1 .

If the two compared distributions are identical, $g(r)$ will be equal to 1 for all $r$, as is easy to see in (9). If the comparison distribution tends to have lower values than the reference distribution, the relative density will be larger than 1 at low values of $r$ and smaller than 1 for large $r$ (and vice versa). Likewise, assuming similar locations of the two distribution, if the comparison distribution is more polarized than the reference distribution, the relative density will be larger than 1 at small and large values of $r$, and below 1 in between (and vice versa). An illustration of different situations is provided in the right panel of Figure 1.

\subsection{Location and shape decomposition}

Distributions can have different "locations", meaning that they differ, say, in their mean or median. If a large location difference exist, the relative CDF and PDF will be dominated by this difference. In many application it may thus be informative to distinguish between a "location effect" and the difference in distributional shape, net of location.

As shown by Handcock and Morris (1999), the overall relative density can be decomposed into a "location effect" and a "shape effect" by constructing a location-adjusted distribution and then using this counterfactual distribution in place of either $F_{X}$ or $F_{Y}$. For example, let

$$
\widetilde{Y}=Y-\mu_{Y}+\mu_{X}
$$

be a location-adjusted variant of $Y$, where $\mu$ is a location measure such as the median or the mean. In general, if $\widetilde{Y}=t(Y)$, the distribution of $\widetilde{Y}$ is equal to $F_{Y}\left(t^{-1}(y)\right)$. This means that

$$
F_{\widetilde{Y}}(y)=P\left(Y-\mu_{Y}+\mu_{X} \leq y\right)=P\left(Y \leq y+\mu_{Y}-\mu_{X}\right)=F_{Y}\left(y+\mu_{Y}-\mu_{X}\right)
$$

is a location-adjusted reference distribution that has the same location as the comparison distribution. The overall relative density can then be written as

$$
g(r)=\frac{f_{X}\left(F_{Y}^{-1}(r)\right)}{f_{Y}\left(F_{Y}^{-1}(r)\right)}=\underbrace{\frac{f_{\widetilde{Y}}\left(F_{Y}^{-1}(r)\right)}{f_{Y}\left(F_{Y}^{-1}(r)\right)}}_{\text {location effect }} \times \underbrace{\frac{f_{X}\left(F_{Y}^{-1}(r)\right)}{f_{\widetilde{Y}}\left(F_{Y}^{-1}(r)\right)}}_{\text {shape effect }}
$$


The first factor, the location effect, is equal to the ratio between the density of the locationadjusted reference distribution and the unadjusted reference distribution, the second factor, the shape effect, is the ratio between the density of the (unadjusted) comparison distribution and the location-adjusted reference distribution. However, note that

$$
\frac{f_{X}\left(F_{Y}^{-1}(r)\right)}{f_{\widetilde{Y}}\left(F_{Y}^{-1}(r)\right)}, \quad r \in[0,1]
$$

is not a proper density because it is evaluated over $y$ values distributed according to $F_{Y}$ instead of $F_{\tilde{Y}}$. It may therefore be more useful to characterize the shape effect by the adjusted relative PDF

$$
g_{X \widetilde{Y}}(r)=\frac{f_{X}\left(F_{\widetilde{Y}}^{-1}(r)\right)}{f_{\widetilde{Y}}\left(F_{\widetilde{Y}}^{-1}(r)\right)}
$$

or the corresponding adjusted relative $\mathrm{CDF}$

$$
G_{X \widetilde{Y}}(r)=F_{X}\left(F_{\widetilde{Y}}^{-1}(r)\right)
$$

Instead of adjusting $F_{Y}$, the decomposition could also be defined by adjusting the comparison distribution. That is, we could use

$$
\widetilde{X}=X-\mu_{X}+\mu_{Y} \quad \text { with } \quad F_{\widetilde{X}}(y)=F_{X}\left(y+\mu_{X}-\mu_{Y}\right)
$$

such that

$$
g(r)=\frac{f_{X}\left(F_{Y}^{-1}(r)\right)}{f_{Y}\left(F_{Y}^{-1}(r)\right)}=\underbrace{\frac{f_{X}\left(F_{Y}^{-1}(r)\right)}{f_{\widetilde{X}}\left(F_{Y}^{-1}(r)\right)}}_{\text {location effect }} \times \underbrace{\frac{f_{\widetilde{X}}\left(F_{Y}^{-1}(r)\right)}{f_{Y}\left(F_{Y}^{-1}(r)\right)}}_{\text {shape effect }}
$$

Similar as above, one of the components is not a proper density. To describe the location effect we may thus prefer

$$
g_{X \widetilde{X}}(r)=\frac{f_{X}\left(F_{\tilde{X}}^{-1}(r)\right)}{f_{\widetilde{X}}\left(F_{\tilde{X}}^{-1}(r)\right)} \quad \text { and } \quad G_{X \widetilde{X}}(r)=F_{X}\left(F_{\widetilde{X}}^{-1}(r)\right)
$$

instead of $f_{X}\left(F_{Y}^{-1}(r)\right) / f_{\widetilde{X}}\left(F_{Y}^{-1}(r)\right)$. Results from (12) and (17) will generally not be the same, although for some of the measures discussed below it does not matter whether we adjust $F_{X}$ or $F_{Y}$.

So far, an additive location shift has been used to adjust the comparison or reference distribution. For variables that can only be positive (e.g. wages) it may be more natural to use a multiplicative shift and, hence, rescale the data proportionally. A multiplicative location adjustment of the reference distribution is given by $\widetilde{Y}=Y \cdot \mu_{X} / \mu_{Y}$ and hence

$$
F_{\widetilde{Y}}(y)=F_{Y}\left(y \cdot \mu_{Y} / \mu_{X}\right)
$$


The comparison distribution could be adjusted analogously. Furthermore, besides the location, we could also adjust the scale of the distributions. An (additive) location and scale adjustment of the reference distribution could be accomplished using $\widetilde{Y}=\left(Y-\mu_{Y}\right) \cdot s_{X} / s_{Y}+\mu_{X}$ such that

$$
F_{\widetilde{Y}}(y)=F_{Y}\left(\left(y-\mu_{X}\right) \cdot s_{Y} / s_{X}+\mu_{Y}\right)
$$

where $s$ is a scale measure such as the IQR (interquartile range) or the standard deviation. For the multiplicative adjustment there is no natural way to take account of the scale. However, we can implement a proportional location and scale adjustment on the logarithmic scale as $\widetilde{Y}=\exp \left(\left(\ln (Y)-\mu_{\ln (Y)}\right) \cdot s_{\ln (X)} / s_{\ln (Y)}+\mu_{\ln (X)}\right)$ such that

$$
F_{\widetilde{Y}}(y)=F_{Y}\left(\exp \left(\left(\ln (y)-\mu_{\ln (X)}\right) \cdot s_{\ln (Y)} / s_{\ln (X)}+\mu_{\ln (Y)}\right)\right)
$$

\subsection{Summary measures}

\subsubsection{Divergence}

Handcock and Morris (1999) suggest the Pearson chi-squared divergence and the KullbackLeibler divergence (relative entropy) as measures for distributional divergence, that is, as summary measures for the overall difference between the comparison distribution and the reference distribution. The chi-squared divergence between $F_{X}$ and $F_{Y}$ is defined as

$$
\chi^{2}=\int_{-\infty}^{\infty} \frac{\left(f_{X}(y)-f_{Y}(y)\right)^{2}}{f_{Y}(y)} \mathrm{d} y=\int_{0}^{1}(g(r)-1)^{2} \mathrm{~d} r
$$

The equality between the first and second expression follows from the substitution rule for integrals, noting that $y=F_{Y}^{-1}(r)$ and $\mathrm{d} F_{Y}^{-1}(r) / \mathrm{d} r=1 / f_{Y}\left(F_{Y}^{-1}(r)\right)$. Likewise, the KullbackLeibler divergence, which has an information-theoretic interpretation (negative entropy of the relative density), is defined as

$$
\mathrm{KL}=\int_{-\infty}^{\infty} \ln \left(\frac{f_{X}(y)}{f_{Y}(y)}\right) f_{X}(y) \mathrm{d} y=\int_{0}^{1} \ln (g(r)) g(r) \mathrm{d} r
$$

For both measures, the divergence of $F_{X}$ with respect to $F_{Y}$ is not, in general, equal to the divergence of $F_{Y}$ with respect to $F_{X}$. That is, the direction from which we look at the problem matters. An example for a symmetric divergence measure ${ }^{2}$ is the total variation distance

$$
\mathrm{TVD}=\int_{-\infty}^{\infty} \frac{1}{2}\left|\frac{f_{X}(y)}{f_{Y}(y)}-1\right| f_{Y}(y) \mathrm{d} y=\int_{0}^{1} \frac{1}{2}|g(r)-1| \mathrm{d} r
$$

which is equal to half the area between the relative density curve and the parity line. Besides being symmetric, the TVD has an intuitive interpretation: it quantifies the proportion of data

\footnotetext{
${ }^{2}$ That is, the comparison and reference distribution can be swapped without changing the measure. The equality holds in theory; in an empirical application the agreement will only be approximate due to the smoothing involved in density estimation.
} 
mass that would have to be redistributed in one of the distributions to make it equal to the other distribution. In case of categorical data the total variation distance is equal to the dissimilarity index by Duncan and Davis (1953), which is often used in analyses of segregation (for Stata implementations see, e.g., Jann, 2004 or Reardon and Townsend, 1999).

For all three measures, in a location and shape decomposition, the location-effect divergence and the shape-effect divergence do not add up to the overall divergence. For example, we could location-adjust the reference distribution as in (10) and then obtain the locationeffect divergence from $g_{\widetilde{Y} Y}(r)$ and the shape-effect divergence from $g_{X \widetilde{Y}}(r)$. Unfortunately these two divergences do not add up to the overall divergence. For the Kullback-Leibler divergence, however, as pointed out by Handcock and Morris (1999), the following equality holds

$$
\mathrm{KL}=\mathrm{KL}_{X \tilde{Y} Y}+\mathrm{KL}_{X \widetilde{Y}}
$$

where $\mathrm{KL}_{X \widetilde{Y} Y}$ is a (negative) cross entropy defined as

$$
\mathrm{KL}_{X \widetilde{Y} Y}=\int_{-\infty}^{\infty} \ln \left(\frac{f_{\widetilde{Y}}(y)}{f_{Y}(y)}\right) f_{X}(y) \mathrm{d} y=\int_{0}^{1} \ln \left(g_{\widetilde{Y} Y}(r)\right) g(r) \mathrm{d} r
$$

This suggest that, in practice, it may make sense to identify the location-effect divergence as the difference between the overall divergence and the shape-effect divergence. An advantage of such an approach is also that results will not depend on whether we adjust the reference distribution or the comparison distribution.

\subsubsection{Polarization}

To compare the degree of inequality between the comparison distribution and the reference distribution, Handcock and Morris (1999) suggest the median relative polarization index (MRP). The index is positive if the comparison distribution is more unequal than the reference distribution; if the reference distribution is more unequal than the comparison distribution, the index will be negative. The MRP is defined as

$$
\operatorname{MRP}=4 \cdot E_{X}\left(\left|r_{\widetilde{Y}}(y)-0.5\right|\right)-1 \quad \operatorname{MRP} \in[-1,1]
$$

where $E_{X}$ is the expectation over the comparison distribution and $r_{\widetilde{Y}}(y)$ is the relative rank of $y$ in the location-adjusted reference distribution (using the median as location measure). The justification for the MRP is that the median of the location-adjusted relative ranks is 0.5 and that the location-adjusted relative ranks will have a uniform distribution if the two distributions have the same shape. In this case, $E_{X}\left(\left|r_{\widetilde{Y}}(y)-0.5\right|\right)$ is equal to $1 / 4$, such that MRP is 0 . In the extreme case that all data mass of the comparison distribution is located in regions below and above the range of the location-adjusted reference distribution, $r_{\widetilde{Y}}(y)$ will be zero or one for all $y$ with positive density in $F_{X}$, such that $E_{X}\left(\left|r_{\widetilde{Y}}(y)-0.5\right|\right)=.5$ and, hence, $\mathrm{MRP}=1$. In the opposite extreme, $r_{\widetilde{Y}}(y)$ will always be .5 , leading to an MRP of -1 . 
The MRP can be decomposed into a lower (LRP) and upper polarization index (URP) that quantify the relative polarization in the lower or upper half of the distribution, respectively:

$$
\begin{aligned}
\mathrm{LRP} & =4 \cdot E_{X}\left(\operatorname{abs}\left(r_{\widetilde{Y}}(y)-0.5\right) \mid r_{\widetilde{Y}}(y) \leq 0.5\right)-1 \\
\mathrm{URP} & =4 \cdot E_{X}\left(\operatorname{abs}\left(r_{\widetilde{Y}}(y)-0.5\right) \mid r_{\widetilde{Y}}(y)>0.5\right)-1
\end{aligned}
$$

Since the conditional expectations in the definitions of LRP and URP each cover half of the distribution of the location-adjusted relative ranks, the total polarization index is equal to the average of the lower and upper indices, that is

$$
\mathrm{MRP}=0.5 \cdot \mathrm{LRP}+0.5 \cdot \mathrm{URP}
$$

\subsubsection{Other summary measures}

Descriptive statistics of the relative ranks comprise a further class of relative distribution summary measures. Quantities of interested may be, for example, the mean or median of the relative ranks, or their standard deviation or interquartile range.

Note that the mean of the relative ranks is equivalent to the Gastwirth index, which measures the "probability that a randomly selected woman earns at least as much as a randomly chosen man" (Gastwirth, 1975, 33). ${ }^{3}$

\subsection{Covariate balancing}

\subsubsection{Integrating over conditional distributions}

Handcock and Morris (1999) discuss covariate adjustment in terms of conditional distributions integrated over covariates. I will slightly change notation for the following exposition. Let $D \in\{0,1\}$ be an indicator distinguishing between a comparison group $(D=1)$ and a reference group $(D=0)$ and let $Y$ be an outcome variable available in both groups. The comparison distribution is $F_{Y \mid D=1}$, that is, the distribution of $Y$ in group $D=1$; the reference distribution is $F_{Y \mid D=0}$. Furthermore, let $Z$ be a continuous covariate. Our goal is to obtain the relative distribution of $F_{Y \mid D=1}$ with respect to $F_{Y \mid D=0}$ while adjusting for possible differences in the distribution of $Z$ between the two groups.

The marginal distribution of $Y$ in group $d$ can be written as

$$
F_{Y \mid D=d}(y)=\int_{-\infty}^{\infty} f_{Z \mid D=d}(z) F_{Y \mid D=d, Z}(y \mid z) \mathrm{d} z
$$

\footnotetext{
${ }^{3}$ To be precise, according to the formal definition given by Gastwirth (1975), the index is equal to one minus the average relative rank of men's earnings in the distribution of women's earnings. However, due to the symmetry of the problem, this is equivalent to the average relative rank of women's earnings in the distribution of men's earnings. For a discussion of the Gastwirth index also see Le Breton et al. (2012).
} 
where $f_{Z}(z)$ is the density of $Z$ and $F_{Y \mid Z}(y \mid z)$ is the conditional distribution of $Y$ given $Z$. A counterfactual distribution can now be constructed by replacing one of the components. For example,

$$
F_{Y \mid D=0}^{C}(y)=\int_{-\infty}^{\infty} f_{Z \mid D=1}(z) F_{Y \mid D=0, Z}(y \mid z) \mathrm{d} z
$$

is the marginal distribution of $Y$ that we would expect in the reference group if it had the same covariate distribution as the comparison group. That is, we can obtain the counterfactual distribution by integrating the conditional distribution of $Y$ in the reference group over the covariate distribution of the comparison group. The covariate-adjusted relative distribution can then be obtained by comparing $F_{Y \mid D=1}$ with $F_{Y \mid D=0}^{C} \cdot{ }^{4}$

The approach can be generalized to multiple covariates by integrating over the joint distribution of all covariates or to discrete covariates by taking weighted sums instead of integrals.

\subsubsection{Reweighting}

An equivalent but more attactive approach from an applied perspective is to conceptualize covariate-adjustment as reweighting in the spirit of DiNardo et al. (1996). Define

$$
P(D=1 \mid Z=z)=1-P(D=0 \mid Z=z)
$$

as the conditional probability of belonging to the comparison group given $Z$, where $Z$ is a vector of covariates. Furthermore, define

$$
\Psi(z)=\frac{P(D=1 \mid Z=z) / P(D=1)}{P(D=0 \mid Z=z) / P(D=0)}
$$

We can then write the counterfactual distribution of $Y$ in the reference group as

$$
F_{Y \mid D=0}^{C}(y)=\int_{-\infty}^{\infty} f_{Z \mid D=0}(z) F_{Y \mid D=0, Z}(y \mid z) \Psi(z) \mathrm{d} z
$$

This indicates that the counterfactual distribution can be estimated by simply reweighting the data by an estimate of $\Psi(z) .{ }^{5}$ Mathematically, (34) is equivalent to (31) because

$$
\Psi(z)=\frac{P(D=1 \mid Z=z) / P(D=1)}{P(D=0 \mid Z=z) / P(D=0)}=\frac{P(D=1 \mid Z=z) \cdot \frac{f_{Z}(z)}{P(D=1)}}{P(D=0 \mid Z=z) \cdot \frac{f_{Z}(z)}{P(D=0)}}=\frac{f_{Z \mid D=1}(z)}{f_{Z \mid D=0}(z)}
$$

\footnotetext{
${ }^{4}$ Naturally, we might as well adjust the comparison distribution and then compare the covariate-adjusted comparison distribution with the reference distribution. The two perspectives address the same question (i.e., how the relative distribution of $Y$ would look like if the two groups had the same distribution of $Z$ ) but give somewhat different answers. In the decomposition literature this is discussed as the "index problem" (see, e.g., Jann, 2008).

${ }^{5}$ To reweight the comparison group, we would use factor $1 / \Psi(z)$ instead of $\Psi(z)$.
} 
(using Bayes' theorem in the last step). The practical advantage of reweighting over integrating is that $\operatorname{Pr}(D=1 \mid Z=z)$ and, therefore, $\Psi(z)$ is relatively easy to estimate using binary choice models (e.g. logistic regression). ${ }^{6}$

In any case, whether we integrate over conditional distributions or whether we use reweighting, constructing counterfactual distributions in such a way assumes that the conditional distribution of $Y$ is "stable", that is, that the covariate distribution can be modified without changing the conditional distribution. However, even if such an exogeneity assumption is unrealistic in a given application, the "as if" scenarios based on counterfactual distributions can still be informative.

Furthermore, note that reweighting can be used as an alternative approach to identify location and shape effects (instead of applying adjustments as described in section 2.5), by modeling $\Psi$ as a function of $Y$. This is particularly useful if the analyzed outcome is categorical.

\section{Estimation}

For the following discussion, assume that there is a random sample of size $n$ for which we observe two variables, $X$ and $Y$. Furthermore, there is information on sampling weights $w$ as well as a (possibly empty) vector of covariates $Z$. That is, the data is $\left(Y_{i}, X_{i}, w_{i}, Z_{i}\right)$, $i=1, \ldots, n$. Set $w_{i}=1$ for all $i$ in case there are no sampling weights.

We intend to analyze the relative distribution of $X$ with respect to $Y$ between two subsamples. Let $D$ be an indicator for the comparison subsample $\left(D_{i}=1\right.$ if observation $i$ belongs to the comparison subsample, 0 else) and let $\mathcal{D}=\left\{i \mid D_{i}=1\right\}$ be the set of indices for which $D_{i}=1$. Likewise, let $R$ be an indicator for the reference subsample $\left(R_{i}=1\right.$ if observation $i$ belongs to the reference subsample, 0 else) and let $\mathcal{R}=\left\{i \mid R_{i}=1\right\}$ be the set of indices for which $R_{i}=1$. That is, we want to compare the distribution of $X$ in subsample $\mathcal{D}$ with the distribution of $Y$ in subsample $\mathcal{R}$.

We will use $F_{X \mid D}$ to denote the former, that is, the conditional distribution of $X$ given $D=1$, and $F_{Y \mid R}$ to denote the latter. In general, we will use letter " $D$ " for quantities related to $\mathcal{D}$ and letter " $R$ " for quantities related to $\mathcal{R}$. For example, $W_{D}=\sum D_{i} w_{i}$ and $W_{R}=\sum R_{i} w_{i}$ will be the sum of weights in the comparison sample and the reference sample, respectively. Furthermore, define $W=\sum w_{i}$ as the total sum of weights.

Note that $Y$ and $X$ may be the same and that $\mathcal{D}$ and $\mathcal{R}$ do not have to be distinct nor exhaustive. I use such a general setup to cover all possible cases. For example, if the subsamples are distinct and $Y=X$, then we are in a setting in which a single variable is compared between two groups (e.g., a comparison of wages from a sample of females to wages

\footnotetext{
${ }^{6}$ In their description of the implementation of relative distribution methods in R, Handcock and Aldrich (2002) conduct covariate-adjustment by resampling observations based on relative frequencies of covariate values. This is equivalent, in expectation, to reweighting the data by $\Psi(z)$.
} 
from a sample of males). Likewise, if $D=R$ and $Y \neq X$, we compare two variables within the same sample (e.g., a comparison of data on wages for the same individuals between two time points). Furthermore, if $X=Y$ and $\mathcal{D}$ is included in $\mathcal{R}$, then we compare the distribution of a variable in a subsample with the pooled distribution of that variable. Finally, if the union of $\mathcal{D}$ and $\mathcal{R}$ does not cover the whole sample (that is, if there are observations for which $D=R=0$ ), we are in a subpopulation estimation setting. Taking account of the observations that do not belong to the subpopulation may be important for standard error estimation.

\subsection{The relative distribution function}

To obtain an estimate for the relative $\mathrm{CDF}$

$$
G(r)=F_{X \mid D}\left(F_{Y \mid R}^{-1}(r)\right), \quad r \in[0,1]
$$

one can compute the relative rank of $X_{i}$ in distribution $F_{Y \mid R}$ for each $i \in \mathcal{D}$ and then take the value of the empirical CDF of these relative ranks at value $r$. That is, first compute

$$
\hat{r}_{i}=\frac{1}{W_{R}} \sum_{j \in \mathcal{R}} w_{j} \mathbb{1}\left\{Y_{j} \leq X_{i}\right\} \quad \text { for all } i \in \mathcal{D}
$$

where $\mathbb{1}\{a\}$ is the indicator function ( 1 if $a$ is true, 0 else). Then obtain the CDF as

$$
\widehat{G}(r)=\frac{1}{W_{D}} \sum_{i \in \mathcal{D}} w_{i} \mathbb{1}\left\{\hat{r}_{i} \leq r\right\}
$$

An issue with this simple computation is that it leads to a step function with jumps at distinct values of $\hat{r}$. Let $(i)$ refer to observations in $\mathcal{D}$ ordered by $\hat{r}$ such that $\hat{r}_{(1)} \leq \hat{r}_{(2)} \leq$ $\cdots \leq \hat{r}_{\left(n_{D}\right)}$. If $\hat{r}_{(i)}<r<\hat{r}_{(i+1)}$, that is, if evaluation point $r$ falls between two values of $\hat{r}$, then $\widehat{G}(r)$ will be equal to the CDF corresponding to the lower value of $\hat{r}$. Such behavior makes sense in case of an ordinary CDF. However, in the context of the relative distribution it appears more appropriate to linearly interpolate between the two points, as this is equivalent to breaking ties proportionally between the comparison distribution and the reference distribution. Hence, determine $\widehat{G}(r)$ as

$$
\widehat{G}(r)=\widehat{G}_{\left(i^{\prime}\right)}+\left(\widehat{G}_{\left(i^{\prime}+1\right)}-\widehat{G}_{\left(i^{\prime}\right)}\right) \frac{r-\hat{r}_{\left(i^{\prime}\right)}}{\hat{r}_{\left(i^{\prime}+1\right)}-\hat{r}_{\left(i^{\prime}\right)}}
$$

with

$$
\widehat{G}_{(i)}=\frac{1}{W_{D}} \sum_{j \in \mathcal{D}} w_{j} \mathbb{1}\left\{\hat{r}_{j} \leq \hat{r}_{(i)}\right\}
$$

where $i^{\prime}$ is selected such that $\hat{r}_{\left(i^{\prime}\right)}<r \leq \hat{r}_{\left(i^{\prime}+1\right)}$ (with $\hat{r}_{(0)}=\widehat{G}_{(0)}=0$ if $\hat{r}_{(1)}>0$ and $\hat{r}_{\left(n_{D}+1\right)}=\widehat{G}_{\left(n_{D}+1\right)}=1$ if $\left.\hat{r}_{\left(n_{D}\right)}<1\right)$. For values of $r$ that have an exact match in $\hat{r}_{i}, i \in \mathcal{D}$, 
this leads to the same result as (38). For $r$ values without exact match, (39) is equivalent to picking the result from a linear segmented curve connecting the points given by $\left(\widehat{G}_{(i)}, \hat{r}_{(i)}\right)$, $i=1, \ldots, n_{D}$.

Equation (39) improves on (38) in that it uses interpolation in regions where (38) is flat. It does not, however, take into account that flat regions in (38) may include outcome values that only exist in $F_{Y \mid R}$, nor does it take into account that there might be regions where the true $G(r)$ is upright due to outcome values that only occur in $F_{X \mid D}$. To handle these issues and obtain an estimate that exactly traces the observed data pattern, we can compute the empirical CDF for $F_{X \mid D}$ and $F_{Y \mid R}$ at each observed value in the data and then use linear interpolation to obtain $\widehat{G}(r)$. Let $\mathcal{Y}=\left\{y_{(1)}, \ldots, y_{(J)}\right\}$ be the ordered set of all distinct outcome values observed for $F_{X \mid D}$ and $F_{Y \mid R}$. We then compute

$$
\hat{r}_{(j)}^{D}=\frac{1}{W_{D}} \sum_{i \in \mathcal{D}} w_{i} \mathbb{1}\left\{X_{i} \leq y_{(j)}\right\} \quad \text { and } \quad \hat{r}_{(j)}^{R}=\frac{1}{W_{R}} \sum_{i \in \mathcal{R}} w_{i} \mathbb{1}\left\{Y_{i} \leq y_{(j)}\right\}
$$

for all $j=1, \ldots, J$, add origin $\hat{r}_{(0)}^{D}=\hat{r}_{(0)}^{R}=0$, and obtain the relative CDF as

$$
\widehat{G}(r)= \begin{cases}\hat{r}_{\left(j^{r}\right)}^{D} & \text { if } r=0 \\ \hat{r}_{\left(j_{r}\right)}^{D}\left(\hat{r}_{\left(j_{r}\right)}^{D}+\hat{r}_{\left(j^{r}\right)}^{D}\right) & \text { if } r=1 \\ 0.5 & \text { if } r=\hat{r}_{(j)}^{R} \text { for any } j \\ \hat{r}_{\left(j^{\prime}\right)}^{D}+\left(\hat{r}_{\left(j^{\prime}+1\right)}^{D}-\hat{r}_{\left(j^{\prime}\right)}^{D}\right) \frac{r-\hat{r}_{\left(j^{\prime}\right)}^{R}}{\hat{r}_{\left(j^{\prime}+1\right)}^{R}-\hat{r}_{\left(j^{\prime}\right)}^{R}} & \text { else }\end{cases}
$$

where $j_{r}$ and $j^{r}$ denote the smallest and largest value of $j$, respectively, for which $\hat{r}_{(j)}^{R}=r$, and where $j^{\prime}$ is chosen such that $\hat{r}_{\left(j^{\prime}\right)}^{R}<r<\hat{r}_{\left(j^{\prime}+1\right)}^{R}$. For graphical display we may also directly plot $\hat{r}_{(j)}^{D}$ against $\hat{r}_{(j)}^{R}$ and linearly connect the points. All estimates for $\widehat{G}(r)$ obtained using (41) will lie on that curve.

If all values in $\mathcal{Y}$ exist in both distributions, (41) will lead to the same results as (39). Furthermore, for continuous data, at least if the dataset is not very small, results from the two approaches will be very similar. Equation (41), however, leads to more appropriate results than (39) if the data is discrete.

\subsection{Computing relative ranks}

Relative density estimation and the estimation of summary measures of the relative distribution are typically implemented by analyzing the relative ranks of $X_{i}, i \in \mathcal{D}$, in distribution $F_{Y \mid R}$. A naïve approach is to compute the relative ranks using the values of the empirical CDF of $F_{Y \mid R}$, that is

$$
\hat{r}_{i}=\frac{1}{W_{R}} \sum_{j \in \mathcal{R}} w_{j} \mathbb{1}\left\{Y_{j} \leq X_{i}\right\}
$$


A problem with this approach is that the empirical CDF is a step function. This is particularly troublesome if there is heaping in the data such that there are large steps in the CDF, as is often the case with discrete data. One improvement is to use the so-called mid-distribution function instead of the regular CDF (Parzen, 2004) that deducts half a step size from the ranks in regions where the CDF is upright. Let

$$
\widehat{P}_{R}(Y=y)=\frac{1}{W_{R}} \sum_{j \in \mathcal{R}} w_{j} \mathbb{I}\left\{Y_{j}=y\right\}
$$

be the relative frequency of outcome $y$ in $F_{Y \mid R}$ (i.e. the step size in the CDF at value $y$ ). The relative ranks computed according to the mid-distribution function then are

$$
\hat{r}_{i}=\frac{1}{W_{R}} \sum_{j \in \mathcal{R}} w_{j} \mathbb{1}\left\{Y_{j} \leq X_{i}\right\}-\frac{1}{2} \widehat{P}_{R}\left(Y=X_{i}\right)
$$

Note that (44) differs from (42) only for observations that have ties in $F_{Y \mid R}$ (i.e. observations that hit a step). For all other observations $\widehat{P}_{R}$ is zero and hence the two computations lead to the same result. The relative mid-ranks are preferable over the naïve relative ranks because their average is exactly 0.5 if the two empirical distributions are identical. For the naïve relative ranks this does not hold; their average will be larger than 0.5 in this situation. That is, the naïve relative ranks have an upward bias. The size of the bias depends on how much heaping there is in the data. The more heaping, the larger the bias.

Using the mid-rank adjustment removes the bias in the relative ranks. Heaping, however, will still lead to undesirable results such as arbitrary spikes in the relative density estimate. A solution to this second issue is to break ties randomly and, hence, smooth out the step sizes of the CDF across tied observations. These broken relative ranks (including mid-rank adjustment) can be written as

$$
\hat{r}_{i}=\frac{1}{W_{R}} \sum_{j \in \mathcal{R}} w_{j} \mathbb{1}\left\{Y_{j} \leq X_{i}\right\}-\widehat{P}_{R}\left(Y=X_{i}\right) \frac{\widehat{P}_{D}\left(X=X_{i}\right)+0.5 w_{i}-\delta_{i}}{\widehat{P}_{D}\left(X=X_{i}\right)}
$$

where $\widehat{P}_{D}(X=y)$ is the relative frequency of outcome $y$ in $F_{X \mid D}$ and $\delta_{i}$ is the relative rank of $X_{i}$ among all ties of $X_{i}$ in $F_{X \mid D}$ when ties are broken randomly. Let $w_{1}^{(i)}, \ldots, w_{K_{i}}^{(i)}$ be the randomly ordered set of weights from the observations in $F_{X \mid D}$ that are equal to $X_{i}$ (including observation $i$ ), where $K_{i}$ is the size of the set (the order is kept stable across observations, that is, $w_{k}^{(i)}=w_{k}^{(j)}$ if $X_{i}=X_{j}$ ). Let $k_{i}$ be the position of observation $i$ in this set. The expression for $\delta_{i}$ then is

$$
\delta_{i}=\frac{1}{\sum_{k=1}^{K_{i}} w_{k}^{(i)}} \sum_{k=1}^{k_{i}} w_{k}^{(i)}
$$

which simplifies to $\delta_{i}=k_{i} / K_{i}$ if the weights are constant. ${ }^{7}$

\footnotetext{
${ }^{7}$ Due to the random ordering, repeated computation of (45) will lead to slightly different results for the relative density and other estimates unless the weights are constant (or unless there are no ties). One
} 
To obtain broken relative ranks without mid-rank adjustment, set $0.5 w_{i}$ in (45) to zero. Whereas the mid-rank adjustment can have a strong effect on results if relative ranks are computed without breaking ties (equation 42 vs. equation 44), the adjustment is only of minor importance in (45), because breaking ties makes the individual step sizes small (unless there is large variation in weights).

For location-adjusted relative ranks, the same equations can be applied to appropriately transformed input variables. For example, to compute the relative ranks based on a locationadjusted reference distribution, use

$$
\tilde{Y}=Y-\hat{\mu}_{Y \mid R}+\hat{\mu}_{X \mid D}
$$

instead of $Y$ in the above equations, where $\hat{\mu}_{Y \mid R}$ is the median or mean of $Y$ in subsample $\mathcal{R}$ and $\hat{\mu}_{X \mid D}$ is the median or mean of $X$ in subsample $\mathcal{D}$. Location-and-scale, multiplicative, or logarithmic adjustments can be handled analogously.

In contrast, for shape adjustment, one of the distributions has to be swapped. For example, to compute the relative ranks based on a shape-adjusted comparison distribution (i.e., a comparison distribution that has the same shape as the reference distribution, but a different location), use

$$
\widetilde{X}=Y-\hat{\mu}_{Y \mid R}+\hat{\mu}_{X \mid D}
$$

instead of $X$ and then set the comparison sample to $\widetilde{\mathcal{D}}=\mathcal{R}$ instead of $\mathcal{D}$.

\subsection{The relative density function}

\subsubsection{Kernel density estimation for continuous data}

Estimation of the relative density can be implemented by applying a univariate density estimator to the relative ranks (preferably as defined in equation 45). Compared to a standard density estimation problem, there are two specific complications that should to be taken into account. First, the support of the relative density is bounded at 0 and 1 . Standard density estimators, however, are designed such that they smoothly approach zero outside the support of the observed data, which leads to an underestimation of the density at the boundaries. Second, automatic bandwidth selection should be adapted to take account of the specific nature of relative data.

Given an evaluation point $r \in[0,1]$, a kernel density estimate of the relative density can be written as

$$
\hat{g}(r)=\frac{1}{W_{D}} \sum_{i \in \mathcal{D}} w_{i} K_{c}\left(r, \hat{r}_{i}, h\right)
$$

(arbitrary) solution to enforce stable results is to sort the observations within ties in (ascending or descending) order of the weights. 
where $K_{c}\left(r, \hat{r}_{i}, h\right)$ is a boundary-corrected kernel function with bandwidth $h$. For example, the renormalization technique uses

$$
K_{c}\left(r, \hat{r}_{i}, h\right)=\frac{1}{h} K\left(\frac{r-\hat{r}_{i}}{h}\right) c(r, h) \quad \text { with } \quad c(r, h)=\left(\int_{(0-r) / h}^{(1-r) / h} K(x) \mathrm{d} x\right)^{-1}
$$

where $K(x)$ is a standard kernel function such as the Gaussian kernel. The logic of the procedure is to rescale the density estimate by the inverse of the area of the kernel function that lies within the support of $r$. For some alternative boundary-correction techniques see Jann (2007).

The bandwidth $h$ that determines the degree of smoothing (larger values for $h$ lead to a smoother density function) can either be set manually or be determined automatically from the data. Various suggestions for automatic bandwidth selectors exist in the literature, some based on crude rules-of-thumb, some employing more sophisticated procedures (see Jann, 2007 for an overview of some of the suggestions). For relative density estimation, these standard bandwidth selectors should be adapted to take account of the specific nature of relative data. Suggestions for appropriate modifications are given by Ćwik and Mielniczuk (1993). The reldist command below supports several automatic bandwidth selectors, but we refrain from discussing their details here. ${ }^{8}$

\subsubsection{Histogram density estimation}

A complement to kernel density estimation is to obtain a histogram of the relative density. Let $(a, b]$ be an interval on the support of $r$. The histogram density estimate for that interval can then be obtained as

$$
\hat{g}(a, b)=\frac{\widehat{P}_{D}(a<r \leq b)}{b-a}=\frac{1}{W_{D}} \sum_{i \in \mathcal{D}} w_{i} \frac{\mathbb{1}\left\{a<\hat{r}_{i} \leq b\right\}}{b-a}
$$

(with a modification in case of $a=0$ such that the interval includes the lower bound). A convenient setup is to split the support of $r$ into $K$ evenly sized bins defining the intervals $\left[0, \frac{1}{K}\right],\left(\frac{1}{K}, \frac{2}{K}\right], \ldots,\left(\frac{k-1}{K}, \frac{k}{K}\right], \ldots,\left(\frac{K-1}{K}, 1\right]$, such that each bin covers $\frac{1}{K}$ th of the reference distribution.

\footnotetext{
${ }^{8}$ Estimator 47 uses a global bandwidth that is constant across observations. A popular alternative is the adaptive estimator based on a varying bandwidth depending on the local density of the data (Abramson, 1982). For the adaptive estimator, replace $h$ by $h_{i}=h \cdot \sqrt{\widetilde{g}^{0} / \hat{g}^{0}\left(\hat{r}_{i}\right)}$ where $\hat{g}^{0}\left(\hat{r}_{i}\right)$ is an initial (constantbandwidth) density estimate and $\widetilde{g}^{0}$ is the geometric mean of $\hat{g}^{0}\left(\hat{r}_{i}\right)$ over all observations in $\mathcal{D}$. The procedure may be iterated several times (each time using the density estimate from the last step to determine the new $h_{i}$ ), but typically additional iterations do not change the estimate much. The adaptive estimator is attractive for regular density estimation because there is a one-to-one relation between the density and the local sample size. For the relative density, however, the local sample size is constant for one of the groups (the reference group), such that the adaptive estimator appears less convincing.
} 
The histogram density has an intuitive interpretation. For example, a value of 2 means that the fraction of the comparison distribution that falls into the bin is twice as large as the fraction of the reference distribution. In other words, the comparison distribution is overrepresented in the bin by a factor of 2 . A value of 0.5 means that the proportion of the comparison distribution is only half the proportion of the reference distribution. A kernel density estimate of the relative ranks has, in principle, the same meaning (it shows the relative over- or underrepresentation multiplier at each level of $r$ ), but the explicit binning may make the histogram more easy to interpret.

\subsubsection{Discrete relative density for categorical data}

For categorical data, the relative density can be computed directly from the relative probabilities across the levels of the data. Without loss of generality, let $k=1, \ldots, K$ be these levels. The relative density for level $k$ is then estimated as

$$
\hat{g}_{k}=\frac{\widehat{P}_{D}(X=k)}{\widehat{P}_{R}(Y=k)}
$$

with

$$
\widehat{P}_{D}(X=k)=\frac{1}{W_{D}} \sum_{i \in \mathcal{D}} w_{i} \mathbb{1}\left\{X_{i}=k\right\} \quad \text { and } \quad \widehat{P}_{R}(Y=k)=\frac{1}{W_{R}} \sum_{i \in \mathcal{R}} w_{i} \mathbb{1}\left\{Y_{i}=k\right\}
$$

Discrete relative density $\hat{g}_{k}$ is well defined only for levels $k$ that exist in the reference distribution.

When plotting the relative density for categorical data, $\hat{g}_{k}$ can be plotted against $\widehat{P}_{R}(Y \leq$ $k$ ) using a step function, including an additional point at coordinate $\left(\hat{g}_{1}, 0\right)$ for the first step. Alternatively, the density can be plotted using a histogram with bar widths equal to $\widehat{P}_{R}(Y=k)$ and bar midpoints equal to $\widehat{P}_{R}(Y \leq k)-\widehat{P}_{R}(Y=k) / 2$.

\subsection{Divergence}

\subsubsection{Continuous data}

To estimate the $\chi^{2}$, Kullback-Leibler, and dissimilarity measures, obtain an estimate of the relative density over a grid of evaluation points and then "integrate" the result. For example, let $r_{k}=k / K-1 /(2 K), k=1, \ldots, K$, be a regular grid of evaluation points spanning the support of $r$ from $1 /(2 K)$ to $1-1 /(2 K)$. The divergence measures can then be estimated as

$$
\widehat{\chi}^{2}=\frac{1}{K} \sum_{k=1}^{K}\left(\hat{g}\left(r_{k}\right)-1\right)^{2} \quad \widehat{\mathrm{KL}}=\frac{1}{K} \sum_{k=1}^{K} \hat{g}\left(r_{k}\right) \ln \left(\hat{g}\left(r_{k}\right)\right) \quad \widehat{\mathrm{TVD}}=\frac{1}{2 K} \sum_{k=1}^{K}\left|\hat{g}\left(r_{k}\right)-1\right|
$$


where $\hat{g}\left(r_{k}\right)$ is the density estimate at evaluation point $r_{k}$ (that is, the integral is approximated by using a rectangle of width $1 / K$ around each evaluation point). The size of the evaluation grid should not matter too much for the results, as long as it is sufficiently dense. However, results may strongly depend on the bandwidth used for density estimation. Divergence measures will typically increase with a decrease in the bandwidth. Stated differently, more smoothing leads to lower divergence. In general, TVD is less sensitive in this regard than the other two measures.

An alternative is to obtain the divergence measures from a histogram of the relative density. Assuming $K$ evenly sized bins covering the whole range of $r$, the histogram-based estimates of the divergence measures can be obtained using (51) with $\hat{g}\left(r_{k}\right)$ replaced by the histogram estimate of the relative density in bin $k$. Results may strongly depend on the number of bins.

\subsubsection{Categorical data}

Divergence measures for categorical data can be defined in terms of the categorical relative density as introduced above. Let $k=1, \ldots, K$ be the levels of the data. The divergence estimates then are

$$
\widehat{\chi}^{2}=\sum_{k=1}^{K} \frac{\left(\hat{p}_{k}^{D}-\hat{p}_{k}^{R}\right)^{2}}{\hat{p}_{k}^{R}} \quad \widehat{\mathrm{KL}}=\sum_{k=1}^{K} \hat{p}_{k}^{D} \ln \left(\frac{\hat{p}_{k}^{D}}{\hat{p}_{k}^{R}}\right) \quad \widehat{\mathrm{TVD}}=\sum_{k=1}^{K} \frac{1}{2}\left|\hat{p}_{k}^{D}-\hat{p}_{k}^{R}\right|
$$

where $\hat{p}_{k}^{D}=\widehat{P}_{D}(X=k)$ and $\hat{p}_{k}^{R}=\widehat{P}_{R}(Y=k)$

\subsection{Median relative polarization}

For the polarization indices first compute location-adjusted relative ranks using one of the above methods, where the median is used as location measure. Let $\hat{\tilde{r}}_{i}$ be these locationadjusted ranks. Whether we transform the reference data or the comparison data does not matter. An estimate for MRP can then be obtained as

$$
\widehat{\mathrm{MRP}}=\left(\frac{4}{W_{D}} \sum_{i \in \mathcal{D}} w_{i}\left|\hat{\tilde{r}}_{i}-0.5\right|\right)-1
$$

Furthermore, using

$$
\begin{aligned}
& \widehat{\mathrm{LRP}}=\left(\frac{8}{W_{D}} \sum_{i \in \mathcal{D}} w_{i}\left|\hat{\tilde{r}}_{i}-0.5\right| \mathbb{1}\left\{\hat{\tilde{r}}_{i}<.5\right\}\right)-1 \\
& \widehat{\mathrm{URP}}=\left(\frac{8}{W_{D}} \sum_{i \in \mathcal{D}} w_{i}\left|\hat{\tilde{r}}_{i}-0.5\right| \mathbb{1}\left\{\hat{\tilde{r}}_{i}>.5\right\}\right)-1
\end{aligned}
$$


as estimates for LRP and URP ensures that

$$
\widehat{\mathrm{MRP}}=\frac{\widehat{\mathrm{LRP}}+\widehat{\mathrm{URP}}}{2}
$$

Note that, in theory, the MRP of $F_{X \mid D}$ with respect to $F_{Y \mid R}$ is equal to - MRP of $F_{Y \mid R}$ with respect to $F_{X \mid D}$. In practice, however, heaping in the data may cause the median of the location-adjusted relative ranks to differ from 0.5 and, hence, cause this relation to be violated. Applying mid-distribution correction and breaking ties when computing the ranks typically reduces the discrepancy, but may not entirely remove it.

\subsection{Covariate balancing}

Assume that $\mathcal{D}$ and $\mathcal{R}$ are distinct and exhaustive, such that $D$ is an indicator for the comparison group $(D=1)$ versus the reference group $(D=0)$. A simple approach for covariate-adjustment by reweighting is to run a logistic regression of $D$ on $Z$ and obtain predictions $\hat{p}_{i}=\widehat{P}\left(D=1 \mid Z=Z_{i}\right)$ from the model. To reweight the reference group, define adjusted weights

$$
\widetilde{w}_{i}= \begin{cases}w_{i} \frac{\hat{p}_{i}}{1-\hat{p}_{i}} c_{R} & \text { if } i \in \mathcal{R} \\ w_{i} & \text { else }\end{cases}
$$

where $c_{R}=W_{R} / \sum_{i \in \mathcal{R}} w_{i} \frac{\hat{p}_{i}}{1-\hat{p}_{i}}$ is a scaling factor ensuring that the group size (that is, its sum of weights) remains constant, and use these weights in all computations instead of the original weights. Likewise, to reweight the comparison group, define the adjusted weights as

$$
\widetilde{w}_{i}= \begin{cases}w_{i} \frac{1-\hat{p}_{i}}{\hat{p}_{i}} c_{D} & \text { if } i \in \mathcal{D} \\ w_{i} & \text { else }\end{cases}
$$

with $c_{D}=W_{D} / \sum_{i \in \mathcal{D}} w_{i} \frac{1-\hat{p}_{i}}{\hat{p}_{i}}$. The described procedure is equivalent to what is known as "inverse probability weighing" (IPW) in the causal inference literature (see [TE] teffects ipw). Any other approach to obtain balancing weights may do as well. See, for example, kmatch (Jann, 2017) for techniques such as entropy balancing or matching.

\subsection{Standard errors}

The following exposition on standard errors is rather technical and can safely be ignored by readers who are only interested in the practical application of the methods; it is not needed for obtaining an understanding of relative distribution methods and for being able to correctly apply the software and interpret the results. Nonetheless, I consider the presented material an important and original contribution that cannot be found elsewhere in the literature. I first illustrate how influence functions can be obtained by analogy to the method of moments and then derive specific expressions for all relative distribution quantities of interest, including possible covariate adjustment. 


\subsubsection{Variance estimation by means of influence functions}

Influence functions (Hampel, 1974) provide a convenient approach to estimate the sampling variances of the different statistics discussed above. Intuitively, an influence function is an approximation of how a functional of a distribution changes once some data mass is added at a specific point in the distribution. Random sampling can be seen as a process that modifies the distribution in such a way and, hence, leads to variation in statistics computed from the distribution. It can be shown that, asymptotically, this variation (i.e., the sampling variance) is equal to the expectation of the square of the influence function divided by the sample size (e.g. Deville, 1999). Therefore, to obtain an estimate of the sampling variance from a given sample, we can evaluate the influence function at each observation in the data and then compute the sampling variance of the mean of these values using textbook formulas. ${ }^{9}$ More generally, once influence functions are available for a set of statistics, the variance matrix of these statistics can be obtained by taking a mean estimate (using $[\mathrm{R}]$ mean) of the influence functions (or a total estimate using $[\mathrm{R}]$ total, depending on the scaling of the influence functions). Sampling weights or other complex survey characteristics do not change the form of the influence function and can be taken into account when computing the mean (or total) estimate. This makes the influence function approach very general.

One-parameter setting There is a close connection between influence functions and the method of moments (see Jann, 2020). Let $h_{i}^{\theta}$ be the moment condition for estimating $\theta$ in a simple one-parameter setting, such that $\hat{\theta}$ satisfies

$$
0=\frac{1}{W} \sum_{i=1}^{n} w_{i} \hat{h}_{i}^{\theta}
$$

where $\hat{h}_{i}^{\theta}$ denotes $h_{i}^{\theta}$ with $\theta$ set to $\hat{\theta}$. Observation $i$ 's value of the empirical influence function of $\hat{\theta}$ can then be obtained as

$$
\operatorname{IF}_{i}(\hat{\theta})=\frac{1}{-\widehat{\Delta}^{\theta}} \hat{h}_{i}^{\theta}
$$

where

$$
\widehat{\Delta}^{\theta}=\left.\frac{1}{W} \sum_{i=1}^{n} w_{i} \frac{\partial h_{i}^{\theta}}{\partial \theta}\right|_{\theta=\hat{\theta}}
$$

is an estimate of the expectation of the derivative of $h^{\theta}$ at point $\theta=\hat{\theta}$. Consider the mean estimator

$$
\hat{\bar{y}}=\frac{1}{W} \sum_{i=1}^{n} w_{i} Y_{i}
$$

for which the moment condition is given as

$$
h_{i}^{\bar{y}}=Y_{i}-\bar{y}
$$

\footnotetext{
${ }^{9}$ Since the mean of an influence function is zero by definition, the expectation of the squared influence function is equal to the variance of the influence function.
} 
Since

$$
\widehat{\Delta}^{\bar{y}}=\left.\frac{1}{W} \sum_{i=1}^{n} w_{i} \frac{\partial h_{i}^{\bar{y}}}{\partial \bar{y}}\right|_{\bar{y}=\hat{y}}=\frac{1}{W} \sum_{i=1}^{n} w_{i}(-1)=-1
$$

the influence function simplifies to

$$
\mathrm{IF}_{i}(\hat{\bar{y}})=Y_{i}-\hat{\bar{y}}
$$

Assuming a survey design without clustering or stratification, the sampling variance of $\hat{\bar{y}}$ can then be estimated as

$$
\widehat{V}(\hat{\bar{y}})=\frac{1}{W(W-W / n)} \sum_{i=1}^{n} w_{i}^{2}\left(\operatorname{IF}_{i}(\hat{\bar{y}})\right)^{2}
$$

This is equivalent to the textbook formula for the variance of the mean, as can easily be seen if $\operatorname{IF}_{i}(\hat{\bar{y}})$ is replaced by its definition. The general point is that we can use the same variance formula also in other situations. That is, the variance of a statistic can be obtained by applying the above formula (or a variant if it depending on survey design) to its influence function, whatever the statistic might be.

Multiple-parameter setting Deriving the influence function becomes more involved if a statistic includes auxiliary parameters that are estimated from the data. Think of a system of equations with moment conditions $h^{\theta_{1}}, h^{\theta_{2}}, \ldots, h^{\theta_{p}}$ where $\theta_{1}$ depends on $\theta_{2}, \ldots, \theta_{p}$ (that is, all $\theta_{j}$ appear as arguments in the moment condition for $\theta_{1}$ ). The influence function for $\theta_{1}$ can then be written as

$$
\operatorname{IF}_{i}\left(\hat{\theta}_{1}\right)=\frac{1}{-\widehat{\Delta}^{\theta_{1}}}\left(\hat{h}_{i}^{\theta_{1}}+\sum_{j=2}^{p} \widehat{\Delta}_{\theta_{j}}^{\theta_{1}} \mathrm{IF}_{i}\left(\hat{\theta}_{j}\right)\right)
$$

where $\hat{h}_{i}^{\theta_{1}}$ denotes the value of $h_{i}^{\theta_{1}}$ with $\theta=\left(\theta_{1}, \ldots, \theta_{p}\right)$ set to $\hat{\theta}=\left(\hat{\theta}_{1}, \ldots, \hat{\theta}_{p}\right)$ and

$$
\widehat{\Delta}_{\theta_{j}}^{\theta_{1}}=\left.\frac{1}{W} \sum_{i=1}^{n} w_{i} \frac{\partial h_{i}^{\theta_{1}}}{\partial \theta_{j}}\right|_{\theta=\hat{\theta}}
$$

is an estimate of the expectation of the partial derivative of $h^{\theta_{1}}$ by $\theta_{j}$ at point $\hat{\theta}$. If parameters $\theta_{j}, j \geq 2$, themselves depend on further parameters, their influence functions will have an analogous form. That is, multiple-parameter problems can be solved recursively by applying equation (63) repeatedly.

One implication of (63) is that, if $\gamma=t(\theta)$, where $t(\theta)$ is a simple transformation function of $\theta=\left(\theta_{1}, \ldots, \theta_{p}\right)$ that does not involve the data (i.e. a linear or nonlinear combination of the elements in $\theta$ ), the influence function for $\hat{\gamma}$ can be written as

$$
\operatorname{IF}_{i}(\hat{\gamma})=\left.\frac{\partial t(\theta)}{\partial \theta_{1}}\right|_{\theta=\hat{\theta}} \operatorname{IF}_{i}\left(\hat{\theta}_{1}\right)+\cdots+\left.\frac{\partial t(\theta)}{\partial \theta_{p}}\right|_{\theta=\hat{\theta}} \operatorname{IF}_{i}\left(\hat{\theta}_{p}\right)
$$

This means that the influence function of a statistic that is defined as an aggregate of other statistics can be obtained as an aggregate of the influence functions of these statistics. 
Subpopulation estimation Because the relative size of a subsample is subject to sampling error, influence functions should always be evaluated for all observations in the data, also when only a subpopulation is analyzed (even though for many statistics this may not change the results). Furthermore, the relative distribution is typically computed using data from two subsamples, so that the influence functions bellow will inherently contain multiple components based on different observations. Using a full-sample approach is thus inevitable. Subpopulation influence functions defined in terms of all observations can be obtained by including appropriate subpopulation indicators in the moment conditions.

Consider the influence function for subpopulation mean

$$
\hat{\bar{y}}_{S}=\frac{1}{W_{S}} \sum_{i \in \mathcal{S}} w_{i} S_{i} Y_{i}
$$

where $S_{i}$ is an indicator for whether observation $i$ belongs to subsample $\mathcal{S}$ and $W_{S}$ is the sum of weights in the subsample. The full-sample moment condition for $\hat{\bar{y}}_{S}$ can be written as

$$
h_{i}^{\bar{y}_{S}}=S_{i}\left(Y_{i}-\bar{y}_{S}\right)
$$

Since

$$
\widehat{\Delta}^{\bar{y} S}=\frac{1}{W} \sum_{i=1}^{n} w_{i}\left(-S_{i}\right)=-\frac{W_{S}}{W}
$$

the influence function for $\hat{\bar{y}}_{S}$ becomes

$$
\operatorname{IF}_{i}\left(\hat{\bar{y}}_{S}\right)=\frac{W}{W_{S}} S_{i}\left(Y_{i}-\hat{\bar{y}}_{S}\right)
$$

The influence function will be zero for observations outside subsample $\mathcal{S}$. However, taking the standard error of the mean of this influence function across all observations will provide a consistent standard error for $\hat{\bar{y}}_{S}$.

In practice it may be convenient to omit the global $W$ from the definition of the influence function and only divide by the relevant subpopulation size. In this case, the appropriate standard error is provided by the standard error of the total of the influence function. An advantage of defining influence functions in this way is that they can be computed from the subsample data alone, without knowing the total sum of weights.

Overview of influence functions for various statistics Using the methods above, we can obtain influence functions for various statistics that are relevant in the context of relative distribution analysis. Table 1 provides an overview. The influence functions have been derived for statistics that are conditional on $S=1$, where $S$ is an indicator for whether an observation belongs to subsample $\mathcal{S}$. For unconditional statistics, set $S$ to 1 for all observations. 
The variance is an example of a multi-parameter statistic, but it is a special case because the influence function for the auxiliary parameter drops out of the equation. Furthermore, note that a quantile can be defined as the value $\hat{q}_{Y}(p)$ that solves

$$
0=\frac{1}{W} \sum_{i=1}^{n} w_{i}\left(\mathbb{1}\left\{Y_{i} \leq q_{Y}(p)\right\}-p\right)
$$

so that $\hat{f}_{Y}\left(\hat{q}_{Y}(p)\right)$ provides an estimate of the expectation of the derivative of the relevant moment condition.

\subsubsection{Influence function for the relative CDF}

Using notation as introduced at the start of this section, the empirical CDF of the relative ranks can be written as

$$
\widehat{G}(r)=\widehat{F}_{X \mid D}\left(\hat{q}_{r}\right)=\frac{1}{W_{D}} \sum_{i \in \mathcal{D}} w_{i} \mathbb{1}\left\{X_{i} \leq \hat{q}_{r}\right\}
$$

where $\hat{q}_{r}$ is shorthand notation for $\hat{q}_{Y \mid R}(r)=\widehat{F}_{Y \mid R}^{-1}(r)$. The moment conditions for $G(r)$ and $q_{r}$ are

$$
\begin{aligned}
h_{i}^{G} & =D_{i}\left(\mathbb{1}\left\{X_{i} \leq q_{r}\right\}-G(r)\right) \\
h_{i}^{q} & =R_{i}\left(\mathbb{1}\left\{Y_{i} \leq q_{r}\right\}-r\right)
\end{aligned}
$$

Working through (63) yields

$$
\operatorname{IF}_{i}(\widehat{G}(r))=\frac{W}{W_{D}} \hat{h}_{i}^{G}+\hat{f}_{X \mid D}\left(\hat{q}_{r}\right) \operatorname{IF}_{i}\left(\hat{q}_{r}\right)
$$

Since, according to table 1,

$$
\operatorname{IF}_{i}\left(\hat{q}_{r}\right)=\frac{W}{W_{R}} R_{i}\left(\frac{r-\mathbb{1}\left\{Y_{i} \leq \hat{q}_{r}\right\}}{\hat{f}_{Y \mid R}\left(\hat{q}_{r}\right)}\right)
$$

equation (71) can be written as

$$
\operatorname{IF}_{i}(\widehat{G}(r))=W \frac{D_{i}}{W_{D}}\left(\mathbb{1}\left\{X_{i} \leq \hat{q}_{r}\right\}-\widehat{G}(r)\right)+W \frac{R_{i}}{W_{R}} \frac{\hat{f}_{X \mid D}\left(\hat{q}_{r}\right)}{\hat{f}_{Y \mid R}\left(\hat{q}_{r}\right)}\left(r-\mathbb{1}\left\{Y_{i} \leq \hat{q}_{r}\right\}\right)
$$

The density ratio in the second term is equal to the relative density by definition, so we could replace it by $\hat{g}(r)$. Both variants should yield a consistent standard error estimate. 
Table 1: Influence functions for various statistics

\begin{tabular}{ll}
\hline Statistic $($ conditional on $S=1)$ & Empirical influence function \\
\hline Mean & $\mathrm{IF}_{i}=\frac{W}{W_{S}} S_{i}\left(Y_{i}-\hat{\bar{y}}_{S}\right)$ \\
$\hat{\bar{y}}_{S}=\frac{1}{W_{S}} \sum_{i \in \mathcal{S}} w_{i} Y_{i}$ & \\
Empirical CDF & $\mathrm{IF}_{i}=\frac{W}{W_{S}} S_{i}\left(\mathbb{1}\left\{Y_{i} \leq y\right\}-\widehat{F}_{Y \mid S}(y)\right)$ \\
$\widehat{F}_{Y \mid S}(y)=\frac{1}{W_{S}} \sum_{i \in \mathcal{S}} w_{i} \mathbb{1}\left\{Y_{i} \leq y\right\}$ &
\end{tabular}

Kernel PDF (assuming $h$ fixed)

$$
\hat{f}_{Y \mid S}(y)=\frac{1}{W_{S}} \sum_{i \in \mathcal{S}} w_{i} \frac{1}{h} K\left(\frac{y-Y_{i}}{h}\right) \quad \mathrm{IF}_{i}=\frac{W}{W_{S}} S_{i}\left(\frac{1}{h} K\left(\frac{y-Y_{i}}{h}\right)-\hat{f}_{Y \mid S}(y)\right)
$$

Histogram PDF

$$
\hat{f}_{Y \mid S}(a, b)=\frac{1}{W_{S}} \sum_{i \in \mathcal{S}} w_{i} \frac{\mathbb{1}\left\{a<Y_{i} \leq b\right\}}{b-a} \quad \mathrm{IF}_{i}=\frac{W}{W_{S}} S_{i}\left(\frac{\mathbb{1}\left\{a<Y_{i} \leq b\right\}}{b-a}-\hat{f}_{Y \mid S}(a, b)\right)
$$

Quantile

$$
\hat{q}_{Y \mid S}(p)=\widehat{F}_{Y \mid S}^{-1}(p) \quad \quad \mathrm{IF}_{i}=\frac{W}{W_{S}} S_{i}\left(\frac{p-\mathbb{1}\left\{Y_{i} \leq \hat{q}_{Y \mid S}(p)\right\}}{\hat{f}_{Y \mid S}\left(\hat{q}_{Y \mid S}(p)\right)}\right)
$$

Median

$$
\hat{\tilde{y}}_{S}=\hat{q}_{Y \mid S}(0.5)
$$

$$
\mathrm{IF}_{i}=\frac{W}{W_{S}} S_{i}\left(\frac{.5-\mathbb{1}\left\{Y_{i} \leq \hat{\tilde{y}}_{S}\right\}}{\hat{f}_{Y \mid S}\left(\hat{\tilde{y}}_{S}\right)}\right)
$$

Variance

$$
\hat{\sigma}_{Y \mid S}^{2}=\frac{1}{W_{S}-\frac{W_{S}}{n_{S}}} \sum_{i \in \mathcal{S}} w_{i}\left(Y_{i}-\hat{\bar{y}}_{S}\right)^{2} \quad \mathrm{IF}_{i}=\frac{W}{W_{S}} S_{i}\left(c \cdot\left(Y_{i}-\hat{\bar{y}}_{S}\right)^{2}-\hat{\sigma}_{Y \mid S}^{2}\right), c=\frac{1}{1-\frac{1}{n_{S}}}
$$

Standard deviation

$$
\hat{\sigma}_{Y \mid S}=\sqrt{\hat{\sigma}_{Y \mid S}^{2}}
$$

$$
\mathrm{IF}_{i}=\frac{W}{W_{S}} S_{i}\left(\frac{c \cdot\left(Y_{i}-\hat{\bar{y}}_{S}\right)^{2}-\hat{\sigma}_{Y \mid S}^{2}}{2 \hat{\sigma}_{Y \mid S}}\right), c=\frac{1}{1-\frac{1}{n_{S}}}
$$

Interquartile range

$$
\operatorname{IQR}_{Y \mid S}=\hat{q}_{Y \mid S}(0.75)-\hat{q}_{Y \mid S}(0.25) \quad \operatorname{IF}_{i}=\operatorname{IF}_{i}\left(\hat{q}_{Y \mid S}(0.75)\right)-\operatorname{IF}_{i}\left(\hat{q}_{Y \mid S}(0.25)\right)
$$


Location and scale adjustment For the relative CDF based on location (and, possibly, scale) adjusted data, replace $q_{r}$ by $\tilde{q}_{r}=t\left(q_{r}, \theta\right)$ in the above formulas, where $t(y, \theta)$ is a scalar transformation function depending on a set of location and scale parameters $\theta=\left(\theta_{1}, \ldots, \theta_{K}\right)$. More specifically, if $t_{D}(y, \theta)$ is the transformation function applied to the comparison data and $t_{R}(y, \theta)$ is the transformation function applied to the reference data, we have

$$
\tilde{q}_{r}=t\left(q_{r}, \theta\right)=t_{D}^{-1}\left(t_{R}\left(q_{r}, \theta\right), \theta\right)
$$

The adjusted relative CDF then becomes

$$
\widehat{\widetilde{G}}(r)=\widehat{F}_{D}\left(\hat{\tilde{q}}_{r}\right)=\frac{1}{W_{D}} \sum_{i \in \mathcal{D}} w_{i} \mathbb{1}\left\{X_{i} \leq \hat{\tilde{q}}_{r}\right\}
$$

with $\hat{\tilde{q}}_{r}=t\left(\hat{q}_{r}, \hat{\theta}\right)$ and $\hat{q}_{r}=\widehat{F}_{Y \mid R}^{-1}(r)$, such that the influence function can then be written as

$$
\operatorname{IF}_{i}(\widehat{\widetilde{G}}(r))=\frac{W}{W_{D}} \hat{h}_{i}^{\widetilde{G}}+\hat{f}_{X \mid D}\left(\hat{\tilde{q}}_{r}\right) \operatorname{IF}_{i}\left(\hat{\tilde{q}}_{r}\right)
$$

with

$$
h_{i}^{\widetilde{G}}=D_{i}\left(\mathbb{1}\left\{X_{i} \leq \tilde{q}_{r}\right\}-\widetilde{G}(r)\right)
$$

and

$$
\mathrm{IF}_{i}\left(\hat{\tilde{q}}_{r}\right)=\tau_{q_{r}} \mathrm{IF}_{i}\left(\hat{q}_{r}\right)+\sum_{k=1}^{K} \tau_{\theta_{k}} \mathrm{IF}_{i}\left(\hat{\theta}_{k}\right)
$$

where

$$
\tau_{q_{r}}=\left.\frac{\partial t\left(q_{r}, \theta\right)}{\partial q_{r}}\right|_{q_{r}=\hat{q}_{r}, \theta=\hat{\theta}} \quad \text { and } \quad \tau_{\theta_{k}}=\left.\frac{\partial t\left(q_{r}, \theta\right)}{\partial \theta_{k}}\right|_{q_{r}=\hat{q}_{r}, \theta=\hat{\theta}}
$$

For example, in case of an additive location adjustment (of either the reference distribution or the comparison distribution) we have $t\left(q_{r}, \theta\right)=q_{r}-\mu_{Y \mid R}+\mu_{X \mid D}$ such that

$$
\operatorname{IF}_{i}\left(\hat{\tilde{q}}_{r}\right)=\operatorname{IF}_{i}\left(\hat{q}_{r}\right)-\operatorname{IF}_{i}\left(\hat{\mu}_{Y \mid R}\right)+\operatorname{IF}_{i}\left(\hat{\mu}_{X \mid D}\right)
$$

where expressions for the three influence functions included in $\operatorname{IF}_{i}\left(\hat{\tilde{q}}_{r}\right)$ can be found in table 1 ( $\mu$ is either the median or the mean). Likewise, in case of a multiplicative adjustment, we have $t\left(q_{r}, \theta\right)=q_{r} \cdot \mu_{X \mid D} / \mu_{Y \mid R}$, such that

$$
\operatorname{IF}_{i}\left(\hat{\tilde{q}}_{r}\right)=\frac{\hat{\mu}_{X \mid D}}{\hat{\mu}_{Y \mid R}} \operatorname{IF}_{i}\left(\hat{q}_{r}\right)+\frac{\hat{q}_{r}}{\hat{\mu}_{Y \mid R}} \operatorname{IF}_{i}\left(\hat{\mu}_{X \mid D}\right)-\frac{\hat{q}_{r} \hat{\mu}_{X \mid D}}{\left(\hat{\mu}_{Y \mid R}\right)^{2}} \operatorname{IF}_{i}\left(\hat{\mu}_{Y \mid R}\right)
$$

In case of additive location and scale adjustment, we have $t\left(q_{r}, \theta\right)=\left(q_{r}-\mu_{Y \mid R}\right) \cdot s_{X \mid D} / s_{Y \mid R}+$ $\mu_{X \mid D}$, such that

$$
\begin{aligned}
\operatorname{IF}_{i}\left(\hat{\tilde{q}}_{r}\right)= & \frac{\hat{s}_{X \mid D}}{\hat{s}_{Y \mid R}} \operatorname{IF}_{i}\left(\hat{q}_{r}\right)-\frac{\hat{s}_{X \mid D}}{\hat{s}_{Y \mid R}} \operatorname{IF}_{i}\left(\hat{\mu}_{Y \mid R}\right)+\frac{\hat{q}_{r}-\hat{\mu}_{Y \mid R}}{\hat{s}_{Y \mid R}} \operatorname{IF}_{i}\left(\hat{s}_{X \mid D}\right) \\
& -\frac{\left(\hat{q}_{r}-\hat{\mu}_{Y \mid R}\right) \hat{s}_{X \mid D}}{\left(\hat{s}_{Y \mid R}\right)^{2}} \operatorname{IF}_{i}\left(\hat{s}_{Y \mid R}\right)+\operatorname{IF}_{i}\left(\hat{\mu}_{X \mid D}\right)
\end{aligned}
$$


where $s$ is either the IQR or the standard deviation. Finally, for a logarithmic location and scale adjustment we have $t\left(q_{r}, \theta\right)=\exp \left(\left(\ln q_{r}-\mu_{\ln (Y) \mid R}\right) \cdot s_{\ln (X) \mid D} / s_{\ln (Y) \mid R}+\mu_{\ln (X) \mid D}\right)$, such that

$$
\begin{aligned}
\operatorname{IF}_{i}\left(\hat{\tilde{q}}_{r}\right)= & \hat{\tilde{q}}_{r}\left(\frac{\hat{s}_{\ln (X) \mid D}}{\hat{q}_{r} \hat{s}_{\ln (Y) \mid R}} \operatorname{IF}_{i}\left(\hat{q}_{r}\right)-\frac{\hat{s}_{\ln (X) \mid D}}{\hat{s}_{\ln (Y) \mid R}} \operatorname{IF}_{i}\left(\hat{\mu}_{\ln (Y) \mid R}\right)+\frac{\ln \hat{q}_{r}-\hat{\mu}_{\ln (Y) \mid R}}{\hat{s}_{\ln (Y) \mid R}} \operatorname{IF}_{i}\left(\hat{s}_{\ln (X) \mid D}\right)\right. \\
& \left.-\frac{\left(\ln \hat{q}_{r}-\hat{\mu}_{\ln (Y) \mid R}\right) \hat{s}_{\ln (X) \mid D}}{\left(\hat{s}_{\ln (Y) \mid R}\right)^{2}} \operatorname{IF}_{i}\left(\hat{s}_{\ln (Y) \mid R}\right)+\operatorname{IF}_{i}\left(\hat{\mu}_{\ln (X) \mid D}\right)\right)
\end{aligned}
$$

In case of a shape adjustment, one of the two distributions is replaced by a location (and, possibly, shape) adjusted variant of the other distribution. The same formulas as above can be applied after choosing the appropriate transformation function and replacing some of the components. For example, if the comparison distribution is shape-and-scale adjusted (and the reference distribution remains unchanged), the relevant transformation functions are $t_{D}(y, \theta)=y-\mu_{X \mid R}+\mu_{X \mid D}$ and $t_{R}(y, \theta)=y$ such that $t\left(q_{r}, \theta\right)=q_{r}-\mu_{X \mid D}+\mu_{X \mid R}$. The main moment condition will be conditional on subsample $\mathcal{R}$ instead of $\mathcal{D}$, meaning that $D_{i}$ and $X_{i}$ in (77) have to be replaced by $R_{i}$ and $Y_{i}$. This further implies that $W_{D}$ in the first term of (76) has to be replaced by $W_{R}$ and that the density in the second term is $\hat{f}_{Y \mid R}\left(\hat{\tilde{q}}_{r}\right)$ instead of $\hat{f}_{X \mid D}\left(\hat{\tilde{q}}_{r}\right)$. If the reference distribution is shape-and-scale adjusted (and the comparison distribution remains unchanged), the transformation function again is $t\left(q_{r}, \theta\right)=q_{r}-\mu_{X \mid D}+\mu_{X \mid R}$, but $\hat{q}_{r}$ is now based on the comparison distribution, that is, $\hat{q}_{r}=\widehat{F}_{X \mid D}^{-1}(r)$, such that the definition of $\operatorname{IF}_{i}\left(\hat{q}_{r}\right)$ in $(78)$ changes.

\subsubsection{Influence function for the relative histogram}

For the influence function of a histogram estimate of the relative density, note that for each bin, the histogram density is equal to the difference between two points on the relative CDF, divided by the bin width. That is

$$
\hat{g}(a, b)=\frac{\widehat{G}(b)-\widehat{G}(a)}{b-a}
$$

The influence function for $\hat{g}(a, b)$ can thus be obtained as

$$
\operatorname{IF}_{i}(\hat{g}(a, b))=\frac{\mathrm{IF}_{i}(\widehat{G}(b))-\mathrm{IF}_{i}(\widehat{G}(a))}{b-a}
$$

\subsubsection{Influence function for the relative PDF}

The relative density estimate for continuous data can be written as

$$
\hat{g}(r)=\frac{1}{W_{D}} \sum_{i \in \mathcal{D}} w_{i} K_{c}\left(r, \hat{r}_{i}, h\right) \quad \text { with } \hat{r}_{i}=\widehat{F}_{Y \mid R}\left(X_{i}\right)
$$


where $K_{c}()$ is a boundary-corrected kernel function as described in section 3.3.1. Note that each individual $\hat{r}_{i}, i \in \mathcal{D}$, has its own moment condition:

$$
\begin{aligned}
h_{i}^{g} & =D_{i}\left(K_{c}\left(r, r_{i}, h\right)-g(r)\right) \\
h_{i}^{r_{j}} & =R_{i}\left(\mathbb{1}\left\{Y_{i} \leq X_{j}\right\}-r_{j}\right) \quad \text { for each } j \in \mathcal{D}
\end{aligned}
$$

This leads to

$$
\begin{aligned}
\operatorname{IF}_{i}(\hat{g}(r)) & =\frac{W}{W_{D}}\left(\hat{h}_{i}^{g}+\sum_{j \in \mathcal{D}} \widehat{\Delta}_{r_{j}}^{g} \operatorname{IF}_{i}\left(\hat{r}_{j}\right)\right) \\
& =\frac{W}{W_{D}}\left(\hat{h}_{i}^{g}+\frac{R_{i}}{W_{R}} \sum_{j \in \mathcal{D}} \delta_{j}\left(\mathbb{1}\left\{Y_{i} \leq X_{j}\right\}-\hat{r}_{j}\right)\right)
\end{aligned}
$$

with $\widehat{\Delta}_{r_{j}}^{g}=\delta_{j} / W$ and $\delta_{j}=w_{j} K_{c}^{\prime}\left(r, \hat{r}_{j}, h\right)$, where $K_{c}^{\prime}\left(r, \hat{r}_{j}, h\right)$ is the derivative of $K_{c}\left(r, r_{j}, h\right)$ with respect to $r_{j}$ at point $\hat{r}_{j}$. The sum in the second part of the equation looks computationally burdensome (complexity $O\left(n_{R} n_{D}\right)$ once we evaluate the IF for all observations), but it can be simplified. Let

$$
\lambda_{i}=\sum_{j \in \mathcal{D}} \delta_{j} \mathbb{1}\left\{Y_{i} \leq X_{j}\right\} \quad \text { and } \quad \Lambda=\sum_{j \in \mathcal{D}} \delta_{j} \hat{r}_{j}
$$

such that

$$
\mathrm{IF}_{i}(\hat{g}(r))=\frac{W}{W_{D}}\left(\hat{h}_{i}^{g}+\frac{R_{i}}{W_{R}}\left(\lambda_{i}-\Lambda\right)\right)
$$

Term $\lambda_{i}$ is equivalent to a "reverse" (summation from the top) and non-normalized CDF of $X$ weighted by $\delta_{j}$ and can be obtained for all observations in a single run across the data. ${ }^{10}$

Location and scale adjustment For the relative PDF based on location (and, possibly, scale) adjusted data, define $\tilde{x}_{i}=t^{-1}\left(X_{i}, \theta\right)$ and replace $r_{i}$ by $\tilde{r}_{i}=F_{Y \mid R}\left(\tilde{x}_{i}\right)$ in the above formulas. Function $t(x, \theta)$ is as defined in section 3.7.2; if $t_{D}(x, \theta)$ is the transformation function applied to the comparison data and $t_{R}(x, \theta)$ is the transformation function applied to the reference data, then

$$
t^{-1}(x, \theta)=t_{R}^{-1}\left(t_{D}(x, \theta), \theta\right)
$$

The adjusted relative PDF can thus be written as

$$
\hat{\tilde{g}}(r)=\frac{1}{W_{D}} \sum_{i \in \mathcal{D}} w_{i} K_{c}\left(r, \hat{\tilde{r}}_{i}, h\right) \quad \text { with } \hat{\tilde{r}}_{i}=\widehat{F}_{Y \mid R}\left(\hat{\tilde{x}}_{i}\right) \text { and } \hat{\tilde{x}}_{i}=t^{-1}\left(X_{i}, \hat{\theta}\right)
$$

\footnotetext{
${ }^{10}$ For the adaptive kernel (see footnote 8 ) a complication arises because the local bandwidth depends on preliminary density estimates. This should only be of secondary importance for the variance estimate so that applying the above equation with $h$ replaced by the relevant local bandwidth (i.e., treating the local bandwidth as fixed) should produce acceptable results in practice.
} 
such that the influence function becomes

$$
\operatorname{IF}_{i}(\hat{\tilde{g}}(r))=\frac{W}{W_{D}}\left(\hat{h}_{i}^{\tilde{g}}+\sum_{j \in \mathcal{D}} \widehat{\Delta}_{\tilde{r}_{j}}^{\tilde{g}} \mathrm{IF}_{i}\left(\hat{\tilde{r}}_{j}\right)\right)
$$

with

$$
\begin{aligned}
\hat{h}_{i}^{\tilde{g}} & =D_{i}\left(K_{c}\left(r, \hat{\tilde{r}}_{i}, h\right)-\hat{\tilde{g}}(r)\right) \\
\widehat{\Delta}_{\tilde{r}_{j}}^{\tilde{g}} & =\delta_{j} / W \quad \text { with } \delta_{j}=w_{j} K_{c}^{\prime}\left(r, \hat{\tilde{r}}_{j}, h\right) \\
\operatorname{IF}_{i}\left(\hat{\tilde{r}}_{j}\right) & =\frac{W}{W_{R}}\left(\hat{h}_{i}^{\tilde{r}_{j}}+\widehat{\Delta}_{\tilde{x}_{j}}^{\tilde{r}_{j}} \mathrm{IF}_{i}\left(\hat{\tilde{x}}_{j}\right)\right) \\
\hat{h}^{\tilde{r}_{j}} & =R_{i}\left(\mathbb{\mathbb { 1 }}\left\{Y_{i} \leq \hat{\tilde{x}}_{j}\right\}-\hat{\tilde{r}}_{j}\right) \\
\widehat{\Delta}_{\tilde{x}_{j}}^{\tilde{r}_{j}} & =\frac{W_{R}}{W} \hat{f}_{Y \mid R}\left(\hat{\tilde{x}}_{j}\right) \\
\operatorname{IF}_{i}\left(\hat{\tilde{x}}_{j}\right) & =\sum_{k=1}^{K} \tau_{j k} \operatorname{IF}_{i}\left(\hat{\theta}_{k}\right) \quad \text { with } \tau_{j k}=\left.\frac{\partial t^{-1}\left(X_{j}, \theta\right)}{\partial \theta_{k}}\right|_{\theta=\hat{\theta}}
\end{aligned}
$$

Similar to above, computational complexity can be reduced by rewriting the influence function as

$$
\operatorname{IF}_{i}(\hat{\tilde{g}}(r))=\frac{W}{W_{D}}\left(\hat{h}_{i}^{\tilde{g}}+\frac{R_{i}}{W_{R}}\left(\lambda_{i}-\Lambda\right)+\sum_{k=1}^{K} \kappa_{k} \mathrm{IF}_{i}\left(\hat{\theta}_{k}\right)\right)
$$

with

$$
\lambda_{i}=\sum_{j \in \mathcal{D}} \delta_{j} \mathbb{1}\left\{Y_{i} \leq \hat{\tilde{x}}_{j}\right\}, \quad \Lambda=\sum_{j \in \mathcal{D}} \delta_{j} \hat{\tilde{r}}_{j}, \quad \kappa_{k}=\frac{1}{W} \sum_{j \in \mathcal{D}} \delta_{j} \hat{f}_{Y \mid R}\left(\hat{\tilde{x}}_{j}\right) \tau_{j k}
$$

In case of a shape adjustment, the same formulas can be used, but various components have to be replaced to take account of the switch in subsamples.

\subsubsection{Influence function for the discrete relative density}

The categorical relative density is defined as

$$
\hat{g}^{k}=\hat{p}_{k}^{D} / \hat{p}_{k}^{R}
$$

with moment conditions

$$
h_{i}^{p_{D}^{k}}=D_{i}\left(\mathbb{1}\left\{X_{i}=k\right\}-p_{k}^{D}\right) \quad \text { and } \quad h_{i}^{p_{R}^{k}}=R_{i}\left(\mathbb{1}\left\{Y_{i}=k\right\}-p_{k}^{R}\right)
$$

where $\hat{p}_{k}^{D}=\widehat{P}_{D}(X=k)$ and $\hat{p}_{k}^{R}=\widehat{P}_{R}(Y=k)$. The influence function can thus be written as

$$
\operatorname{IF}_{i}\left(\hat{g}^{k}\right)=\frac{1}{\hat{p}_{k}^{R}} \mathrm{IF}_{i}\left(\hat{p}_{k}^{D}\right)-\frac{\hat{p}_{k}^{D}}{\left(\hat{p}_{k}^{R}\right)^{2}} \operatorname{IF}_{i}\left(\hat{p}_{k}^{R}\right)
$$

with

$$
\operatorname{IF}_{i}\left(\hat{p}_{k}^{D}\right)=\frac{W}{W_{D}} D_{i}\left(\mathbb{1}\left\{X_{i}=k\right\}-\hat{p}_{k}^{D}\right) \quad \text { and } \quad \operatorname{IF}_{i}\left(\hat{p}_{k}^{R}\right)=\frac{W}{W_{R}} R_{i}\left(\mathbb{1}\left\{Y_{i}=k\right\}-\hat{p}_{k}^{R}\right)
$$




\subsubsection{Influence functions for divergence measures}

Divergence measures are obtained as aggregates of relative density estimates. Hence, their influence functions can be written as aggregates of the influence functions of the density estimates. Assuming the divergence measures are computed form a kernel density estimate on a regular grid or from a histogram density with $K$ evenly sized bins, as described in section 3.4.1, we get

$$
\begin{aligned}
\mathrm{IF}_{i}\left(\widehat{\chi}^{2}\right) & =\frac{2}{K} \sum_{k=1}^{K}\left(\hat{g}_{k}-1\right) \operatorname{IF}_{i}\left(\hat{g}_{k}\right) \\
\mathrm{IF}_{i}(\widehat{\mathrm{KL}}) & =\frac{1}{K} \sum_{k=1}^{K}\left(1+\ln \left(\hat{g}_{k}\right)\right) \operatorname{IF}_{i}\left(\hat{g}_{k}\right) \\
\operatorname{IF}_{i}(\widehat{\mathrm{TVD}}) & =\frac{1}{2 K} \sum_{k=1}^{K} \operatorname{sign}\left(\hat{g}_{k}-1\right) \operatorname{IF}_{i}\left(\hat{g}_{k}\right)
\end{aligned}
$$

where $\hat{g}_{k}$ is the kernel density estimate at evaluation point $r_{k}$ or the histogram estimate for bin $k$. For divergence measures computed from categorical data (see section 3.4.2), the influence functions can be written as

$$
\begin{aligned}
\mathrm{IF}_{i}\left(\widehat{\chi}^{2}\right) & =\sum_{k=1}^{K} 2\left(\frac{\hat{p}_{k}^{D}}{\hat{p}_{k}^{R}}-1\right) \operatorname{IF}_{i}\left(\hat{p}_{k}^{D}\right)+\left(1-\left(\frac{\hat{p}_{k}^{D}}{\hat{p}_{k}^{R}}\right)^{2}\right) \operatorname{IF}_{i}\left(\hat{p}_{k}^{R}\right) \\
\mathrm{IF}_{i}(\widehat{\mathrm{KL}}) & =\sum_{k=1}^{K}\left(1+\ln \left(\frac{\hat{p}_{k}^{D}}{\hat{p}_{k}^{R}}\right)\right) \mathrm{IF}_{i}\left(\hat{p}_{k}^{D}\right)-\frac{\hat{p}_{k}^{D}}{\hat{p}_{k}^{R}} \operatorname{IF}_{i}\left(\hat{p}_{k}^{R}\right) \\
\mathrm{IF}_{i}(\widehat{\mathrm{TVD}}) & =\frac{1}{2} \sum_{k=1}^{K} \operatorname{sign}\left(\hat{p}_{k}^{D}-\hat{p}_{k}^{R}\right)\left(\mathrm{IF}_{i}\left(\hat{p}_{k}^{D}\right)-\mathrm{IF}_{i}\left(\hat{p}_{k}^{R}\right)\right)
\end{aligned}
$$

where $\operatorname{IF}_{i}\left(\hat{p}_{k}^{D}\right)$ and $\operatorname{IF}_{i}\left(\hat{p}_{k}^{R}\right)$ are as defined in section 3.7.5.

\subsubsection{Influence functions for polarization indices}

The MRP can be written as

$$
\mathrm{MRP}=\frac{1}{W_{D}} \sum_{i \in \mathcal{D}} w_{i}\left(4\left|\hat{\tilde{r}}_{i}-0.5\right|-1\right) \quad \text { with } \hat{\tilde{r}}_{i}=\widehat{F}_{Y \mid R}\left(\hat{\tilde{x}}_{i}\right) \text { and } \hat{\tilde{x}}_{i}=t^{-1}\left(X_{i}, \hat{\theta}\right)
$$

where $t^{-1}(x, \theta)$ is as defined in (90). We see that the MRP has the same structure as an estimate of the relative PDF based on location (and, possibly, scale) adjusted data. We can thus obtain the influence function using (99) with $h_{i}^{\tilde{g}}$ replaced by

$$
h_{i}^{\mathrm{MRP}}=D_{i}\left(\left(4\left|\tilde{r}_{i}-0.5\right|-1\right)-\mathrm{MRP}\right)
$$


and $\delta_{j}$ set to

$$
\delta_{j}=w_{j} 4 \operatorname{sign}\left(\hat{\tilde{r}}_{j}-0.5\right)
$$

Likewise, the influence function for the LRP can be obtained by using

$$
h_{i}^{\mathrm{LRP}}=D_{i}\left(\left(8\left|\tilde{r}_{i}-0.5\right| \mathbb{1}\left\{\tilde{r}_{i}<.5\right\}-1\right)-\mathrm{LRP}\right)
$$

and

$$
\delta_{j}=w_{j} 8 \operatorname{sign}\left(\hat{\tilde{r}}_{j}-0.5\right) \mathbb{1}\left\{\hat{\tilde{r}}_{j}<.5\right\}
$$

Note that $\mathbb{1}\left\{\tilde{r}_{i}<.5\right\}$ always selects half of the comparison data because the data has been median adjusted. Assuming it fixed should not introduce significant bias into the variance estimates. The influence function for the URP can be derived analogously.

\subsubsection{Influence functions for descriptive statistics}

Like the MRP, summary statistics of the relative ranks such as the mean or the standard deviation have a structure that is very similar to the relative PDF. For the mean $\hat{\mu}$ of the (possibly adjusted) relative ranks, the influence function can be obtained by replacing $h_{i}^{\tilde{g}}$ in equation (99) by

$$
h_{i}^{\mu}=D_{i}\left(\tilde{r}_{i}-\mu\right)
$$

and setting $\delta_{j}$ to

$$
\delta_{j}=w_{j}
$$

Likewise, for the variance $\hat{\sigma}^{2}$ of the relative ranks we can use

$$
h_{i}^{\sigma^{2}}=D_{i}\left(\left(\tilde{r}_{i}-\mu\right)^{2}-\sigma^{2}\right) \quad \text { and } \quad \delta_{j}=2 w_{j}\left(\hat{\tilde{r}}_{j}-\hat{\mu}\right)
$$

The influence function for the standard deviation $\hat{\sigma}$ is given as:

$$
\operatorname{IF}_{i}(\hat{\sigma})=\frac{1}{2 \hat{\sigma}} \operatorname{IF}_{i}\left(\hat{\sigma}^{2}\right)
$$

For quantile $\hat{q}(p)$ of the relative ranks it is easier to follow a different approach. Note that the quantile can be written as

$$
q(p)=G^{-1}(p)=F_{Y \mid R}\left(F_{X \mid D}^{-1}(p)\right)
$$

That is, a quantile of the relative ranks of $F_{X \mid D}$ with respect to $F_{Y \mid R}$ is equivalent to a point on the relative CDF of $F_{Y \mid R}$ with respect to $F_{X \mid D}$. We can thus obtain the influence function as in section 3.7.2, but with swapped distributions. Finally, the influence function for the interquartile range is given as:

$$
\operatorname{IF}_{i}(\mathrm{IQR})=\operatorname{IF}_{i}(\hat{q}(0.75))-\operatorname{IF}_{i}(\hat{q}(0.25))
$$




\subsubsection{Influence functions in case of covariate balancing}

If covariates are balanced using the reweighting approach, the influence functions need to be adjusted to take account of the fact that the balancing weights have been estimated. I will discuss two reweighting methods below: IPW based on logistic regression and entropy balancing. Deriving the influence functions is relatively easy in these cases as the weights are obtained from a parametric model. Non-parametric reweighting methods such as matching are more challenging; I leave it to future research to work out the details for such methods.

Given covariates $Z$, logit IPW and entropy balancing both estimate a vector of coefficients $\gamma$ from which the balancing weights $\widetilde{w}$ are computed. To obtain the influence function for a reweighted relative distribution statistic, we could follow the procedures outlined above, but additionally incorporate $\gamma$ by apply equation (63) to each of the parameters within the definition of the statistic that depend on the balancing weights. However, working though the math shows that this can be simplified. Let $\theta$ be a covariate-adjusted relative distribution statistic and $\operatorname{let}_{\mathrm{IF}_{i}^{*}}(\hat{\theta})$ be a provisional influence function that assumes the balancing weights as fixed (the provisional influence function is obtained in the same way as without covariateadjustment, only that the base weights $w$ are replaced by the balancing weights $\widetilde{w}$ ). The final influence function that no longer assumes the balancing weights as fixed can then be computed as

$$
\operatorname{IF}_{i}(\hat{\theta})=\frac{\widetilde{w}_{i}}{w_{i}} \operatorname{IF}_{i}^{*}(\hat{\theta})+\hat{\Delta}_{\gamma}^{\mathrm{IF}^{*}} \operatorname{IF}_{i}(\hat{\gamma})
$$

For both logit IPW and entropy balancing the vector of partial derivatives of $\operatorname{IF}_{i}^{*}(\hat{\theta})$ by $\gamma$ can be written as

$$
\frac{\partial \mathrm{IF}_{i}^{*}(\hat{\theta})}{\partial \gamma}=\frac{\widetilde{w}_{i}}{w_{i}}\left(1-T_{i}\right) \mathrm{IF}_{i}^{*}(\hat{\theta}) Z_{i}
$$

such that

$$
\hat{\Delta}_{\gamma}^{\mathrm{IF}}=\frac{1}{W} \sum_{i=1}^{N} \widetilde{w}_{i}\left(1-T_{i}\right) \mathrm{IF}_{i}^{*}(\hat{\theta}) Z_{i}
$$

where $T$ is an indicator for the "treatment" group $(T=R$ if the comparison group is reweighted, $T=D$ if the reference group is reweighted; the two groups are assumed distinct and exhaustive). The definition of the balancing weights and the expression for $\operatorname{IF}_{i}(\hat{\gamma})$ differ between the two procedures, as is described next.

Logit IPW In case of logit IPW the balancing weights are defined as

$$
\widetilde{w}_{i}=\left\{\begin{array}{ll}
w_{i} \frac{\hat{p}_{i}}{1-\hat{p}_{i}} c & \text { if } T_{i}=0 \\
w_{i} & \text { if } T_{i}=1
\end{array} \quad \text { with } \quad \hat{p}_{i}=\frac{e^{Z_{i} \hat{\gamma}}}{1+e^{Z_{i} \hat{\gamma}}}\right.
$$

where $Z_{i}$ is a vector of covariates (typically including a constant), $\hat{\gamma}$ is the corresponding coefficient vector estimated by logistic regression, and $c$ is a scaling factor ensuring that the 
sum of weights remains constant (see section 3.6; $c$ can be treated as fixed). As discussed in Jann (2020), the logistic regression moment conditions for $\gamma$ can be written as

$$
h_{i}^{\gamma}=Z_{i}^{\prime}\left(T_{i}-p_{i}\right)
$$

such that

$$
\operatorname{IF}_{i}(\hat{\gamma})=\left(-\hat{\Delta}^{\gamma}\right)^{-1} Z_{i}^{\prime}\left(T_{i}-\hat{p}_{i}\right) \quad \text { with } \quad \hat{\Delta}^{\gamma}=\frac{1}{W} \sum_{i=1}^{N} w_{i} Z_{i}^{\prime} \hat{p}_{i}\left(1-\hat{p}_{i}\right) Z_{i}
$$

Entropy balancing For entropy balancing, the weights are given as

$$
\widetilde{w}_{i}= \begin{cases}w_{i} e^{z_{i} \hat{\beta}+\hat{\alpha}} c & \text { if } T_{i}=0 \\ w_{i} & \text { if } T_{i}=1\end{cases}
$$

where $z_{i}$ is a vector of $k$ covariates without constant, and $\hat{\beta}$ and $\hat{\alpha}$ are the entropy balancing coefficients. The estimation of $\beta$ involves a vector of auxiliary parameters $\mu$, the means of $Z$ in the treatment group. Furthermore, $\alpha$ involves auxiliary parameter $p$, the treatment probability. Based on Jann (2020) the entropy balancing moment conditions can be written as

$$
\begin{aligned}
h_{i}^{\beta} & =\left(1-T_{i}\right) \frac{\widetilde{w}_{i}}{w_{i} c}\left(z_{i}^{\prime}-\mu\right) & h_{i}^{\alpha} & =\left(1-T_{i}\right)\left(\frac{\widetilde{w}_{i}}{w_{i} c}-\frac{p}{1-p}\right) \\
h_{i}^{\mu} & =T_{i}\left(z_{i}^{\prime}-\mu\right) & h_{i}^{p} & =T_{i}-p
\end{aligned}
$$

such that

$$
\operatorname{IF}_{i}(\hat{\beta})=\left(-\hat{\Delta}^{\beta}\right)^{-1}\left(\hat{h}_{i}^{\beta}+\hat{\Delta}_{\mu}^{\beta}\left(-\hat{\Delta}^{\mu}\right)^{-1} \hat{h}_{i}^{\mu}\right)=\left(-\hat{\Delta}^{\beta}\right)^{-1}\left(\hat{h}_{i}^{\beta}-\hat{h}_{i}^{\mu}\right)
$$

with

$$
\hat{\Delta}^{\beta}=\frac{1}{W} \sum_{i=1}^{N} w_{i} \hat{h}_{i}^{\beta} z_{i}=\frac{1}{W} \sum_{i=1}^{N} \frac{\widetilde{w}_{i}}{c}\left(1-T_{i}\right)\left(z_{i}^{\prime}-\hat{\mu}\right) z_{i}
$$

The simplification on the right in (129) follows from

$$
\begin{aligned}
& \hat{\Delta}^{\mu}=\frac{1}{W} \sum_{i=1}^{N}-w_{i} T_{i} \operatorname{diag}_{k}(1)=-\operatorname{diag}_{k}\left(W_{T}\right) / W \\
& \hat{\Delta}_{\mu}^{\beta}=\frac{1}{W} \sum_{i=1}^{N}-\frac{\widetilde{w}_{i}}{c}\left(1-T_{i}\right) \operatorname{diag}_{k}(1)=-\operatorname{diag}_{k}(\Omega) / W
\end{aligned}
$$

where $\Omega=\sum_{i: T_{i}=0} \widetilde{w}_{i} / c$, because moment condition $h^{\alpha}$ ensures that $\Omega=W_{T}$. Furthermore, for $\alpha$ we get

$$
\begin{aligned}
\operatorname{IF}_{i}(\hat{\alpha}) & =\frac{1}{-\hat{\Delta}^{\alpha}}\left(\hat{h}_{i}^{\alpha}+\hat{\Delta}_{p}^{\alpha} \frac{1}{-\hat{\Delta}^{p}} \hat{h}_{i}^{p}+\hat{\Delta}_{\beta}^{\alpha} \operatorname{IF}_{i}(\hat{\beta})\right) \\
& =-\frac{\Omega}{W}\left(\hat{h}_{i}^{\alpha}-\frac{T_{i}-\hat{p}}{1-\hat{p}}+\hat{\Delta}_{\beta}^{\alpha} \operatorname{IF}_{i}(\hat{\beta})\right) \quad \text { with } \quad \hat{\Delta}_{\beta}^{\alpha}=\frac{1}{W} \sum_{i=1}^{N} \frac{\widetilde{w}_{i}}{c} z_{i}
\end{aligned}
$$


Finally, let

$$
Z_{i}=\left(z_{i}, 1\right) \quad \hat{\gamma}=\left(\hat{\beta}^{\prime}, \hat{\alpha}\right)^{\prime} \quad \operatorname{IF}_{i}(\hat{\gamma})=\left(\operatorname{IF}_{i}(\hat{\beta})^{\prime}, \operatorname{IF}_{i}(\hat{\alpha})\right)^{\prime}
$$

so that the results have the same format as for the logit IPW.

\section{The reldist command}

Stata command reldist implements the methods discussed above. The moremata (Jann, 2005) package is required. For installation, type

- ssc install reldist, replace

- ssc install moremata, replace

or

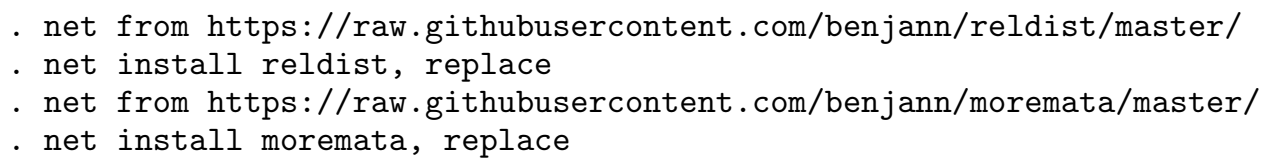

The versions at GitHub might be updated more frequently than the versions at the SSC Archive.

\subsection{Syntax}

\section{Estimation}

Command reldist has two syntaxes. Use Syntax 1 if you want to analyze the relative distribution of a single variable between two groups or subpopulations. Syntax 2 is for comparing two variables within a single sample.

Syntax 1 (two-sample relative distribution):

reldist subcmd varname $[i f][i n]$ [weight], by(groupvar) [ options ]

where groupvar identifies two groups to be compared.

Syntax 2 (paired relative distribution):

reldist subcmd varname refvar [if $]$ [in] [weight] [, options ]

where varname and refvar specify two variables to be compared.

In both cases, fweights, pweights, and iweights are allowed (see [u] 11.1.6 weight) and subcmd can be 
pdf to estimate the density function of the relative distribution, possibly including a histogram of the relative density,

histogram to estimate a histogram of the relative density,

cdf to estimate the relative distribution function (equivalent to a so-called probability-probability plot),

divergence to estimate the Kullback-Leibler divergence (entropy), the Chi-squared divergence, or the dissimilarity index (total variation distance) of the relative distribution,

mrp to estimate the median relative polarization index (MRP), as well as its decomposition into a lower and and upper polarization index (LRP and URP),

summarize to estimate summary statistics such as the mean or the median of the relative ranks and, optionally, store the relative ranks in a new variable.

\section{Creating a graph after estimation}

After applying reldist pdf, reldist hist, or reldist cdf, command reldist graph can be used to draw a graph of the results. The syntax is

reldist graph [, graph_options ]

An alternative is generate the graph directly using option graph() with reldist pdf, reldist hist, or reldist cdf.

\section{Storing influence functions after estimation}

Command predict can be applied after reldist to generate the influence functions of the estimated parameters (one variable per parameter). The syntax is:

predict $\{$ stub* $\mid$ newvarlist $\}[$ if $][$ in $]$ [, scores density_options $]$

where stub specifies a common prefix for the names of the generated variables or, alternatively, newvarlist specifies an explicit list of variable names to be used. Option scores is allowed for compatibility reasons; it does not do anything. density_options can be used to modify how auxiliary densities are estimated during the computation of the influence functions; see page 41 for a description of available density_options (option boundary() will have no effect, as unbounded support is assumed for auxiliary densities).

Command total (see $[\mathrm{R}]$ total) can be applied to the stored influence functions to replicate the standard errors reported by reldist. 


\subsection{Options for reldist}

\section{Main options}

by (groupvar) specifies a binary variable that identifies the two groups to be compared. By default, the group with the lower value will be used as the reference group. by() is required in Syntax 1 and not allowed in Syntax 2.

swap reverses the order of the groups identified by by (). swap is only allowed in Syntax 1 .

pooled uses the pooled distribution across both groups as reference distribution. pooled is only allowed in Syntax 1.

balance (spec) balances covariate distributions between the comparison group and the reference group using reweighting. balance() is only allowed in Syntax 1. The syntax of spec is

[method: ] varlist [, options $]$

where method is either ipw for inverse probability weighting based on logistic regression (the default) or eb for entropy balancing (using mm_ebal() from moremata), varlist specifies the list of covariates to be balanced, and options are as follows:

reference reweights the reference group. The default is to reweight the comparison group. Option pooled is not allowed with balance(, reference).

contrast compares the balanced distribution to the unbalanced distribution. Use this option to see how the balancing changes the distribution. If contrast is specified together with reference, the balanced reference distribution will be used as the comparison distribution. If contrast is specified without reference, the balanced comparison distribution will be used as the reference distribution.

logit_options are options to be passed through to $[\mathrm{R}]$ logit. logit_options are only allowed if method is ipw.

btolerance (\#), with \# $\geq 0$, specifies the tolerance for the entropy balancing algorithm. The default is btolerance(1e-5). A warning message is displayed if a balancing solution is not within the specified tolerance. btolerance() is only allowed if method is eb.

noisily displays the output of the balancing procedure.

generate (newvar) stores the balancing weights in variable newvar. This is useful if you want to check whether covariates have been successfully balanced.

adjust (spec) applies location, scale, and shape adjustments to the comparison and reference distributions. adjust() is not allowed with reldist mrp. The syntax of spec is

$$
\text { adjust [, options ] }
$$

where adjust specifies the desired adjustments. adjust may contain any combination of at most two of the following keywords: 
location adjust location

scale adjust scale

shape adjust shape

By default, the specified adjustments are applied to the comparison distribution. However, a colon may be included in adjust to distinguish between distributions: Keywords before the colon affect the comparison distribution; keywords after the colon affect the reference distribution. For example, type adjust (location scale) to adjust the location and scale of the comparison distribution. Likewise, you could type adjust (:location scale) to adjust the reference distribution. Furthermore, adjust(location: shape) would adjust the location of the comparison distribution and the shape of the reference distribution. options are as follows:

mean uses the mean for the location adjustment. The default is to use the median.

sd uses the standard deviation for the scale adjustment. The default is to use the IQR (interquartile range).

multiplicative uses a multiplicative adjustment instead of an additive adjustment. adjust may only contain one keyword in this case, either location or shape. Error will be returned if the location ratio between the comparison distribution and the reference distribution is not strictly positive.

logarithmic performs the adjustments on logarithmically transformed data. Error will be returned if the data is not strictly positive.

rank_options specify the details about the computation of relative ranks. These options are irrelevant for reldist histogram, reldist cdf, reldist divergence unless option pdf is specified, and for reldist pdf if discrete or categorical is specified. The options are as follows:

nobreak changes how the relative ranks are computed in case of ties. By default, reldist breaks ties randomly for comparison values that have ties in the reference distribution (in ascending order of weights, if weights have been specified). This leads to improved results if there is heaping in the data. Specify nobreak to omit breaking ties.

nomid changes how the relative ranks are computed in case of ties. By default, reldist uses midpoints of the steps in the cumulative distribution for comparison values that have ties in the reference distribution. This ensures that the average relative rank is equal to 0.5 if the comparison and reference distributions are identical. Specify nomid to assign relative ranks based on full steps in the CDF.

descending sorts tied observations in descending order of weights. The default is to use ascending sort order. Option descending has no effect if nobreak is specified or if there are no weights.

nostable breaks ties randomly (within unique values of weights). The default is to break the ties in the sort order of the data (within unique values of weights). Option nostable has no effect on the results reported by reldist. It may, however, affect 
the ranks stored by option generate() or the influence functions stored by predict (unless option nobreak is specified).

replace allows replacing existing variables. This is relevant for generate() with reldist summarize and generate() in balance().

\section{Additional options for reldist pdf}

$\mathrm{n}$ (\#) sets the number of evaluation points for which the PDF is to be computed. A regular grid of \# evaluation points between 0 and 1 will be used. The default is $\mathrm{n}(101)$ (unless option discrete or categorical is specified, in which case $\mathrm{n}($ ) has no default). Only one of $\mathrm{n}()$, at (), and $\operatorname{atx}()$ is allowed.

at (\{numlist $\mid$ matname $\})$ specifies a custom grid of evaluation points between 0 and 1 , either by providing a numlist (see [U] 11.1.8 numlist) or the name of a matrix containing the values (the values will be taken from the first row or the first column of the matrix, depending on which is larger). Only one of $\mathrm{n}()$, at (), and atx () is allowed.

atx $[(\{$ comparison $\mid$ reference $\mid$ numlist $\mid$ matname $\})]$, specified without argument, causes the relative PDF to be evaluated at each distinct outcome value that exists in the data (possibly after applying adjust()), instead of using a regular evaluation grid on the probability scale. All outcome values across both distributions will be considered. To restrict the evaluation points to outcome values from the comparison distribution or from the reference distribution, specify atx(comparison) or atx(reference), respectively. Alternatively, specify a grid of custom values, either by providing a numlist (see [u] 11.1.8 numlist) or the name of a matrix containing the values (the values will be taken from the first row or the first column of the matrix, depending on which is larger). Only one of $\mathrm{n}()$, at (), and $\operatorname{atx}()$ is allowed.

discrete causes the data to be treated as discrete. The relative PDF will then be evaluated at each level of the data as the ratio of the level's frequency between the comparison distribution and the reference distribution instead of using kernel density estimation, and the result will be displayed as a step function. If option $\mathrm{n}()$ or at () is specified, the step function will be evaluated at the points of the corresponding probability grid instead of returning the relative density for each outcome level. Options nobreak, nomid, descending, and density_options have no effect if discrete is specified. Furthermore, options histogram() and adjust() are not allowed.

categorical is like discrete, but additionally requests that the data only contains positive integers. Factor-variable notation will be used to label the coefficient in the output table.

histogram $[(\#)]$ computes a histogram in addition to the PDF, where \# is the number of bins. If \# is omitted, 10 bins will be used.

alt uses an alternative estimation method for the histogram. See the histogram options below. 
density_options set the details of kernel density estimation. The options are as follows:

bwidth $(\{\# \mid \operatorname{method}[$, nord $]\})$ determines the bandwidth of the kernel, the halfwidth of the estimation window around each evaluation point. Use bwidth(\#), \#>0, to set the bandwidth to a specific value. Alternatively, type bwidth(method) to choose an automatic bandwidth selection method. Choices are silverman (optimal of Silverman), normalscale (normal scale rule), oversmoothed (oversmoothed rule), sjpi (Sheather-Jones solve-the-equation plug-in), dpi[(\#)] (Sheather-Jones direct plug-in estimate, where \# specifies the number of stages of functional estimation; default is 2), or isj (diffusion estimator bandwidth). The default is bw (sjpi). See Jann (2007) for information on silverman, normalscale, oversmoothed, sjpi, and dpi. For isj, see Botev et al. (2010).

By default, if estimating the density of the relative data, all bandwidth selectors include a correction for relative data based on Ćwik and Mielniczuk (1993). Specify suboption nord to omit the correction.

bwadjust (\#) multiplies the bandwidth by \#, where \# $>0$. Default is bwadjust(1).

boundary (method) sets the type of boundary correction method. Choices are renorm (renormalization method; the default), reflect (reflection method), or lc (linear combination technique). See Jann (2007) for details on boundary correction methods.

adaptive(\#) specifies the number of iterations used by the adaptive kernel density estimator. The default is adaptive(0) (non-adaptive density estimator).

kernel (kernel) specifies the kernel function to be used. kernel may be epanechnikov (Epanechnikov kernel function), epan2 (alternative Epanechnikov kernel function), biweight (biweight kernel function), triweight (triweight kernel function), cosine (cosine trace), gaussian (Gaussian kernel function), parzen (Parzen kernel function), rectangle (rectangle kernel function) or triangle (triangle kernel function). The default is kernel (gaussian).

napprox (\#) specifies the grid size used by the binned approximation density estimator (and by the data-driven bandwidth selectors). The default is napprox(512).

exact causes the exact kernel density estimator to be used instead of the binned approximation estimator. The exact estimator can be slow in large datasets if the density is to be evaluated at many points.

graph[(graph_options)] displays the results in a graph. The coefficients table will be suppressed in this case (unless option table is specified). Alternatively, use command reldist graph to display the graph after estimation.

$\operatorname{ogrid}(\#)$ sets the size of the approximation grid for outcome labels. The default is ogrid(401). The grid is stored in e(ogrid) and will be used by graph option olabel() to determine the positions of outcome labels. Type noogrid to omit the computation of the grid (no outcome labels will then be available for the graph). Option ogrid() is only allowed if the relative density is computed with respect an evaluation grid on the 
probability scale. If the relative density is evaluated with respect to specific outcome values (e.g. if atx () is specified), the outcome labels will be obtained from the information stored in e(at).

\section{Additional options for reldist histogram}

$\mathrm{n}(\#)$ specifies the number of histogram bars. The reference distribution will be divided into \# bins of equal width. That is, each bin will cover 1/\#th of the reference distribution. The default is $\mathrm{n}(10)$.

alt uses an alternative estimation method. The default method obtains the relative histogram by computing the empirical CDF of both distributions at all values that exist in the data (across both distributions). The alternative method obtains the relative histogram based on the empirical CDF of the relative ranks. In both cases, if necessary, linear interpolation will be used to map the relative CDF to the evaluation points.

discrete causes the data to be treated as discrete. The relative density will then be evaluated at each level of the data as the ratio of the level's frequency between the two distributions and the width of bars will be proportional to the reference distribution. Option alt has no effect and options $n()$ and adjust() are not allowed if discrete is specified.

categorical is like discrete, but additionally requests that the data only contains positive integers. Factor-variable notation will be used to label the coefficient in the output table.

graph $[($ graph_options $)]$ displays the results in a graph. The coefficients table will be suppressed in this case (unless option table is specified). Alternatively, use command reldist graph to display the graph after estimation.

ogrid(\#) sets the size of the approximation grid for outcome labels. The default is ogrid(401). The grid is stored in e(ogrid) and will be used by graph option olabel() to determine the positions of outcome labels. Type noogrid to omit the computation of the grid (no outcome labels will then be available for the graph). ogrid() is not allowed together with discrete or categorical.

\section{Additional options for reldist cdf}

$\mathrm{n}$ (\#) sets the number of evaluation points for which the CDF is to be computed. A regular grid of \# evaluation points between 0 and 1 will be used. The default is $n(101)$ (unless option discrete or categorical is specified, in which case $\mathrm{n}()$ has no default). Only one of $\mathrm{n}()$, at (), and $\operatorname{atx}()$ is allowed.

at ( $\{$ numlist $\mid$ matname $\})$ specifies a custom grid of evaluation points between 0 and 1 , either by providing a numlist (see [u] 11.1.8 numlist) or the name of a matrix containing the values (the values will be taken from the first row or the first column of the matrix, depending on which is larger). Only one of $\mathrm{n}()$, at (), and atx () is allowed. 


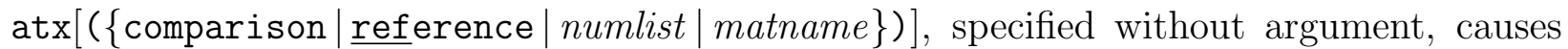
the relative CDF to be evaluated at each distinct outcome value that exists in the data (possibly after applying adjust()), instead of using a regular evaluation grid on the probability scale. All outcome values across both distributions will be considered. To restrict the evaluation points to outcome values from the comparison distribution or from the reference distribution, specify atx(comparison) or atx(reference), respectively. Alternatively, specify a grid of custom values, either by providing a numlist (see [U] 11.1.8 numlist) or the name of a matrix containing the values (the values will be taken from the first row or the first column of the matrix, depending on which is larger). Only one of $\mathrm{n}()$, at (), and $\operatorname{atx}()$ is allowed.

alt uses an alternative estimation method. The default method obtains the relative CDF by computing the empirical CDF of both distributions at all values that exist in the data (across both distributions). The alternative method obtains the relative CDF based on the empirical CDF of the relative ranks. In both cases, if necessary, linear interpolation will be used to map the relative CDF to the evaluation points.

discrete causes the data to be treated as discrete. The relative CDF will then be evaluated at each observed outcome value instead of using an evaluation grid on the probability scale. Option discrete leads to the same result as specifying atx. Option adjust() is not allowed if discrete is specified.

categorical is like discrete, but additionally requests that the data only contains positive integers. Factor-variable notation will be used to label the coefficient in the output table.

graph[(graph_options)] displays the results in a graph. The coefficients table will be suppressed in this case (unless option table is specified). Alternatively, use command reldist graph to display the graph after estimation.

ogrid(\#) sets the size of the approximation grid for outcome labels. The default is ogrid(401). The grid is stored in e(ogrid) and will be used by graph option olabel() to determine the positions of outcome labels. Type noogrid to omit the computation of the grid (no outcome labels will then be available for the graph). Option ogrid() is only allowed if the relative CDF is computed with respect an evaluation grid on the probability scale. If the relative $\mathrm{CDF}$ is evaluated with respect to specific outcome values (e.g. if $\operatorname{atx}()$ is specified), the outcome labels will be obtained from the information stored in e (at).

\section{Additional options for reldist divergence}

over (overvar) computes results for each subpopulation defined by the values of overvar.

entropy or $\mathrm{kl}$ computes the Kullback-Leibler divergence (entropy) of the relative distribution. This is the default.

chi2 or chisquared computes the Chi-squared divergence of the relative distribution. 
tvd or dissimilarity computes the dissimilarity index (total variation distance) of the relative distribution.

all computes all supported divergence measures. all is equivalent to entropy chi2 tvd.

$\mathrm{n}$ (\#) specifies the number of histogram bars or, if option pdf is specified, the number of kernel density evaluation points used to estimate the relative distribution. The default is $n(20)$ or, if option pdf is specified, $n(100)$.

alt uses an alternative estimation method for the histogram. See the histogram options above.

pdf computes the divergence measures based on a kernel density estimate instead of a histogram estimate.

density_options set the details of the the kernel density estimation. This is only relevant if option pdf is specified. See page 41 for available options.

discrete causes the data to be treated as discrete. The relative density will then be evaluated at each level of the data as the ratio of the level's frequency between the two distributions. Option alt has no effect and options $n()$, pdf, and adjust() are not allowed if discrete is specified.

categorical is like discrete, but additionally requests that the data only contains positive integers.

compare $[$ (options) $]$ estimates divergence measures for two models of the relative distribution, a main model and an alternative model, and also reports the difference between the two variants. options are balance() and adjust() as described above. balance() and adjust() specified as main options are applied to the main model; balance() and adjust() specified within compare() are applied to the alternative model.

\section{Additional options for reldist mrp}

over(overvar) computes results for each subpopulation defined by the values of overvar.

multiplicative applies multiplicative location adjustment. The default is to use additive adjustment. Only one of logarithmic and multiplicative is allowed.

logarithmic causes the location (and, optionally, scale) adjustment to be performed on the logarithmic scale. Only one of logarithmic and multiplicative is allowed.

$\underline{\text { scale }}[(\mathrm{sd})]$ adjusts the scale of the data before computing the polarization indices. If scale is specified without argument, the IQR (interquartile range) is used; that is, the scale of the data will be adjusted such that the IQR is the same in both distributions. Specify scale(sd) to use the standard deviation instead of the IQR. scale() is not allowed if multiplicative is specified. 


\section{Additional options for reldist summarize}

over (overvar) computes results for each subpopulation defined by the values of overvar.

statistics (statnames) specifies a space separated list of summary statistics to be reported.

The default is statistics(mean). The following summary statistics are supported:

$\begin{array}{ll}\text { mean } & \text { mean } \\ \text { variance } & \text { variance } \\ \text { sd } & \text { standard deviation } \\ \text { median } & \text { median; equivalent to p50 } \\ \text { p\# } & \text { \#th percentile, where \# is an integer between } 1 \text { and } 99 \\ \text { iqr } & \text { interquartile range (p75 - p25) } \\ \text { te (newvar) } & \text { stores the relative ranks (based on adjusted data) in variable newvar } . \\ \text { ending on adjust (), different observations may be filled in. }\end{array}$

\section{Variance estimation options}

level (\#) specifies the confidence level, as a percentage, for confidence intervals. The default is level(95) or as set by set level (see [R] level).

vce (vcetype) determines how standard errors and confidence intervals are computed. vcetype may be:

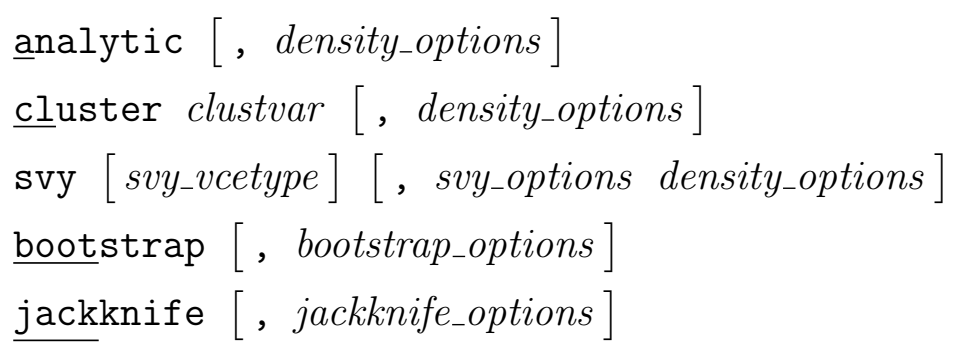

The default is vce (analytic), which computes the standard errors based on influence functions. Likewise, vce(cluster clustvar) computes influence-function based standard errors allowing for intragroup correlation, where clustvar specifies to which group each observation belongs. In both cases, density_options specify how auxiliary densities are estimated during the computation of the influence functions (see page 41 for details; option boundary() will have no effect, as unbounded support is assumed for auxiliary densities).

vce (svy) computes standard errors taking the survey design as set by [svy] svyset into account. The syntax is equivalent to the syntax of the svy prefix command (see [svy] svy); that is, vce(svy) is reldist's way to support the svy prefix. If svy_vcetype is set to linearized, the standard errors are estimated based on influence functions; use density_options to specify the details of auxiliary density estimation in this case. For svy_vcetype other than linearized, density_options are not allowed. 
vce (bootstrap) and vce (jackknife) compute standard errors using [R] bootstrap or $[\mathrm{R}]$ jackknife, respectively; see $[\mathrm{R}]$ vce_option.

If a replication technique is used for standard error estimation (i.e. vce(bootstrap), vce(jackknife), vce(svy) with svy_vcetype other than linearized), the bandwidth used by reldist pdf will be held fixed across replications (that is, if relevant, the bandwidth will be determined upfront and then held constant). If you want to repeat bandwidth search in each replication, use bootstrap, jackknife, or svy as a prefix command.

Simulation results suggest that the influence-function based standard errors work well in most situations. They may be severely biased, however, if there is heaping in the data. Replication-based techniques may yield more valid results in this case.

nose prevents reldist from computing standard errors. This saves computer time.

\section{Reporting options}

citransform reports transformed confidence intervals depending on the type of the reported statistic (log transform for PDF and histogram density, logit transform for CDF and descriptive statistics, inverse hyperbolic tangent transform for polarization indices). citransform only has an effect in Stata 15 or newer.

noheader suppress the output header.

notable suppresses the output table containing the estimated coefficients. table enforces displaying the table if option graph() has been specified.

display_options are standard reporting options such as cformat() or coeflegend; see the reporting options in $[\mathrm{R}]$ Estimation options.

\subsection{Options for reldist graph}

\section{Main graph options}

refline(line_options) specifies options to affect the rendition of the parity line. See help [G] line_options.

norefline suppresses the parity line.

\section{Additional options after reldist pdf}

cline_options affect the rendition of the PDF line. See help [G] cline_options.

histopts (options) specifies options to affect the rendition of the histogram bars (if a histogram was computed) and the corresponding confidence spikes. options are as follows: 
barlook_options affect the rendition of the histogram bars. See help [G] help barlook_options.

ciopts (rcap_options) specifies options to affect the rendition of the confidence spikes of the histogram bars. See help [G] rcap_options.

noci omits the confidence spikes of the histogram bars.

nohistogram omits the histogram bars.

\section{Additional options after reldist histogram}

barlook_options affect the rendition of the histogram bars. See help [G] help barlook_options.

\section{Additional options after reldist cdf}

noorigin prevents adding a $(0,0)$ coordinate to the plotted line. If the first $X$ coordinate of the CDF is larger than zero and the range of the CDF has not been restricted by at () or atx () , reldist graph will automatically add a $(0,0)$ coordinate to the plot. Type noorigin to override this behavior.

cline_options affect the rendition of the CDF line. See help [G] cline_options.

\section{Confidence intervals}

level(\#) specifies the confidence level, as a percentage, for confidence intervals.

citransform plots transformed confidence intervals depending on the type of the reported statistic (log transform for PDF and histogram density, logit transform for CDF).

ci (name) obtains the confidence intervals from e (name) instead of computing them from e (V). e (name) must contain two rows and the same number of columns as e(b). For example, after bootstrap estimation, you could type ci (ci_percentile) to plot percentile confidence intervals. ci() and level() are not both allowed.

ciopts (options) specifies options to affect the rendition of the confidence intervals. See help [G] area_options or, after, reldist histogram help [G] rcap_options. Use option recast() to change the plot type used for confidence intervals. For example, type ciopts (recast(rline)) to use two lines instead of an area.

noci omits the confidence intervals. 


\section{Outcome labels}

$[\mathrm{y}]$ olabel $[($ spec $)]$ adds outcome labels on a secondary axis. olabel() adds outcome labels for the reference distribution; yolabel() adds outcome labels for the comparison distribution (only allowed after reldist cdf). The syntax of spec is

$$
\text { [\#\# | numlist }][,\{\text { noprune } \mid \text { prune(mindist) }\} \text { at format (\%fmt) suboptions }]
$$

\#\# requests that (approximately) \# outcome labels be added at (approximately) evenlyspaced positions; the default is \#6. Alternatively, specify numlist to generate labels for given outcome values.

prune (mindist) requests that an outcome label (but not its tick) is to be omitted if its distance to the preceding label is less than mindist (an exception are labels that have the same position; in such a case the largest label will be printed). The default is prune (0.1); type prune (0) or noprune to print labels at all positions. The difference between prune (0) and noprune is that prune (0) will only print one label per position whereas noprune prints all labels, including labels that have the same position.

at causes numlist to be interpreted as a list of probabilities for which outcome labels are to be determined. Labels obtained this way will not be pruned.

format (\%fmt) specifies the display format for the outcome labels. Default is format $(\% 6.0 \mathrm{~g})$. See $[\mathrm{D}]$ format for available formats.

suboptions are as described in $[\mathrm{G}]$ axis_label_options.

Option [y]olabel() may be repeated. Use suboptions add and custom to generate multiple sets of labels with different rendering; see [G] axis_label_options.

[y] otick (spec) adds outcome ticks on a secondary axis. otick() adds outcome ticks for the reference distribution; yotick() adds outcome ticks for the comparison distribution (only allowed after reldist cdf). The syntax of spec is

$$
\text { numlist [, suboptions] }
$$

where numlist specifies the outcome values for which ticks be generated and suboptions are as described in $[\mathrm{G}]$ axis_label_options. Option [y]otick() may be repeated. Use suboptions add and custom to generate multiple sets of ticks with different rendering; see $[\mathrm{G}]$ axis_label_options.

$[\mathrm{y}]$ oline (spec) draws added lines at the positions of the specified outcome values on a secondary axis. oline() adds outcome lines for the reference distribution; yoline() adds outcome lines for the comparison distribution (only allowed after reldist cdf). The syntax of spec is

$$
\text { numlist [, suboptions] }
$$

where numlist specifies the outcome values for which added lines be generated and suboptions are as described in $[\mathrm{G}]$ added_line_options. Option $[\mathrm{y}]$ oline() may be repeated to draw multiple sets of lines with different rendering. 
[y] otitle (tinfo) provides a title for the outcome scale axis; see [G] title_options. otitle() is for the reference distribution; yotitle() is for the comparison distribution (only allowed after reldist cdf).

\section{- Technical note}

The positions of the outcome labels, ticks, or lines are computed from information stored by reldist in e(), either from the quantiles stored in e (ogrid) or from the values stored in e (at), depending on context. There is an undocumented command called reldist olabel that can be used to compute the positions after the relative distribution has been estimated. Use this command, for example, if you want to draw a custom graph from the stored results without applying reldist graph. The syntax is as follows:

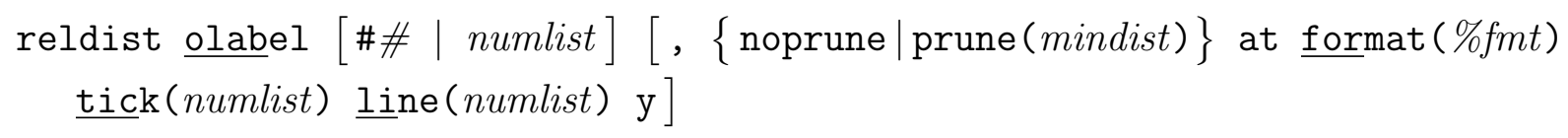

where \#\# or numlist specifies the (number of) values for which labels be generated, prune() determines the pruning (see above), at changes the meaning of the main numlist, format() specifies the display format for the labels, tick () specifies values for which ticks be generated, line() specifies values for which added lines be generated, and y request outcome labels for the $Y$ axis of the relative CDF (only allowed after reldist cdf). The command returns the following macros in $r()$ :

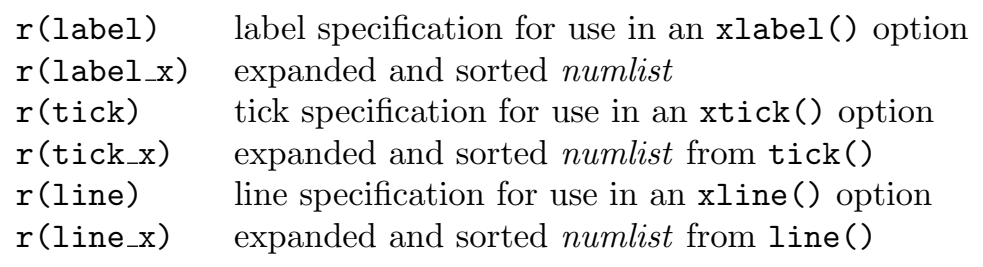

\section{General graph options}

addplot (plot) provides a way to add other plots to the generated graph. See help [G] addplot_option.

twoway_options are any options other than by() documented in help $[\mathrm{G}]$ twoway_options.

\subsection{Saved results}

reldist stores its results in e(), similar to official Stata's estimation commands. See the online documentation of reldist for details. 


\section{$5 \quad$ Examples}

\subsection{Wage mobility in two eras}

I illustrate some of the features of reldist by replicating an analysis of permanent wage growth from Handcock and Morris (1999, chapter 8). The data covers wages of white males from two cohorts of the National Longitudinal Survey, an "original" cohort started in 1966 and a "recent" cohort started in 1979. The variable of interest is the estimated growth in permanent wages between age 16 and age 34 (see Appendix C in Handcock and Morris, 1999). The data further contains information on the achieved educational level and there is a variable providing sampling weights. ${ }^{11}$

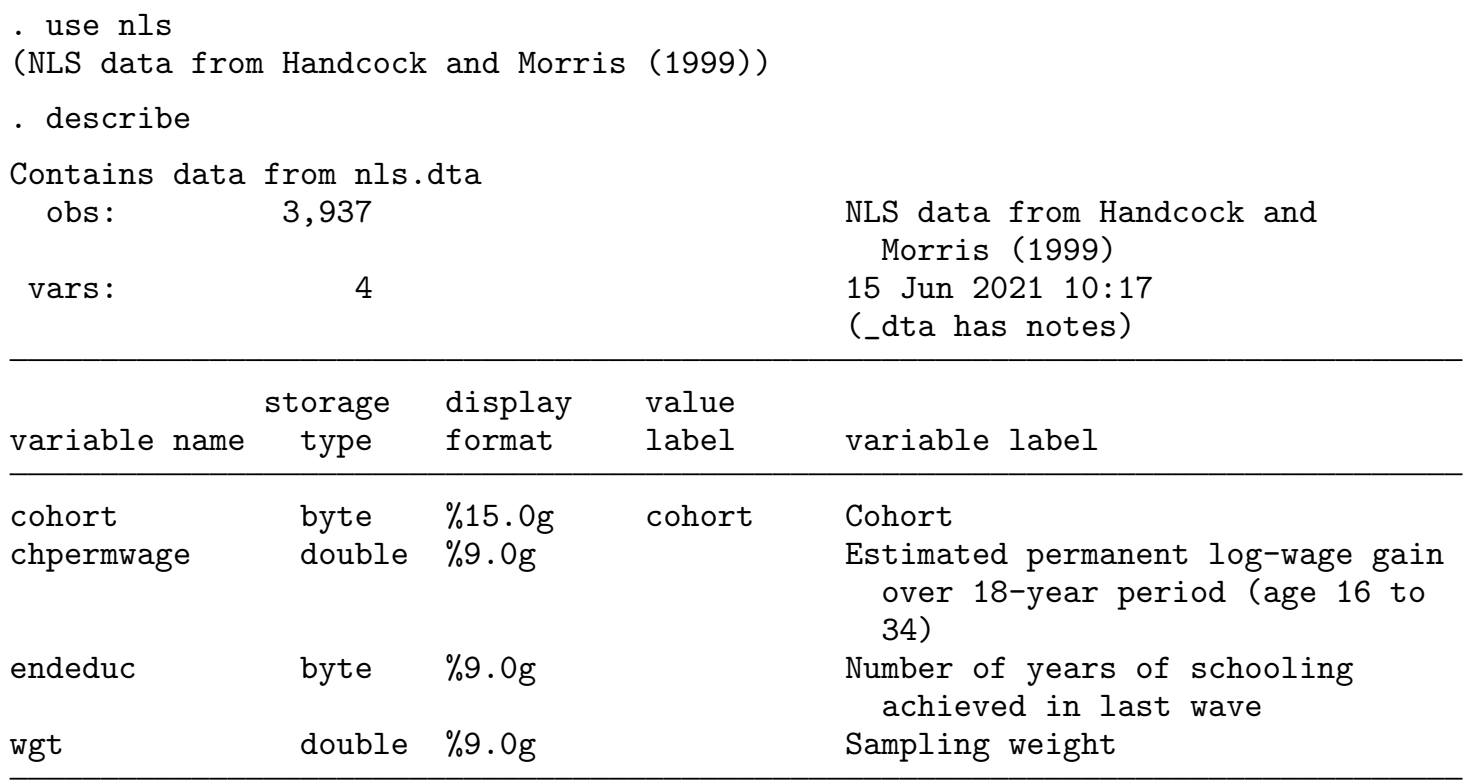

Sorted by:

- tabstat chpermwage [aw=wgt], by(cohort) stat (count mean sd med iqr) nototal

Summary for variables: chpermwage

by categories of: cohort (Cohort)

\begin{tabular}{r|rrrrr} 
cohort & $\mathrm{N}$ & mean & $\mathrm{sd}$ & $\mathrm{p} 50$ & iqr \\
\hline original (1966) & 1834 & 1.085075 & .4831473 & 1.063587 & .5812791 \\
recent (1979) & 2103 & .8782476 & .6182544 & .8535296 & .8001999 \\
\hline
\end{tabular}

Wage growth has been somewhat larger in the original cohort than in the recent cohort. The outcome variable is defined as the difference in (constant dollar) log hourly wages, so a value of 1.085 for the original cohort corresponds to a real wage growth of $\exp (1.085)-1) \times 100=$ 196 percent. For the recent cohort the average is only 0.878 (141 percent). We can also

\footnotetext{
${ }^{11}$ The data has been obtained from http://www.stat.ucla.edu/ handcock/RelDist/Data/R/RDBnls . RData.
} 
see that inequality in wage growth has been more pronounced in the recent cohort than in the original cohort, as the standard deviation of log wage gains is larger. Looking at the median and interquartile range (IQR) instead of the mean and standard deviation leads to qualitatively similar findings.

\section{The relative $\mathrm{CDF}$}

The relative CDF of log wage gains between the recent cohort and the original cohort can be obtained as follows:
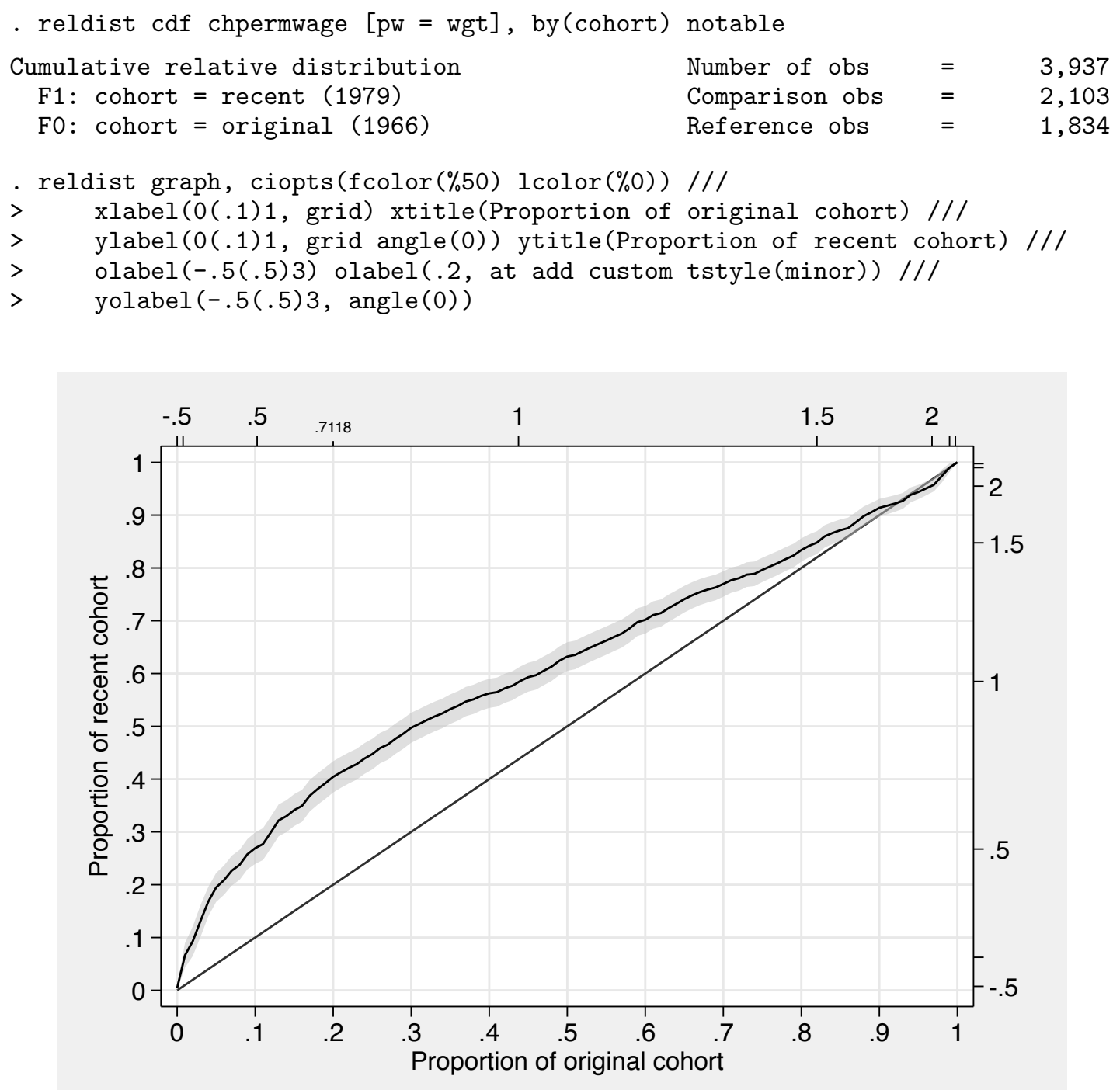

The horizontal axis of the graph corresponds to cumulative proportions of the original cohort, the vertical axis to cumulative proportions of the recent cohort, both ordered by the size of wage growth. Each point on the curve maps quantiles of the two distributions. For 
example, the value of the $20 \%$ quantile in the original cohort is equal to the $40 \%$ quantile in the recent cohort since the curve crosses point $(0.2,0.4)$. The $20 \%$ quantile in the original cohort corresponds to a log wage growth of 0.7118 , that is, a wage growth of about 104 percent. In the original cohort, 20\% experienced a wage growth of at most 104 percent; in the recent cohort, this proportion increased to $40 \%$. That is, relative to the original cohort, wage growth of 104 percent or less is overrepresented by factor 2 in the recent cohort.

\section{$\square$ Technical note}

Option notable has been applied to reldist cdf to suppress the output table containing the CDF estimate. By default, the CDF is evaluated at 101 points, so that the table would fill a whole page. Here is an example of how the table looks if we use a reduced set of evaluation points; option at(.1(.1).9) requests 9 evaluation points located at original cohort cumulative proportions $0.1,0.2, \ldots, 0.9$ :

\begin{tabular}{|c|c|c|c|c|}
\hline \multicolumn{3}{|c|}{$\begin{array}{l}\text { Cumulative relative distribution } \\
\text { F1: cohort }=\text { recent }(1979) \\
\text { F0: cohort }=\text { original }(1966)\end{array}$} & \multicolumn{2}{|c|}{$\begin{array}{l}\text { Number of obs } \\
\text { Comparison obs } \\
\text { Reference obs }\end{array}$} \\
\hline chpermwage & Coef. & Std. Err. & {$[95 \%$ Conf } & Interval] \\
\hline $\mathrm{p} 1$ & .2692422 & .0152101 & .2394219 & .2990626 \\
\hline p2 & .40432 & .01508 & .3747547 & .4338853 \\
\hline p3 & .4973859 & .0144863 & .4689846 & .5257871 \\
\hline p4 & .5624279 & .0140866 & .5348102 & .5900456 \\
\hline p5 & .6321856 & .0138188 & .605093 & .6592782 \\
\hline p6 & .7017939 & .0133607 & .6755994 & .7279883 \\
\hline p7 & .769657 & .0122928 & .7455562 & .7937579 \\
\hline p8 & .8339943 & .0112497 & .8119385 & .8560501 \\
\hline p9 & .9139871 & .0086089 & .8971088 & .9308653 \\
\hline
\end{tabular}

(evaluation grid stored in e(at))

Coefficient p2 corresponds to cumulative proportion 0.2 ; as already discussed, the value of the relative CDF is about 0.4 at this point.

Furthermore, the graph has been produced by first estimating the CDF using reldist cdf and then plotting the result using reldist graph. We could also have drawn the graph in a single step by including option graph () in the call to reldist cdf (see examples further down). Options olabel() and yolabel() have been applied to reldist graph so that additional labels are included in the graph indicating the approximate positions of specific outcome values. Labels are only printed if they are not too close together; the suppressed labels are indicated by additional ticks (this can be changed; see the description of the olabel() option above). By default, the values provided in olabel() and yolabel() are interpreted as outcome values to be included in the graph. However, if suboption at is specified, the provided values are interpreted as cumulative proportions; in this case, reldist graph will include labels for the corresponding quantiles in the graph. A second olabel() 
option has been used in this way in the command above to print the outcome value of the $20 \%$ quantile of the original cohort. ${ }^{12}$ Finally, option ciopts () has been added to make the confidence area transparent. The options specified within ciopts () are standard options for area plots; see $[\mathrm{G}]$ area_options.

\section{The relative $\mathrm{PDF}$}

Relative over- and underrepresentation of the recent cohort with respect to the distribution of wage growth in the original cohort can be seen more directly in the relative PDF. The relative PDF can be obtained as follows:

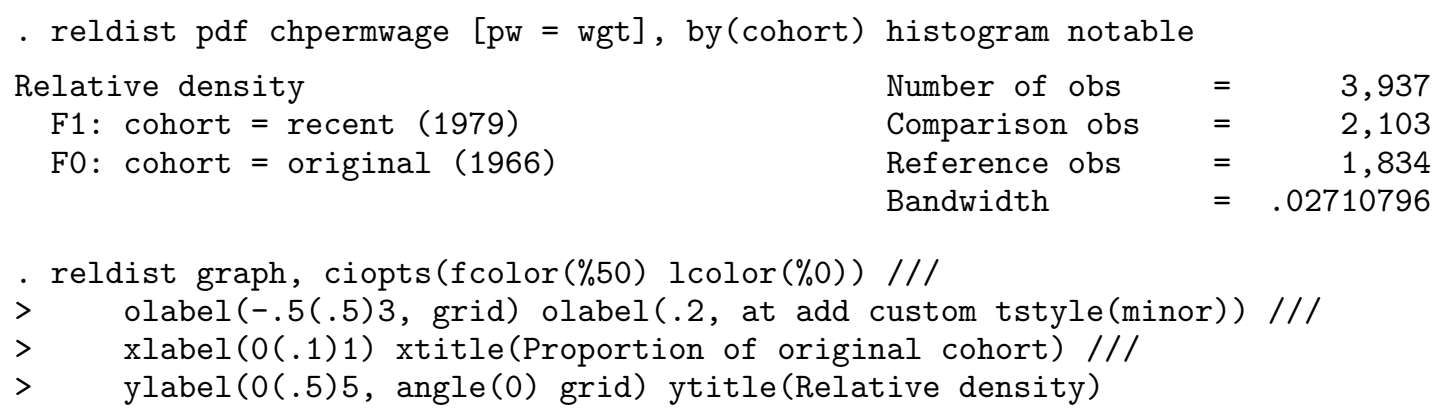

\footnotetext{
${ }^{12}$ Suboption add has been specified in the second olabel() option so that the labels from both olabel() options are printed, custom has been specified to apply custom styling to the second set of labels, and suboption tstyle(minor) selects the style. These are standard axis labelling suboptions; see [G] axis_label_options.
} 


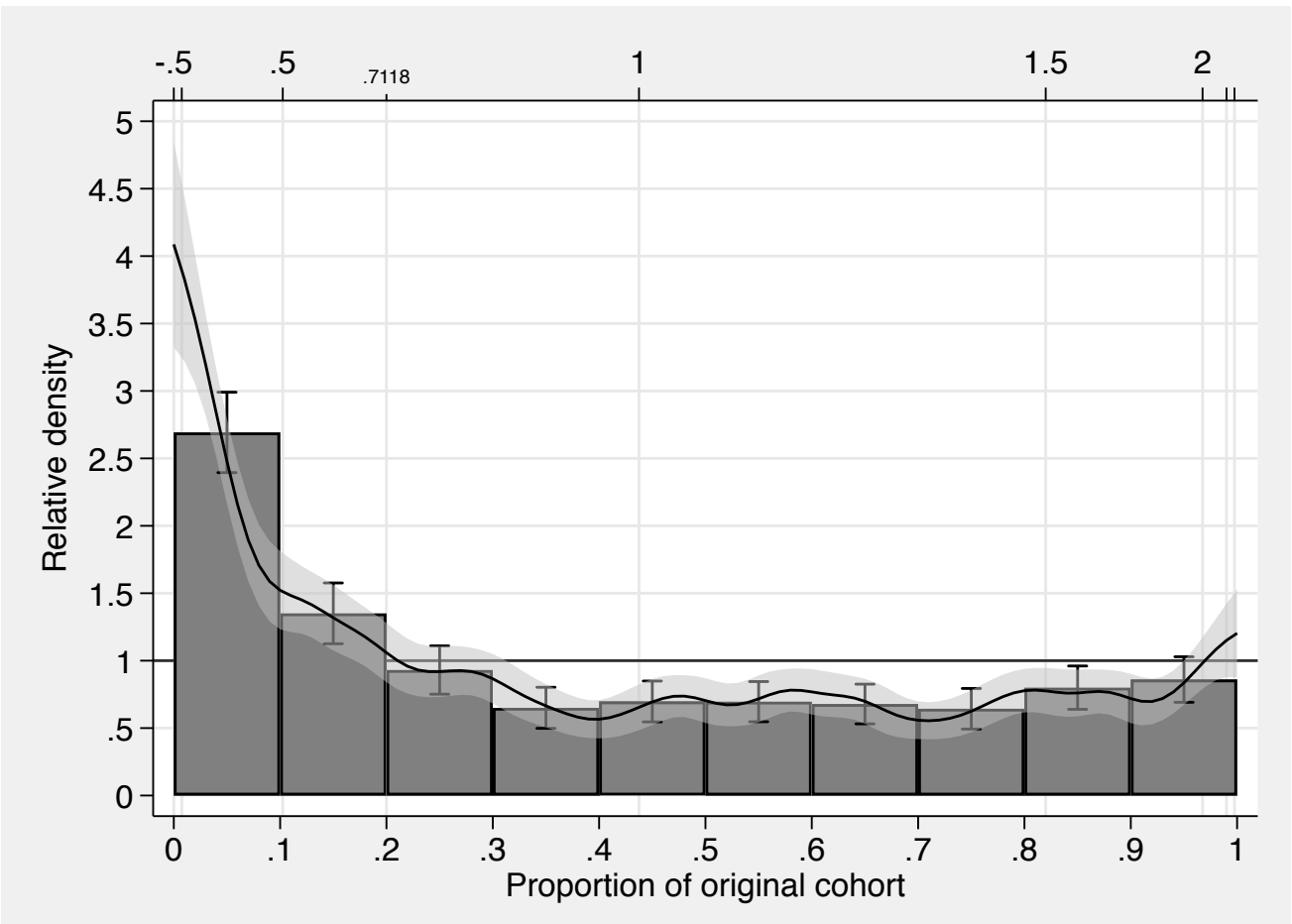

A relative density larger than one means that the recent cohort is overrepresented at the corresponding level of wage gains, values lower than one mean that the recent cohort is underrepresented relative to the original cohort. We can now directly see that the largest distributional differences are at the bottom of the distribution. The recent cohort has a much larger density than the original cohort in regions below the $10 \%$ quantile of the original cohort (overrepresentation factor of 1.5 to 4 ) and generally a larger density below about the $20 \%$ quantile. At quantiles above that, the recent cohort is underrepresented, although there is some evidence for a reduced discrepancy at the top of the distribution (above the $80 \%$ quantile) or even a reversal at the very top (above, say, the $97 \%$ quantile; although the confidence interval includes the parity line in this region, which means that the relative density is not significantly different from 1).

\section{Location and shape decomposition}

The difference in the distribution of wage gains between the original cohort and the recent cohort may have various reasons. As indicated above, wage gains have been larger on average in the original cohort than in the recent cohort, which may be due to a general difference in economic growth between the two eras that affected all population members in a similar way. In such a case, the distribution of wage gains in the recent cohort would differ from the distribution in the original cohort only in its location. However, also the structure of wage gains might have changed, for example due to rising returns on education, leading to more polarization of wage gains in the recent cohort. In this case, also the shape of the 
two distributions would be different. To separate location effects from effects of distributional differences net of location, so-called location-and-shape decompositions can be useful. reldist does not perform such decompositions directly, but it offers an option to obtain the relative distribution based on data that has been location or shape adjusted.

The following commands produce a graph containing three panels. ${ }^{13}$ The first panel shows the overall (unadjusted) relative density (same as above). The second panel shows how the relative density looks like if we only allow a difference in location but keep the distributional shape fixed. This is achieved by applying option adjust(:shape scale). The option instructs reldist to adjust the original cohort distribution in a way such that it has the same shape and scale as the recent cohort distribution, but keeps its location (technically, this is implemented by applying a location shift to the recent cohort distribution and then replacing the original cohort distribution by this counterfactual distribution; specifying scale is necessary because, conceptually, reldist treats the scale as a separate element of a distribution that can be adjusted). The third panel shows the relative density if the location difference between the two distributions is removed but the distributional shapes are allowed to be different. The corresponding option is adjust(location), which shifts the recent cohort distribution such that it has the same location as the original cohort distribution, but keeps its shape and scale. ${ }^{14}$

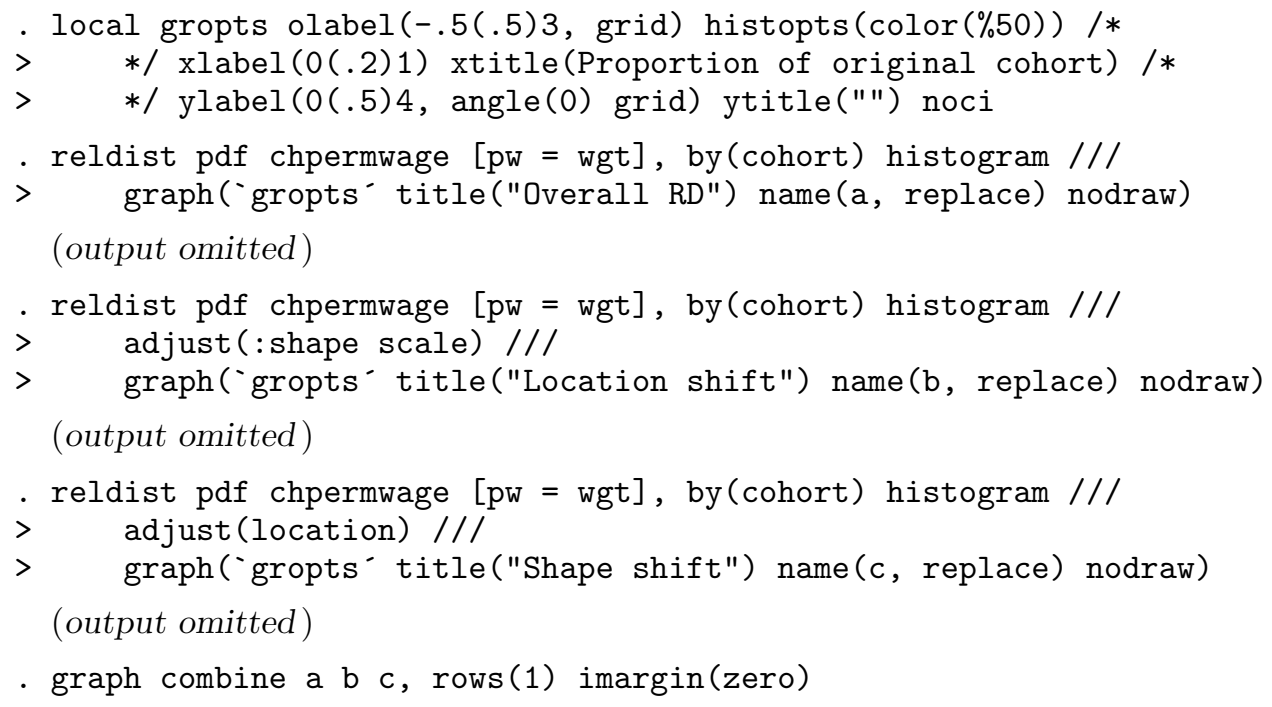

\footnotetext{
${ }^{13}$ Confidence intervals for the relative density curve have been omitted using graph option noci, so that the plots are less busy.

${ }^{14}$ Handcock and Morris (1999) do the decomposition the other way round, equivalent to specifying adjust(shape scale) and adjust(:location).
} 


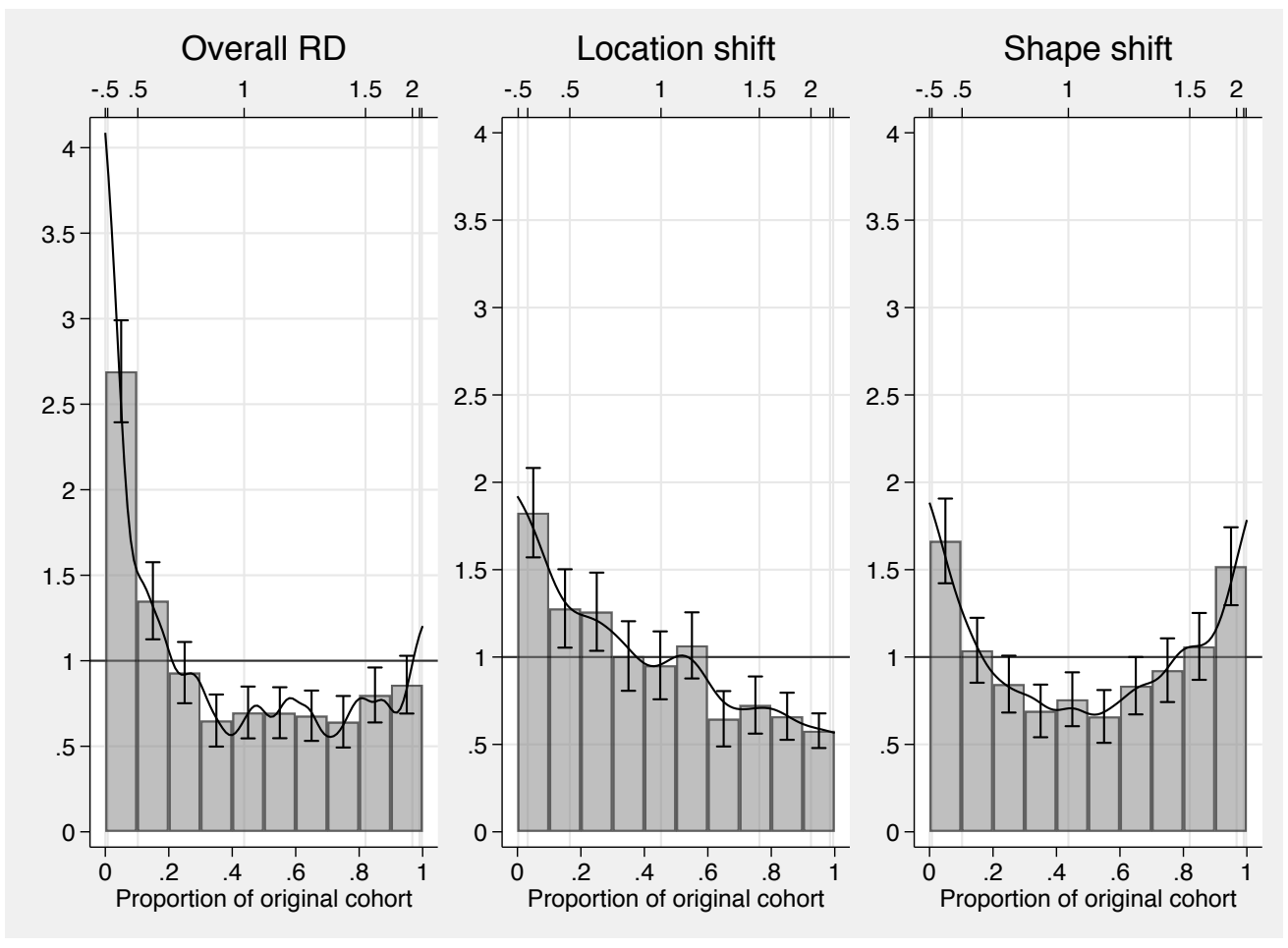

The results indicate that the difference between the recent cohort distribution and the original cohort distribution is not only a matter of location, but that there is also a substantial difference in distributional shape. In particular, the recent cohort distribution appears more polarized than the original cohort (also see below).

\section{Distributional divergence}

To determine the relative contributions of location and shape differences to the overall distributional divergence between the two cohorts, Handcock and Morris (1999) suggest comparing the entropy (Kullback-Leibler divergence) of the unadjusted and adjusted relative distributions. Such an analysis can be obtained by reldist divergence: ${ }^{15}$

\begin{tabular}{|c|c|c|c|}
\hline Relative distribution divergence & Number of obs & $=$ & 3,937 \\
\hline F1: cohort $=$ recent $(1979)$ & Comparison obs & $=$ & 2,103 \\
\hline F0: cohort $=$ original $(1966)$ & Reference obs & $=$ & 1,834 \\
\hline Adjustment (alternate model) & Histogram bins & $=$ & 20 \\
\hline $\begin{array}{l}\text { F1: location } \\
\text { F0: (none) }\end{array}$ & Statsistic & $=$ & entropy \\
\hline
\end{tabular}

\footnotetext{
${ }^{15}$ Alternative measures offered by reldist divergence are the Chi-squared divergence and the dissimilarity index (total variation distance).
} 


\begin{tabular}{r|rccr} 
chpermwage & Coef. & Std. Err. & \multicolumn{2}{c}{ [95\% Conf. Interval] } \\
\hline main & .1726182 & .021244 & .1309679 & .2142686 \\
alternate & .0670518 & .0126801 & .0421917 & .091912 \\
difference & .1055664 & .0179497 & .0703748 & .140758 \\
\hline
\end{tabular}

Three divergence values are reported in the above output: the divergence of the unadjusted relative distribution (labelled as main), the divergence of the relative distribution after location-adjusting the recent cohort (labelled as alternate), as well as the difference between these two measures. The first value is the overall divergence, the second value quantifies the divergence due to differences in distributional shape, and the third value quantifies the contribution of the difference in location. ${ }^{16}$ We can use $[\mathrm{R}]$ nlcom to compute the percentage contributions of the location and shape effects to the overall divergence:

\begin{tabular}{|c|c|c|c|c|c|c|}
\hline $\begin{array}{r}\text { loc: } \\
\text { shape: }\end{array}$ & $\begin{array}{l}\text { _b [differen } \\
\text { _b [alternat }\end{array}$ & $\begin{array}{l}\mathrm{e}] / \mathrm{b}[\mathrm{main} \\
\mathrm{i} / \mathrm{b}[\mathrm{main}]\end{array}$ & & & & \\
\hline chpermwage & Coef. & Std. Err. & z & $P>|z|$ & {$[95 \%$ Conf. } & Interval] \\
\hline loc & 61.15599 & 6.246685 & 9.79 & 0.000 & 48.91271 & 73.39927 \\
\hline shape & 38.84401 & 6.246685 & 6.22 & 0.000 & 26.60073 & 51.08729 \\
\hline
\end{tabular}

We see that in this example the difference in location appears to be more relevant (60\%) than the difference in shape (40\%). Qualitatively, the results are similar to the ones reported by Handcock and Morris (1999), but note that the precise values are different. On the one hand, Handcock and Morris (1999) performed a slightly different decomposition (see Footnote 16). More importantly, however, the Kullback-Leibler divergence is quite sensitive to the details of the computation of the underlying relative density. By default, reldist divergence obtains the divergence from a 20-bin histogram; changing the number of bins may change the results substantially. Furthermore, the divergence measures could also be obtained from a kernel density estimate of the relative density (see option pdf), which would yield yet another set of results (substantially depending on the bandwidth).

\section{Polarization analysis}

As stated above, the recent cohort distribution appears more polarized than the original cohort distribution. A measure to quantify the polarization is the MRP computed by reldist mrp:

\footnotetext{
${ }^{16}$ As discussed above, the last value has a cross-entropy interpretation. Note that reldist divergence could also be used to compute alternative decompositions, for example, between the overall relative distribution and a shape-adjusted relative distribution, treating the location effect as a cross-entropy (as in Handcock and Morris, 1999).
} 


\begin{tabular}{|c|c|c|c|c|c|c|c|}
\hline \multicolumn{3}{|c|}{$\begin{array}{l}\text { Median relative polarization } \\
\text { F1: cohort = recent (1979) } \\
\text { F0: cohort = original (1966) } \\
\text { Adjustment: location }\end{array}$} & & \multicolumn{2}{|c|}{$\begin{array}{l}\text { Number of obs } \\
\text { Comparison obs } \\
\text { Reference obs }\end{array}$} & $\begin{array}{l}= \\
= \\
=\end{array}$ & $\begin{array}{l}3,937 \\
2,103 \\
1,834\end{array}$ \\
\hline chpermwage & Coef. & Std. Err. & t & $P>|t|$ & {$[95 \%$} & Conf. & Interval] \\
\hline MRP & .1832597 & .0191808 & 9.55 & 0.000 & .1456 & 5544 & .220865 \\
\hline LRP & .190353 & .0303527 & 6.27 & 0.000 & .1308 & 3445 & .2498615 \\
\hline URP & .1761664 & .0291428 & 6.04 & 0.000 & & 1903 & .2333029 \\
\hline
\end{tabular}

The results indicate that the recent cohort distribution is indeed more polarized, as the value of the MRP is positive, of substantial magnitude (the possible range of the MRP is between -1 and 1), and significantly different from zero. Furthermore, the breakup into polarization of the lower half (LRP) and the upper half of the distribution (URP) suggests that the degree of relative polarization is similar in both tails.

\section{Covariate balancing}

Education may be on important determinant of the wage distribution as well as the distribution of wage gains over an occupational career. Hence, if the educational distribution changed between the original cohort and the recent cohort, we may be comparing apples with oranges. That is, one reason for the difference in the distribution of wage gains in the two cohorts may be that the cohorts have a different educational composition. This indeed seems to be the case, if we look at the relative density of educational levels between the cohorts: ${ }^{17}$

\begin{tabular}{|c|c|c|c|c|c|}
\hline \multicolumn{6}{|c|}{$\begin{array}{l}\text {. replace endeduc }=8 \text { if endeduc }<8 \\
\text { (34 real changes made) } \\
\begin{array}{ll}\text { - reldist pdf endeduc }[\mathrm{pw}=\text { wgt], by (cohort) categorical } \\
\text { Relative density } & \text { Number of obs } \\
\text { F1: cohort }=\text { recent (1979) } & \text { Comparison obs } \\
\text { F0: cohort = original (1966) } & \text { Reference obs }\end{array}\end{array}$} \\
\hline endeduc & Coef. & Std. Err. & {$[95 \%$} & Conf & Interval] \\
\hline \multicolumn{6}{|l|}{ endeduc } \\
\hline 8 & .9383436 & .220892 & & 0527 & 1.371417 \\
\hline 9 & 1.485883 & .3551772 & .7895 & 5346 & 2.182232 \\
\hline 10 & 1.59819 & .3734487 & .8660 & 0189 & 2.330361 \\
\hline 11 & .9276922 & .1673159 & .5996 & 6581 & 1.255726 \\
\hline 12 & 1.41295 & .0657943 & & 3956 & 1.541945 \\
\hline
\end{tabular}

\footnotetext{
${ }^{17}$ Option categorical instructs reldist to treat endeduc as a factor variable and to compute the relative density as the ratio of relative frequencies between the two cohorts at each level. Confidence intervals have been suppressed in the graph using option noci.
} 


\begin{tabular}{r|rrrr}
13 & 1.012919 & .1136963 & .7900094 & 1.235828 \\
14 & .7208455 & .0737943 & .5761668 & .8655241 \\
15 & .6660931 & .0984461 & .473083 & .8591032 \\
16 & .8683801 & .0644533 & .7420152 & .994745 \\
17 & .5069374 & .0751882 & .3595259 & .6543489 \\
18 & .7644302 & .0823954 & .6028885 & .9259719 \\
\hline \\
(evaluation grid stored in e(at)) \\
. reldist graph, noci olabel(8(1)18, prune(.05)) /// \\
$>\quad$ xlabel(0(.1)1) xtitle(Proportion of original cohort) /// \\
$>\quad$ ylabel(0(.2)1.6, angle(0) grid) ytitle(Relative density)
\end{tabular}

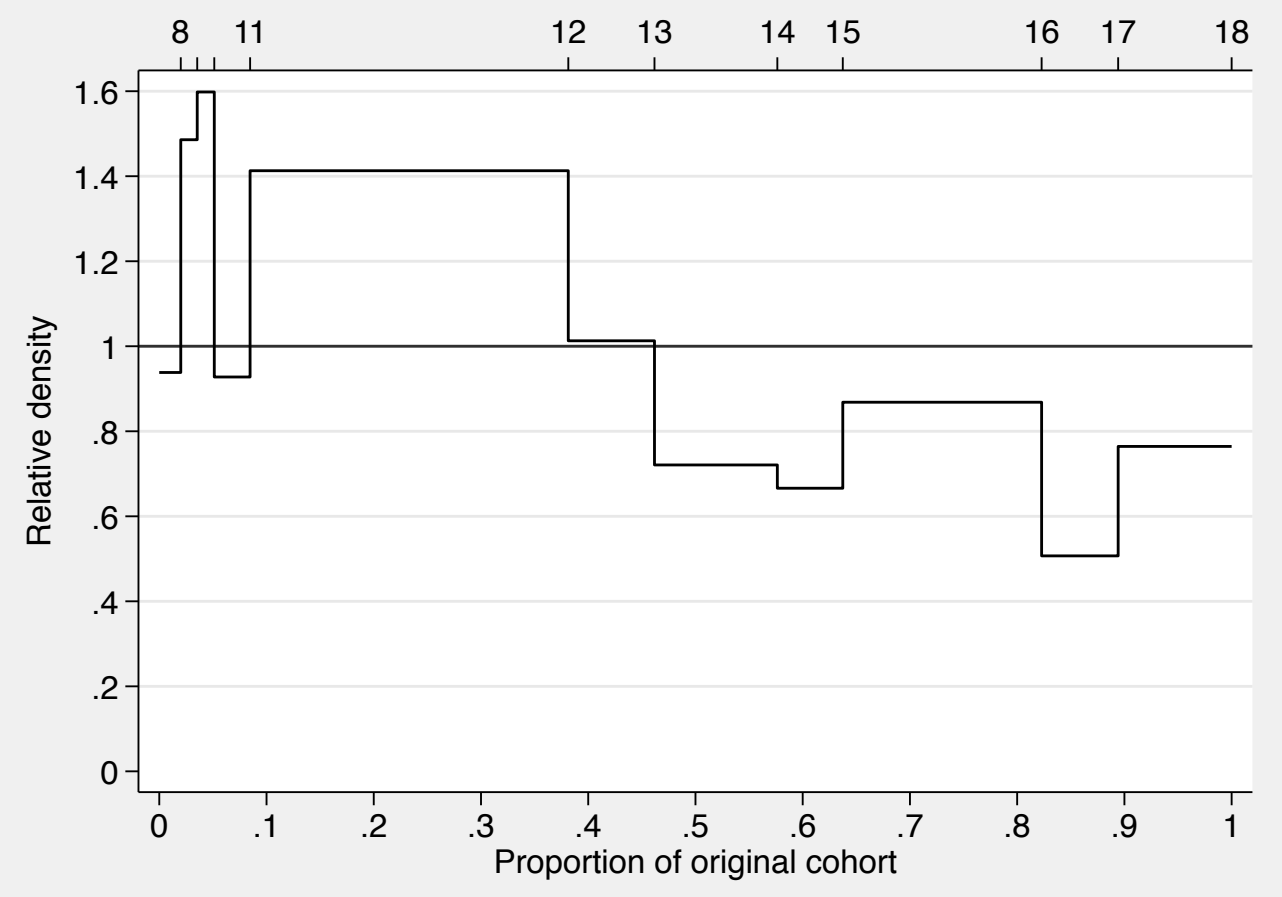

Lower educational levels appear to be more frequent in the recent cohort than in the original cohort (relative density mostly larger than one), higher educational levels appear to be less frequent (relative density below one). Looking at the table we see that in many cases the confidence interval does not include one, meaning that these differences between the cohorts are statistically significant.

As suggested by Handcock and Morris (1999), the graph above uses a step function with the steps located at the values of the cumulative distribution in the original cohort. An alternative would be to display the categorical relative density as a histogram in which the width of each bar is proportional to the relative frequency of the corresponding level in the original cohort:

- reldist hist endeduc $[\mathrm{pw}=\mathrm{wgt}]$, by (cohort) categorical /// 

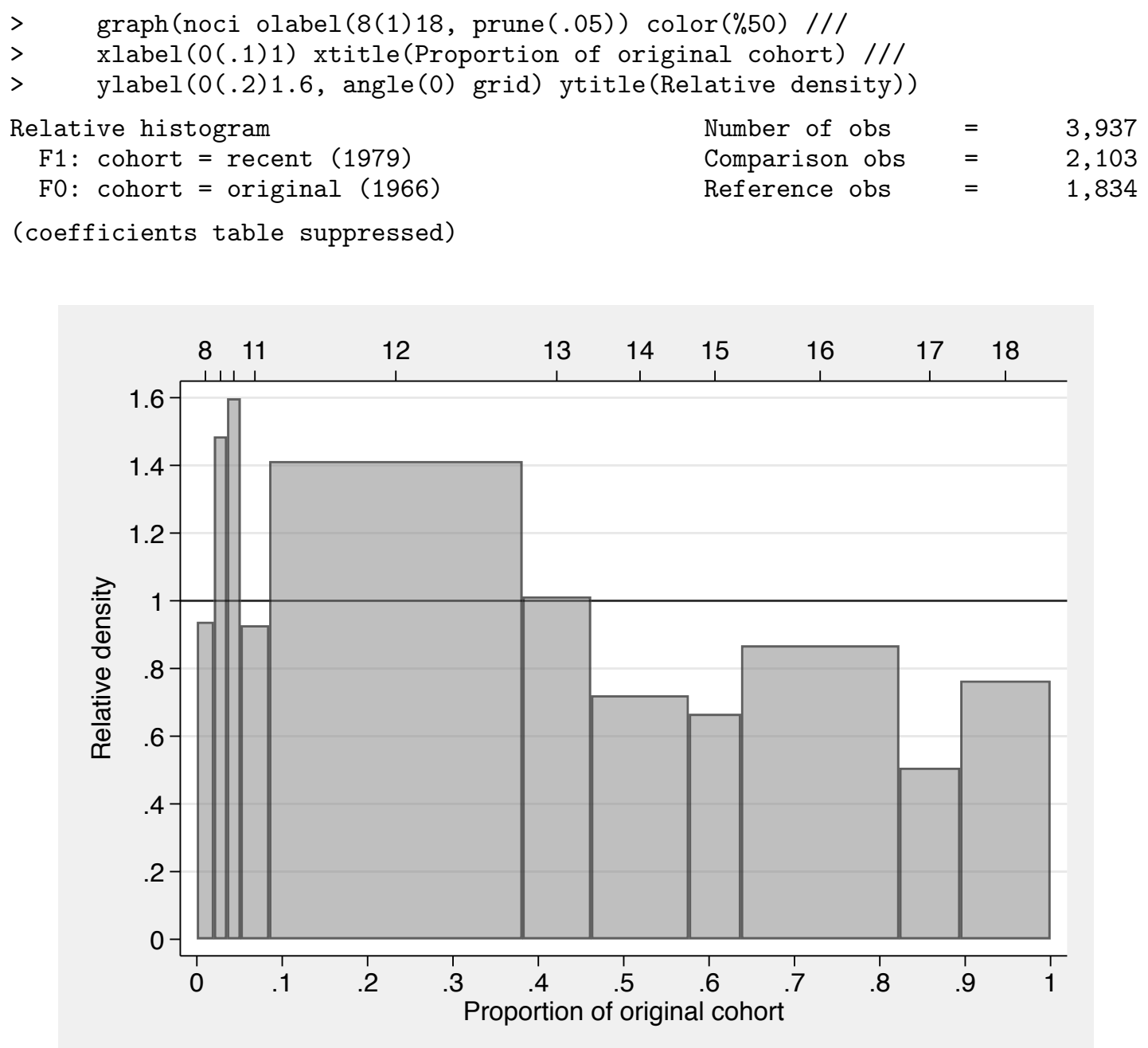

Numerically, both approaches lead to the exact same results (including standard errors and confidence intervals), but a different style is used for graphical display.

The question now is whether these differences in educational composition affect the relative distribution of wage gains. Similar as above in the context of location and shape effects, we can identify the contribution of compositional differences by comparing unadjusted and adjusted relative distributions. The adjustment, however, is now accomplished by reweighting one of the distributions in a way such that its educational composition becomes equal to the educational composition in the other cohort. Option balance() can be used in reldist to apply such balancing. Here is an example that displays the overall relative distribution (left panel), the relative distribution after the recent cohort has been reweighted (right panel), as well as the relative distribution between the raw and reweighted recent cohort (middle panel; the purpose of the middle panel is to show how reweighting changes the distribution of the recent cohort):

- local gropts olabel(-.5(.5)3, grid) histopts $(\operatorname{color}(\% 50)) / *$ 

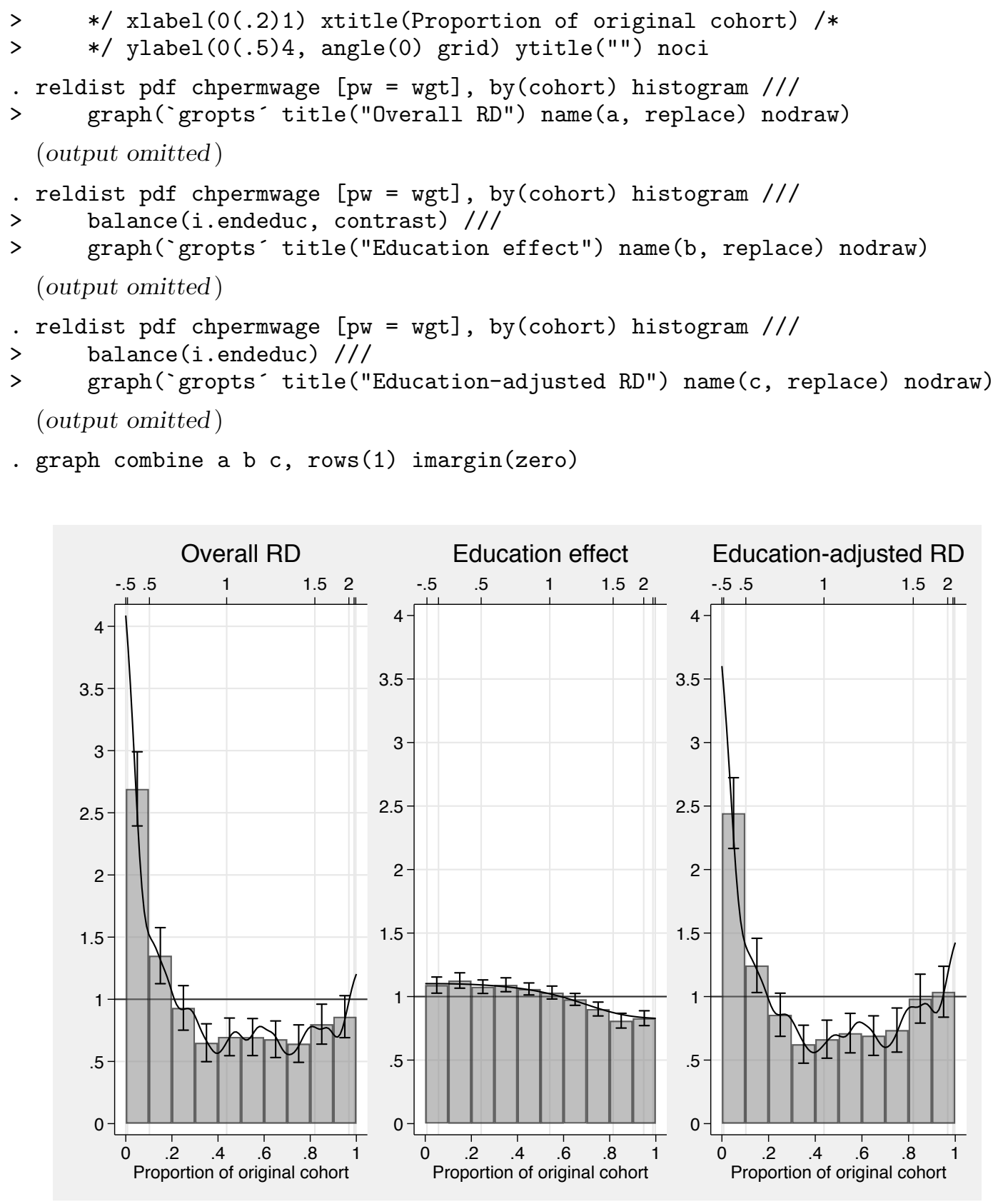

Adjusting the educational composition does seem to make the distribution of wage gains somewhat more equal between the two cohorts. The comparison between the raw recent cohort and the reweighted recent cohort (middle panel) shows that low (high) wage gains are more (less) frequent in the raw data than in the reweighted data. That is, as expected, reweighting the recent cohort generally shifts the distribution of wage gains upwards, thus making it more equal to the distribution of wage gains in the original cohort (the effect of the reweighting is statistically significant, as can be inferred from the confidence intervals that have been included for the histogram). Overall, however, the contribution of the difference 
in educational composition only seems to be of minor importance: there is only a small difference between the overall relative distribution (left panel) and the education-adjusted relative distribution (right panel).

\section{Location adjustment by means of covariate balancing}

Note that reweighting can be used as an alternative method for location adjustments. The default method, provided by option adjust(), implements the adjustments by transforming the outcome values. The same goal, however, can also be reached by altering the PDF of the data while leaving the outcome values unchanged. This is what reweighting does if we include the outcome variable in the balancing equation. Here is a replication of the locationand-shape decomposition from above using balance() instead of adjust(). I use entropy balancing to obtain the weights, which ensures that the means of the two distribution will be exactly the same:

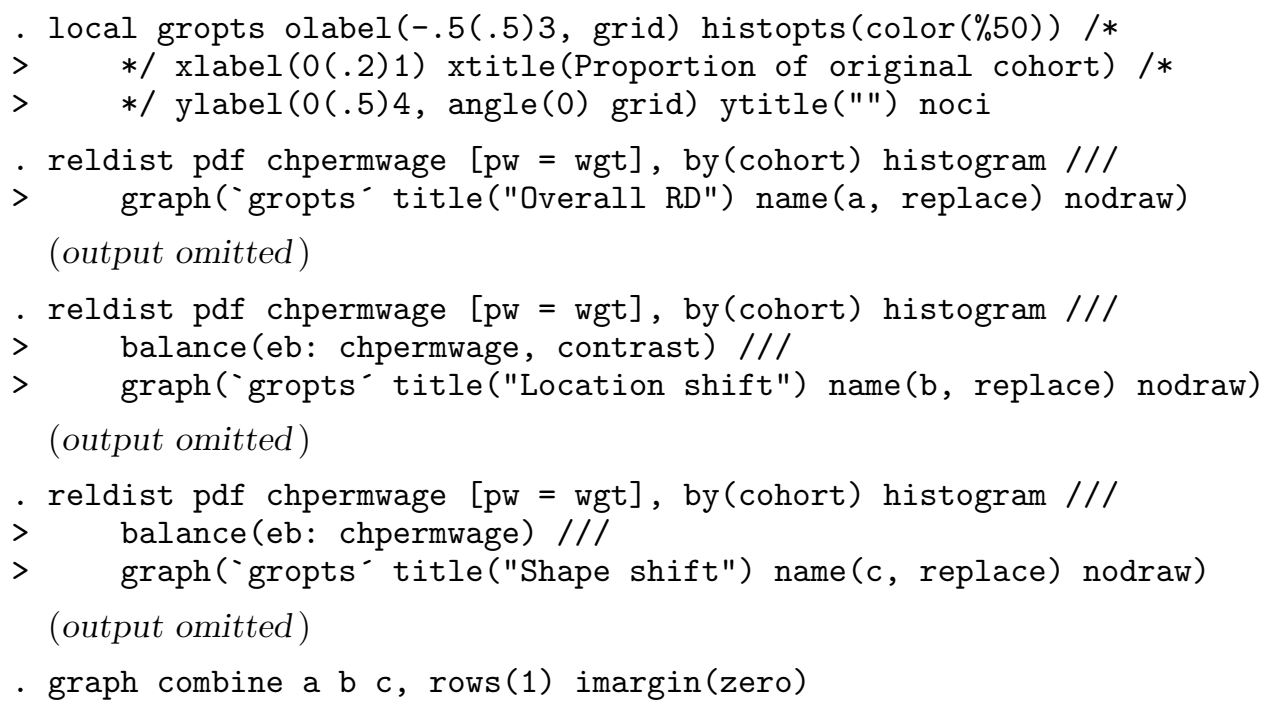




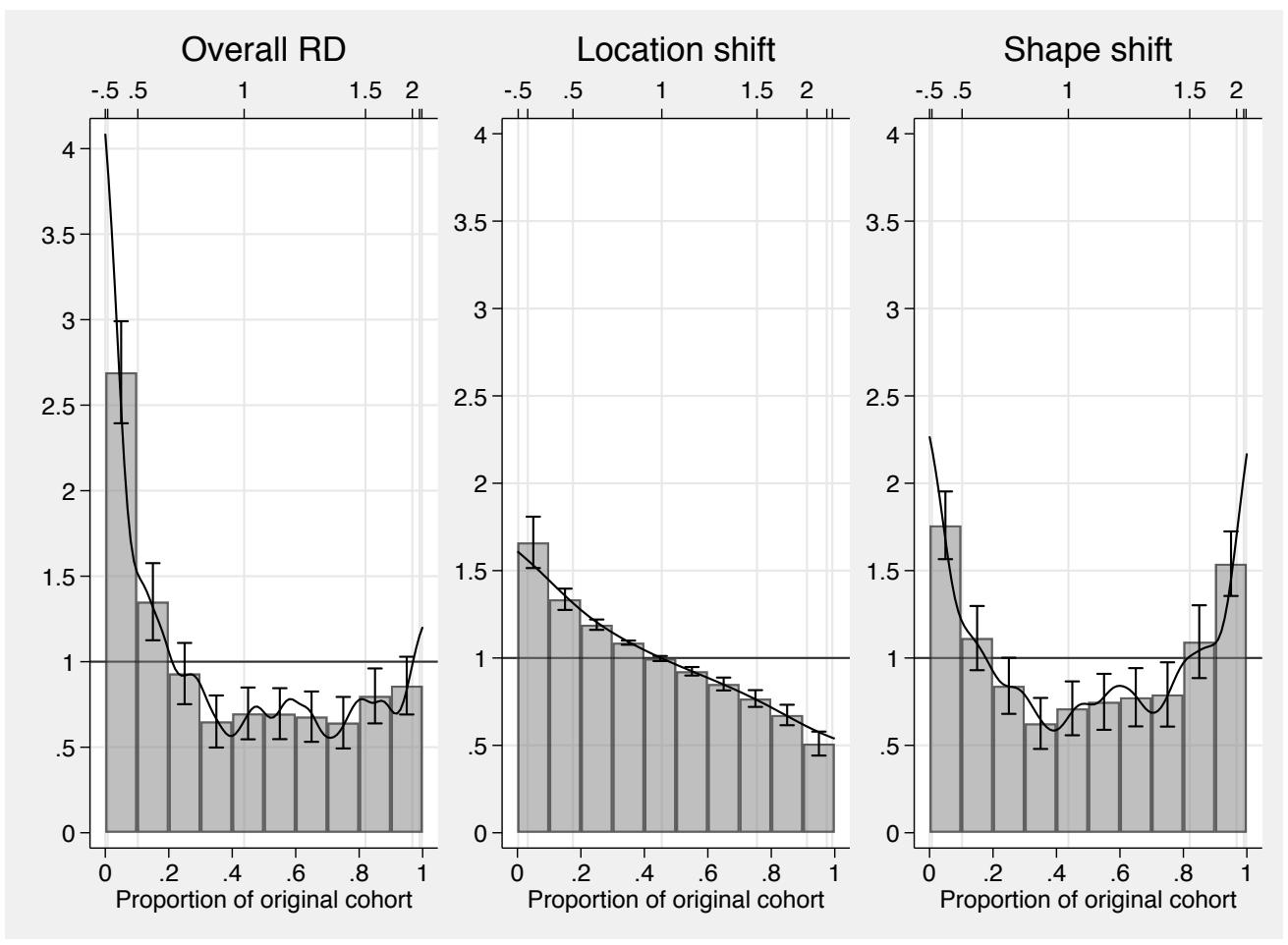

The two approaches lead to qualitatively similar results. ${ }^{18}$ One advantage of the reweighting approach, however, is that heaping in the data will have less adverse effects on the results. $^{19}$

\subsection{Processing results from reldist}

\section{Post-estimation hypothesis testing}

reldist stores its results in e() just like any other estimation command. Hence, we can use post-estimation commands such as $[\mathrm{R}]$ test to test hypotheses or coefplot (Jann, 2014) to draw graphs.

I use the NLSW 1988 data shipped with Stata to analyze wages of unionized and nonunionized workers. For example, we might be interested in relative wage polarization. An obvious hypothesis is that wages are more polarized among non-unionized workers than among the unionized, but the pattern may be different depending on education. Here are the results for the MRP between non-unionized and unionized workers for different levels of qualification:

\footnotetext{
${ }^{18}$ Although note that adjust (), as used above, adjusts the medians of the distributions whereas balance() adjusts the means. For a more valid comparison suboption mean could be specified within adjust().

${ }^{19}$ Note that reweighting could be used for location-and-scale adjustment by including the square of the outcome variable as an additional covariate in the balancing equation.
} 


\begin{tabular}{|c|c|c|c|c|c|c|c|}
\hline \multicolumn{8}{|c|}{$\begin{array}{l}\text {. sysuse nlsw88, clear } \\
\text { (NLSW, } 1988 \text { extract) } \\
\text {. reldist mrp wage, by(union) swap over(collgrad) multiplicative }\end{array}$} \\
\hline \multicolumn{4}{|c|}{$\begin{array}{l}\text { Median relative polarization } \\
\begin{aligned} & \text { F1: union }=\text { nonunion } \\
& \text { F0: union }=\text { union } \\
& \text { Adjustment: location (mult) } \\
& 0: \text { collgrad }=\text { not college grad } \\
& 1: \text { collgrad }=\text { college grad }\end{aligned}\end{array}$} & \multicolumn{3}{|c|}{$\begin{array}{ll}\text { Number of obs } & = \\
\text { Comparison obs } & = \\
\text { Reference obs } & =\end{array}$} & $\begin{array}{r}1,878 \\
1,417 \\
461\end{array}$ \\
\hline wage & Coef. & Std. Err. & $\mathrm{t}$ & $P>|t|$ & {$[95 \%$} & Conf. & Interval] \\
\hline \multicolumn{8}{|l|}{0} \\
\hline MRP & .0654444 & .0358179 & 1.83 & 0.068 & -.0048 & 3027 & .1356916 \\
\hline LRP & -.0015699 & .0572336 & -0.03 & 0.978 & -.113 & 3818 & .1106783 \\
\hline URP & .1324587 & .0571956 & 2.32 & 0.021 & .020 & 285 & .2446324 \\
\hline \multicolumn{8}{|l|}{1} \\
\hline MRP & .1486059 & .0591766 & 2.51 & 0.012 & .032 & 2547 & .2646647 \\
\hline LRP & .1985118 & .0818497 & 2.43 & 0.015 & .0379 & 9858 & .3590378 \\
\hline URP & .0987 & .0920773 & 1.07 & 0.284 & -.0818 & 3847 & .2792846 \\
\hline
\end{tabular}

Option swap has been specified to flip the two groups, so that the non-unionized are the comparison group and the unionized are the reference group. Option multiplicative has been specified because - based on economic theory - a proportional location shift makes more sense for wages than an additive shift.

As hypothesized, the results suggest that wage polarization is generally more pronounced among non-unionized workers, although the MRP is only marginally significant for respondents without college degree. A follow-up question might thus be whether we can conclude from the data that relative polarization between non-unionized and unionized workers is stronger among college graduates than among workers without college degree. We can use test to test the two MRP estimates against each other:

$$
\begin{aligned}
& \text {. test [0]MRP }=[1] \mathrm{MRP} \\
& \text { (1) }[0] \mathrm{MRP}-[1] \mathrm{MRP}=0 \\
& F(1,1877)=1.45 \\
& \text { Prob }>F=0.2294
\end{aligned}
$$

The test is negative; that is, we cannot reject the null hypothesis that the two MRP estimates are the same ( $p$-value of 0.229$)$. The same result can also be obtained using $[\mathrm{R}]$ lincom:

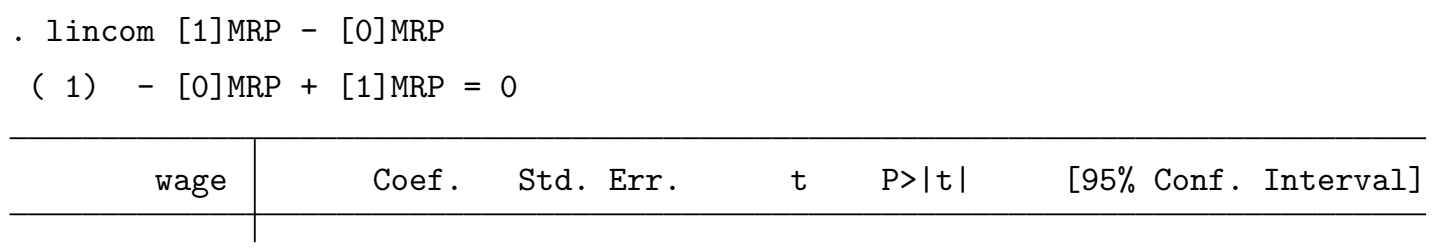




\section{Creating graphs from multiple results}

When comparing wages between unionized and non-unionized workers it may be relevant to make the two groups more comparable by taking background characteristics into account. Possibly, some of the difference in the wage distributions is due to differential composition with respect to these characteristics, and not due to unionization status per se. Here is how you could plot the relative density curves based on raw data and on balanced data in a single graph using $[\mathrm{R}]$ estimates store and coefplot (Jann, 2014):
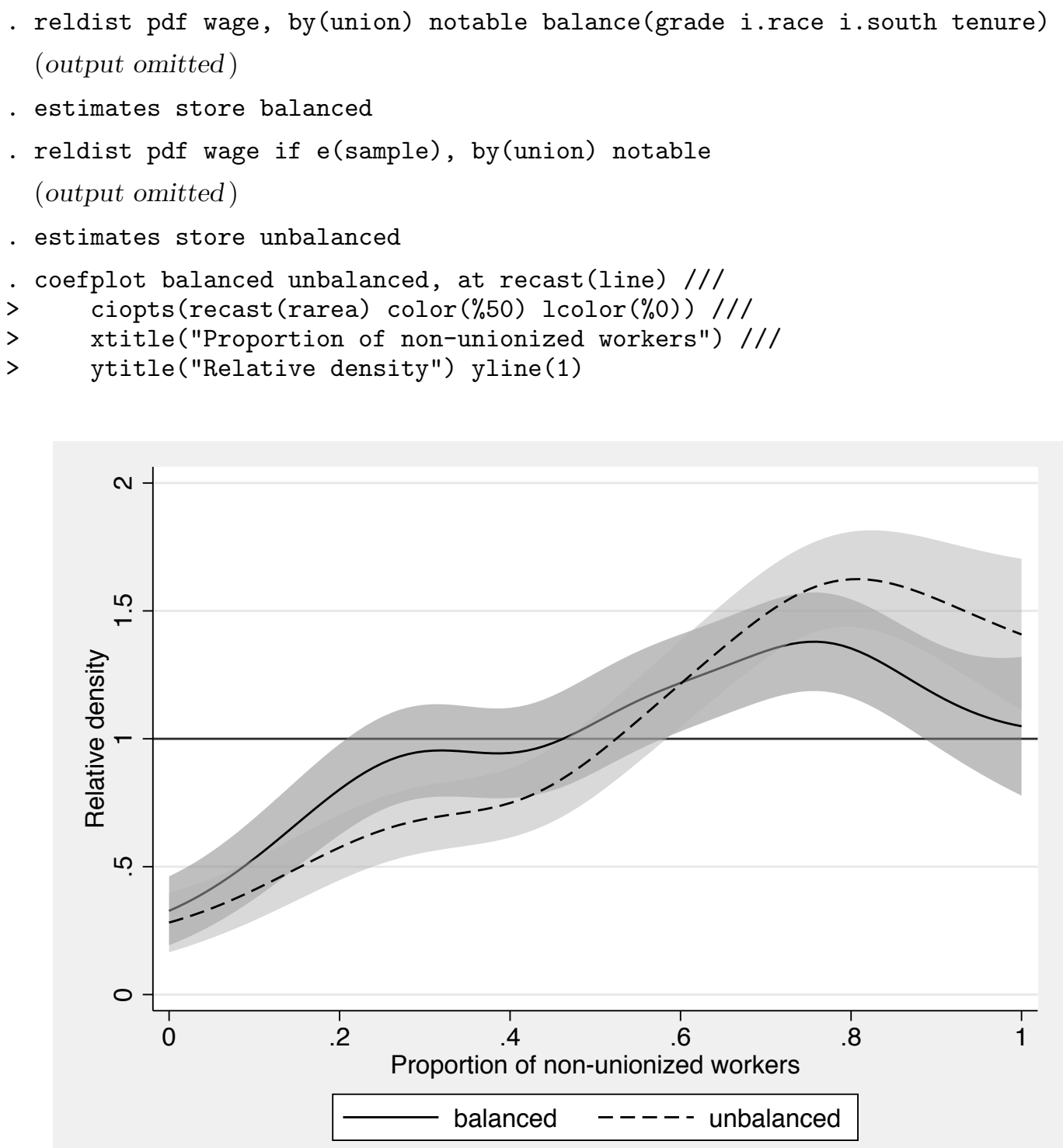
We see that the wage distributions of unionized and non-unionized workers become more similar once we control for background characteristics, especially in the upper part of the distribution.

\section{Working with influence functions}

The predict command can be used to store the influence functions that reldist uses for standard error estimation. For example, we may want to test whether relative polarization between non-unionized and unionized workers is more pronounced for wages than for working hours. reldist does not support analyzing two variables at the same time. However, we can store the influence functions and then use them to test the MRP for wages against the MRP for working hours:

\begin{tabular}{|c|c|c|c|c|c|c|c|}
\hline \multicolumn{3}{|c|}{$\begin{array}{l}\text { Median relative polarization } \\
\text { F1: union = nonunion } \\
\text { F0: union = union } \\
\text { Adjustment: location (mult) }\end{array}$} & & \multicolumn{2}{|c|}{$\begin{array}{l}\text { Number of obs } \\
\text { Comparison obs } \\
\text { Reference obs }\end{array}$} & $\begin{array}{l}= \\
= \\
=\end{array}$ & $\begin{array}{r}1,877 \\
1,416 \\
461\end{array}$ \\
\hline wage & Coef. & Std. Err. & $\mathrm{t}$ & $P>|t|$ & {$[95 \%$} & Conf & Interval] \\
\hline MRP & .123268 & .0303101 & 4.07 & 0.000 & .06 & 3823 & .182713 \\
\hline LRP & .0573649 & .0494239 & 1.16 & 0.246 & -.039 & 5667 & .1542964 \\
\hline URP & .1891712 & .0482137 & 3.92 & 0.000 & .09 & 4613 & .2837294 \\
\hline
\end{tabular}

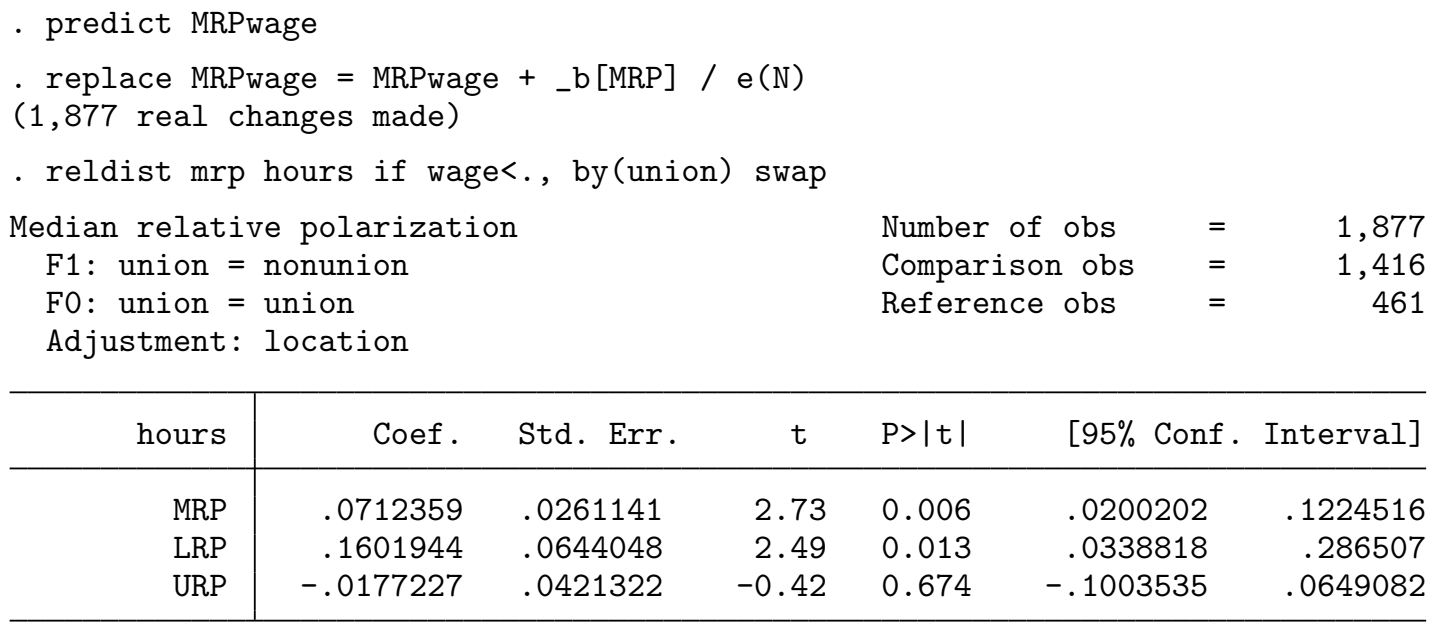

- predict MRPhours

- replace MRPhours = MRPhours + _b [MRP] / e(N)

(1,877 real changes made)

- total MRPwage MRPhours

Total estimation Number of obs $\quad$ N $\quad 1,877$ 


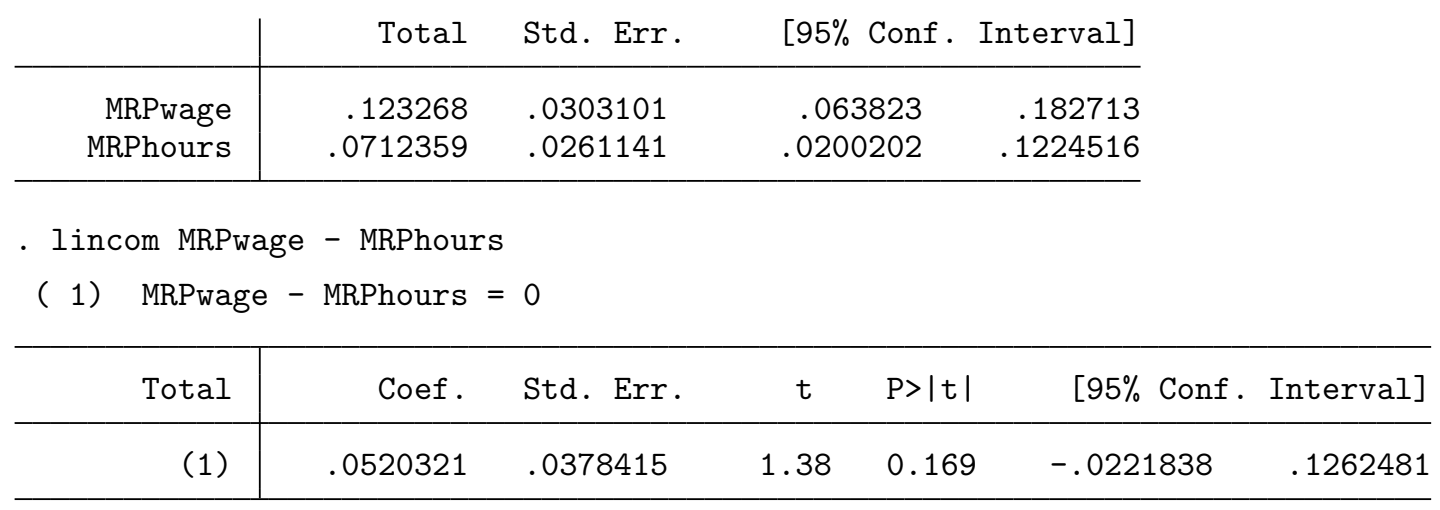

- drop MRPwage MRPhours

The MRP is higher for wages than for working hours, but the difference does not appear to be statistically significant. In the example I first stored the influence functions and then recenterd them by adding the point estimates back in (on the use of recentered influence functions see, e.g., Firpo et al., 2009, and Rios-Avila, 2020). The influence functions returned by reldist are scaled such that $[\mathrm{R}]$ total can be used for estimation of standard errors (note how $[\mathrm{R}]$ total reproduced the results from reldist in the example). This is why I divided the point estimate by $N$ before adding it back in. Alternatively, multiply the influence function by $N$, add the point estimate as is, and then use $[\mathrm{R}]$ mean instead of $[\mathrm{R}]$ total. Furthermore, note that weights are not incorporated into the influence functions. That is, if weights have been applied to reldist, the weights will also have to be applied when calling [R] total or $[\mathrm{R}]$ mean (the same is true for clustering).

\subsection{Survey estimation}

reldist fully supports estimation for complex survey data, but the [SVY] svy prefix command cannot be used for technical reasons if the variance estimation method is set to linearized (Taylor-linearized variance estimation). You can use option vce(svy) instead of the svy prefix in this case. Example:

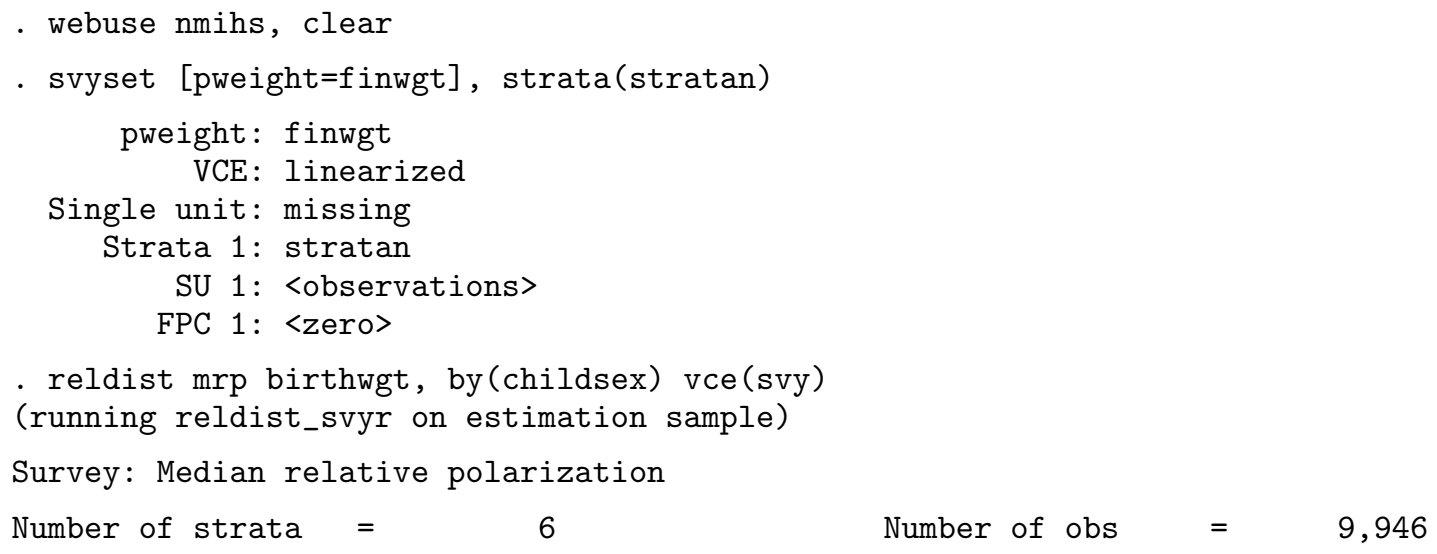




\begin{tabular}{|c|c|c|c|c|c|c|c|}
\hline Number of PSU & \multicolumn{2}{|c|}{9,946} & & \multicolumn{2}{|c|}{ Population size } & $\begin{array}{l}= \\
=\end{array}$ & $\begin{array}{r}3,895,562 \\
9,940\end{array}$ \\
\hline F1: childse & $=2$ & & & \multicolumn{2}{|c|}{ Comparison obs } & $=$ & 4,911 \\
\hline F0: childse & $=1$ & & & \multicolumn{2}{|c|}{ Reference obs } & $=$ & 5,035 \\
\hline & & Linearized & & & & & \\
\hline birthwgt & Coef. & Std. Err. & t & $P>|t|$ & {$[95 \%$} & Conf. & Interval] \\
\hline MRP & -.0349405 & .0155133 & -2.25 & 0.024 & -.0653 & 496 & -.0045313 \\
\hline LRP & .0024726 & .0233231 & 0.11 & 0.916 & -.0432 & 454 & .0481907 \\
\hline URP & -.0723535 & .0252147 & -2.87 & 0.004 & -.1217 & 795 & -.0229275 \\
\hline
\end{tabular}

Results indicate that the birthweight distribution is somewhat less polarized for girls (childsex $=2$ ) than for boys (childsex $=1$ ) and that this is due to a difference in distributional shape in the upper part of the distribution (overall relative polarization is driven by the URP). Option vce(svy) also works with variance estimation methods other than linearized (e.g. [Svy] brr), although in these cases one could also apply svy as a prefix command. ${ }^{20}$

\section{Acknowledgements}

I thank Eric Melse for valuable comments on earlier versions of the software that helped improving the command, Blaise Melly for a nudge on how to obtain the influence functions for quantiles of relative ranks, and Philippe Van Kerm for pointing me to the work by Joseph Gastwirth. Furthermore, I thank an anonymous reviewer for comments that helped improving the manuscript.

\section{References}

Abramson, I. S. 1982. On Bandwidth Variation in Kernel Estimates. A Square Root Law. The Annals of Statistics 10(4): 1217-1223.

Alderson, A. S., J. Beckfield, and F. Nielsen. 2005. Exactly How Has Income Inequality Changed?: Patterns of Distributional Change in Core Societies. International Journal of Comparative Sociology 46: 405-423.

Bernhardt, A., M. Morris, and M. S. Handcock. 1995. Women's Gains or Men's Losses? A Closer Look at the Shrinking Gender Gap in Earnings. American Journal of Sociology 101(2): 302-328.

\footnotetext{
${ }^{20} \mathrm{~A}$ fine distinction is that with vce (svy) the bandwidth for kernel density estimation (relevant for reldist pdf and reldist divergence with option pdf) will only be estimated once and then held constant across replications. With svy as prefix command, bandwidth estimation will be repeated in each replication.
} 
Bernhardt, A., M. Morris, M. S. Handcock, and M. A. Scott. 2001. Divergent Paths. Economic Mobility in the New American Labor Market. New York: Russel Sage Foundation.

Bliege Bird, R., D. W. Bird, B. F. Codding, C. H. Parker, and J. H. Jones. 2008. The "fire stick farming" hypothesis: Australian Aboriginal foraging strategies, biodiversity, and anthropogenic fire mosaics. Proceedings of the National Academy of Sciences 105(39): 14796-14801.

Botev, Z. I., J. F. Grotowski, and D. P. Kroese. 2010. Kernel density estimation via diffusion. Annals of Statistics 38(5): 2916-2957.

Chernozhukov, V., I. Fernández-Val, and B. Melly. 2013. Inference on Counterfactual Distributions. Econometrica 81(6): 2205-2268.

Clementi, F., V. Molini, and F. Schettino. 2018. All that Glitters is not Gold: Polarization Amid Poverty Reduction in Ghana. World Development 102: 275-291.

Cox, N. J. 2004. PPPLOT: Stata module for P-P plots. Statistical Software Components S438002, Boston College Department of Economics. Available from https: //ideas.repec.org/c/boc/bocode/s438002.html.

Ćwik, J., and J. Mielniczuk. 1989. Estimating density ratio with application to discriminant analysis. Communications in Statistics - Theory and Methods 18(8): 3057-3069.

. 1993. Data-dependent bandwidth choice for a grade density kernel estimate. Statistics \&s Probability Letters 16: 397-405.

Del Giudice, M. 2011. Sex Differences in Romantic Attachment: A Meta-Analysis. Personality and Social Psychology Bulletin 37(2): 193-214.

Deville, J.-C. 1999. Variance estimation for complex statistics and estimators: Linearization and residual techniques. Survey Methodology 25: 193-203.

DiNardo, J. E., N. Fortin, and T. Lemieux. 1996. Labour Market Institutions and the Distribution of Wages, 1973-1992: A Semiparametric Approach. Econometrica 64(5): 1001-1046.

Duncan, O. D., and B. Davis. 1953. An Alternative to Ecological Correlation. American Sociological Review 18(6): 665-666.

Eggers, A. C., and A. Spirling. 2016. arty Cohesion in Westminster Systems: Inducements, Replacement and Discipline in the House of Commons, 1836-1910. British Journal of Political Science 46(3): 567-589.

Firpo, S., N. M. Fortin, and T. Lemieux. 2009. Unconditional Quantile Regressions. Econometrica 77: 953-973. 
Gastwirth, J. L. 1975. Statistical measures of earnings differentials. The American Statistician 29(1): 32-35.

Hampel, F. R. 1974. The Influence Curve and Its Role in Robust Estimation. Journal of the American Statistical Association 69: 383-393.

Handcock, M. S. 2016. Relative Distribution Methods. Version 1.6-6. Available from https: //cran.r-project.org/web/packages/reldist/index.html.

Handcock, M. S., and E. M. Aldrich. 2002. Applying Relative Distribution Methods in R. University of Washington Working Paper No. 27. Available from http://dx.doi.org/ 10.2139/ssrn. 1515775 .

Handcock, M. S., and P. L. Janssen. 2002. Statistical Inference for the Relative Density. Sociological Methods and Research 30(3): 394-424.

Handcock, M. S., and M. Morris. 1998. Relative Distribution Methods. Sociological Methodology 28: 53-97.

—. 1999. Relative Distribution Methods in the Social Sciences. New York: Springer.

Hao, L., and D. Q. Naiman. 2010. Assessing Inequality. Thousand Oaks, CA: Sage.

Jann, B. 2004. DUNCAN: Stata module to calculate dissimilarity index. Statistical Software Components S447202. Available from https://ideas.repec.org/c/boc/bocode/ s447202.html.

- 2005. moremata: Stata module (Mata) to provide various functions. Statistical Software Components S455001. Available from http://ideas.repec.org/c/boc/bocode/ s455001.html.

. 2007. Univariate kernel density estimation. Available from https://doi.org/10. 7892 /boris. 69421.

. 2008. The Blinder-Oaxaca decomposition for linear regression models. The Stata Journal 8(4): 453-479. $708-737$.

. 2014. Plotting regression coefficients and other estimates. The Stata Journal 14(4):

. 2017. kmatch: Stata module for multivariate-distance and propensity-score matching, including entropy balancing, inverse probability weighting, (coarsened) exact matching, and regression adjustment. Statistical Software Components S458346. Available from https://ideas.repec.org/c/boc/bocode/s458346.html.

. 2020. Influence functions continued. A framework for estimating standard errors in reweighting, matching, and regression adjustment. University of Bern Social Sciences Working Papers 35. Available from https://ideas.repec.org/p/bss/wpaper/35.html. 
Le Breton, M., A. Michelangeli, and E. Peluso. 2012. A stochastic dominance approach to the measurement of discrimination. Journal of Economic Theory 147(4): 1342-1350.

Morris, M., A. D. Bernhardt, and M. S. Handcock. 1994. Economic Inequality: New Methods for New Trends. American Sociological Review 59(2): 205-219.

Panek, T., and J. Zwierzchowski. 2020. Median Relative Partial Income Polarization Indices: Investigating Economic Polarization in Poland During the Years 2005-2015. Social Indicators Research 149: 1025-1044.

Parzen, E. 2004. Quantile Probability and Statistical Data Modeling. Statistical Science 19(4): 652-662.

Reardon, S. F., and J. B. Townsend. 1999. SEG: Stata module to compute multiple-group diversity and segregation indices," , Boston College Department of Economics, revised 18 Apr 2018. Statistical Software Components S375001. Available from https://ideas . repec.org/c/boc/bocode/s375001.html.

Rios-Avila, F. 2020. Recentered influence functions (RIFs) in Stata: RIF regression and RIF decomposition. The Stata Journal 20(1): 51-94. 